\title{
Efficient and Adaptive Content Delivery of Linear and Interactive Branched Videos
}

\section{Vengatanathan Krishnamoorthi}

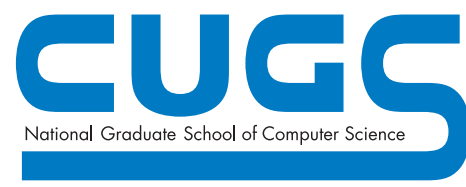

\footnotetext{
Department of Computer and Information Science Linköping University SE-581 83 Linköping, Sweden
} 
This is a Swedish Licentiate's Thesis

Swedish postgraduate education leads to a doctor's degree and/or a licentiate's degree.

A doctor's degree comprises 240 ECTS credits (4 year of full-time studies).

A licentiate's degree comprises 120 ECTS credits.

Copyright (C) 2016 Vengatanathan Krishnamoorthi

ISBN 978-91-7685-680-2

ISSN 0280-7971

Printed by LiU Tryck 2016

URL: http://urn.kb.se/resolve?urn=urn:nbn:se:liu:diva-131030 


\title{
Efficient and Adaptive Content Delivery of Linear and Interactive Branched Videos
}

\author{
by \\ Vengatanathan Krishnamoorthi
}

\begin{abstract}
Video streaming over the Internet has gained tremendous popularity over recent years and currently constitutes the majority of Internet traffic. The on-demand delivery of high quality video streaming has been enabled by a combination of consistent improvements in residential download speeds, HTTP-based Adaptive Streaming (HAS), extensive content caching, and the use of Content Distribution Networks (CDNs). However, as large-scale on-demand streaming is gaining popularity, several important questions and challenges remain unanswered, including determining how the infrastructure can best be leveraged to provide users with the best possible playback experience. In addition, it is important to develop new techniques and protocols that facilitate the next generation of streaming applications. Innovative services such as interactive branched streaming are gaining popularity and are expected to be the next big thing in on-demand entertainment.

The major contributions of this thesis are in the area of efficient content delivery of video streams using HAS. To address the two challenges above, the work utilizes a combination of different methods and tools, ranging from real-world measurements, characterization of system performance, proof-of-concept implementations, protocol optimization, and evaluation under realistic environments. First, through careful experiments, we evaluate the performance impact and interaction of HAS clients with proxy caches. Having studied the typical interactions between HAS clients and caches, we then design and evaluate content-aware policies to be used by the proxy caches, which parse the client requests and prefetch the chunks that are most likely to be requested next. In addition, we also design cooperative policies in which clients and proxies share information about the playback session. Our evaluations reveal that, in general, the bottleneck location and network conditions play central roles in which policy choices are most advantageous, and the location of the bottlenecks significantly impact the relative performance differences between policy classes. We also show that careful design and policy selection is important when trying to enhance HAS performance using proxy assistance.

Second, this thesis proposes, models, designs, and evaluates novel streaming applications such as interactive branched videos. In such videos, users can influence the content that is being shown to them. We design and evaluate careful prefetching policies that provides seamless playback even when the users defer their path choices to the last possible moment. We derive optimized prefetching policies using an optimization framework, design and implement effective buffer management techniques for seamless playback at branch points, and use parallel TCP connections to achieve efficient buffer workahead. Through performance evaluations, we show that our policies can effectively prefetch data of carefully adapted qualities along multiple alternative paths so to ensure seamless playback, offering users a pleasant viewing experience without playback interruptions.
\end{abstract}

This work has been supported by The National Computer Science Graduate School (CUGS).

Department of Computer and Information Science

Linköping University

SE-581 83 Linköping, Sweden 



\section{Acknowledgements}

This thesis would not have been possible without support and help from a number of people. First of all, I would like to thank my advisers Dr. Niklas Carlsson and Prof. Nahid Shahmehri for the opportunity, guidance and help in my graduate studies thus far. Working with you is very rewarding and makes for a great learning experience. Your dedication, attention to detail, and patience continues to inspire me, and I hope for more of it to rub off on me in the period to come.

I would also like to thank current and former colleagues in IDA for contributing to a very conducive work environment. Everyone in ADIT deserves a special mention, for being very accommodating and friendly. The lunch group at ADIT deserves a special shout out for having made my day on several occasions. Thank you all.

The administrative and technical support teams in IDA have been of immense help and support throughout my tenure here. Your presence has made handling administrative and technical matters hassle free.

I would also like to thank my family and friends for sharing several moments that I cherish deeply. Thank you for always being supportive and an endless source of fun. Last but not the least, I would also like to say thank you to my girlfriend Dharshini, for your endless love and patience. It means the world to me and I look forward to the future.

Thank you all! 



\section{Contents}

1 Introduction 1

1.1 Motivation and problem description ........... 3

1.2 Contributions . . . . . . . . . . . . . . 5

1.3 Thesis organization . . . . . . . . . . . . . 6

1.4 Publication list . . . . . . . . . . . . . 7

2 Background 9

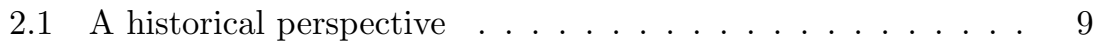

2.2 HTTP-based streaming . . . . . . . . . . . . . . . . 11

2.3 HTTP-based adaptive streaming . . . . . . . . . . . . . . 14

2.4 Web caching and caching of HTTP-based streams . . . . . 17

2.5 Other streaming techniques . . . . . . . . . . . . 19

2.6 Interactive branched video streaming, a primer . . . . . . . 21

3 Related work $\quad \mathbf{2 7}$

3.1 Content delivery .................... . . . 27

3.2 Client-driven approaches . . . . . . . . . . . . . . . . . . 29

3.2.1 Rate-adaptive streaming solutions before HAS . . . . 29

3.2.2 Client-driven approaches using HAS . . . . . . . . . 30

3.2.3 Interaction with TCP . . . . . . . . . . . . . . . . 32

3.2.4 Standardization efforts . . . . . . . . . . . . . 34

3.3 Proxy-driven approaches . . . . . . . . . . . . . . . . . . 35

3.4 Content personalization and interactive branched video . . . 37

4 Effects of proxy caches on HAS streams 41

4.1 Introduction . . . . . . . . . . . . . . . . . . . . . . . . 41

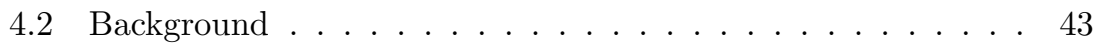

4.2.1 HTTP-based adaptive streaming . . . . . . . . 43 
4.2.2 SMP overview . . . . . . . . . . . . . . . 43

4.3 Methodology . . . . . . . . . . . . . . . 44

4.3.1 Experimental setup . . . . . . . . . . . . 45

4.3.2 Player instrumentation and metrics . . . . . . . . . . 45

4.3.3 Scenario-based evaluation . . . . . . . . . . . . 46

4.4 Player characterization . . . . . . . . . . . . . . . . . . . 48

4.5 Longitudinal proxy performance . . . . . . . . . . . 51

4.5.1 Baseline policies ............... 52

4.5.2 Quality and content-aware prefetching ....... 53

4.5.3 Client-proxy cooperation ............ 54

4.5.4 Performance evaluation ............. 55

4.6 Conclusion . . . . . . . . . . . . . . . 62

5 Prototype implementation of interactive branched video player over HAS $\quad 65$

5.1 Introduction . . . . . . . . . . . . . . . . . 65

5.1.1 Personalized interactive branched video . . . . . . 65

5.1.2 HTTP-based adaptive streaming . . . . . . . . . . 66

5.1.3 Design contributions . . . . . . . . . . . . 66

5.2 Interactive branched, nonlinear video . . . . . . . . . . . 68

5.3 System Design . . . . . . . . . . . . . . . . . . . . . 69

5.3.1 Design goals....................... 69

5.3 .2 System overview . . . . . . . . . . . . . . . . 70

5.3.3 Metafile structure . . . . . . . . . . . . 70

5.3.4 Longest-path matching. . . . . . . . . . . . 71

5.3.5 Prefetching and rate adaptation .......... . . 72

5.4 Implementation description . . . . . . . . . . . . . . 73

5.4.1 Implementation overview . . . . . . . . . . . 73

5.4 .2 Buffer management . . . . . . . . . . . . . 75

5.4 .3 Rate-adaptive prefetching . . . . . . . . . . 76

5.5 Proof-of-concept validation . . . . . . . . . . . . . 76

5.5.1 Experimental setup . . . . . . . . . . . 76

5.5 .2 Player validation . . . . . . . . . . 76

5.6 Conclusion . . . . . . . . . . . . . . . . 78

6 Prefetching strategies for interactive branched video stream$\begin{array}{lr}\text { ing over HAS } & \mathbf{7 9}\end{array}$

6.1 Introduction . . . . . . . . . . . . . . . . . 79

6.2 Personalized content delivery . . . . . . . . . . . . . 81

6.2.1 Chunks, segments, and branch points . . . . . . 81

6.2.2 Interactive branched, nonlinear media structure . . . . 82

6.3 Player management . . . . . . . . . . . . . . . . . . . 83

6.3.1 Problem description ............... 83

6.3.2 Objectives and constraints . . . . . . . . . . . 86

6.3.3 Prefetching policies. . . . . . . . . . . . . . . 88

6.3.4 Capped workahead . . . . . . . . . . . . 89 
6.4 Implementation description . . . . . . . . . . . . . . . 90

6.4 .1 Software overview . . . . . . . . . . . . . . . . 90 90

6.4.2 High-level system overview . . . . . . . . . . . . . 90

6.4.3 Interactive playback . . . . . . . . . . . . . . . . 91

6.4.4 Parallel connections . . . . . . . . . . . . . . . . . . 91

6.4.5 Rate estimation . . . . . . . . . . . . . . . 92

6.5 Experimental results . . . . . . . . . . . . . . . . . 92 92

6.5.1 Experimental setup . . . . . . . . . . . . . . . . 92 92

6.5.2 Single-branch experiments . . . . . . . . . . . . . . 93

6.5.3 Multiple branch point scenarios . . . . . . . . . . . . 95

6.5.4 Competing flows . . . . . . . . . . . . . . . 96

6.5.5 Capped workahead . . . . . . . . . . . . . 97

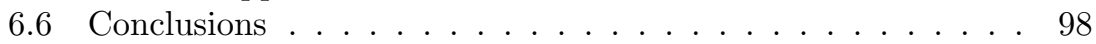

7 Conclusion and future work 101

7.1 Future work . . . . . . . . . . . . . . . . . 102 



\section{List of Figures}

2.1 Generating chunks from a base video . . . . . . . . . . . . 12

2.2 Typical download pattern of HTTP-based players . . . . . . . 13

2.3 Multiple chunk encoding-rates in a HAS video . . . . . . . . . 15

2.4 Example linear video organized into segments for interactive branched playback . . . . . . . . . . . . . . 22

2.5 Interactive branched playback $\ldots \ldots \ldots \ldots \ldots$

4.1 Synthetic baseline traces for the available bandwidth . . . . . 47

4.2 Real-world traces of available bandwidth . . . . . . . . . 48

4.3 Player performance comparison of SMP 1.6 and 2.0 under the three synthetic baseline scenarios. . . . . . . . . . . . . . 49

4.4 Performance impact of buffer sizes using the fast varying synthetic bandwidth trace. . . . . . . . . . . . . . 50

4.5 Video quality under real-world scenarios for different buffer sizes. . . . . . . . . . . . . . . . . . . 51

4.6 Stall times and startup delays under real-world scenarios for different buffer sizes. . . . . . . . . . . . . . . . . 52

4.7 Comparison between baseline and content-aware proxy policies, and the bottleneck is between the clients and proxy. . . 57

4.8 Observed quality levels over $n$ subsequent client downloads when using 1-ahead prefetching with client-proxy bottleneck. $\quad 57$

4.9 Hit rate as a function of the number of previous downloads, when either client-proxy or proxy-server bottleneck. . . . . . 57

4.10 Comparison between baseline and content-aware policies; proxyserver bottleneck. . . . . . . . . . . . . . . . . . 59

4.11 Client-proxy cooperation experiments, when client-proxy and proxy-server bottleneck. . . . . . . . . . . . . . . . . 61 
4.12 Observed quality levels over $n$ subsequent client downloads when the cooperative buffer oblivious policy is used, and the bottleneck is between the proxy and server. . . . . . . . . . 61

4.13 Summary of quality level statistics under different policies when using larger client buffer. . . . . . . . . . . . . . . 62

4.14 Summary of stall times under different policies when using larger client buffer. . . . . . . . . . . . . . 63

5.1 Example media structure. . . . . . . . . . . . . . 68

5.2 High-level design and buffer management. . . . . . . . . . 72

5.3 Example structure of an interactive branched video. . . . . . 73

5.4 Timestamps when an example client request and download different segments. . . . . . . . . . . . . . . . . . . . . . 74

5.5 Playback quality. . . . . . . . . . . . . . . 77

6.1 Illustration of terminology. . . . . . . . . . . . . . . 82

6.2 Round-robin parallel downloading . . . . . . . . . . . 85

6.3 Playback qualities in default scenario. . . . . . . . . . . . . . 94

6.4 Impact of the available bandwidth. . . . . . . . . . . . . . . . 95

6.5 The average playback rate under different end-to-end RTTs, number of chunks $n_{e}$ in the initial segment, and the number of branch options $\left|\mathcal{E}^{b}\right| \ldots \ldots \ldots$. . . . . . . . . . . . 96

6.6 The stall probability under different end-to-end RTTs, number of chunks $n_{e}$ in the initial segment, and the number of branch options $\left|\mathcal{E}^{b}\right| . \ldots \ldots$. . . . . . . . . . . . . . 97

6.7 Average playback rate in scenario with multiple branch points, under different conditions. . . . . . . . . . . . . . . . . 98

6.8 Stall probability at the second branch point under different conditions. . . . . . . . . . . . . . . . . . . 99

6.9 The average video playback rate and stall events for different number of competing flows. . . . . . . . . . . . . . . . . . . . 99

6.10 Buffer occupancy over time . . . . . . . . . . . . . 100 


\section{List of Tables}

4.1 Bandwidth usage between proxy and server for the policies defined in Sections 4.5.1 and 4.5.2. . . . . . . . . . . . . 58

4.2 Average playback quality (measured in Kbps) of example policies under different scenarios. . . . . . . . . . . 60

5.1 Stall events and branch times. . . . . . . . . . . . . 77

6.1 Notation for interactive branched nonlinear media . . . . . . 84

6.2 Summary of prefetching policies. . . . . . . . . . 89 



\section{Chapter 1}

\section{Introduction}

The World-Wide Web (WWW) began as a system to organize and retrieve information over the Internet, based on pages containing hypertext. Both the WWW and the Internet have continually undergone changes and have now transformed into a distribution system for hypermedia, which in addition to hypertexts also includes graphics, audio, video, and plain text. Transformations of the WWW has facilitated deployment of more and ever improved web-based services. Today's network applications and services have come to dominate several aspects of our day-to-day lives, including education, business, banking, communication, and entertainment.

In contrast to early services such as email, Internet Relay Chat (IRC), telnet, File Transfer Protocol (FTP), and WWW, which have been available for commercial use in different embodiments since the 1980s, video streaming did not appear as a commercial service until the late 1990's. Video streaming, a technique that allows clients to start playback of a video before having downloaded the entire file, provides a valuable service to many users. These services were initially difficult to realize, since many networks did not have the capacity to satisfy the high bandwidth requirements of video. Deployment of such services were also hampered by a lack of efficient delivery architectures, video-encoding, compression, and distribution techniques.

By the time the first well known commercial streaming video players appeared (e.g., RealNetwork's RealPlayer ${ }^{1}$, Microsoft's ActiveMovie ${ }^{2}$ and Apple's QuickTime ${ }^{3}$ player), residential Internet speeds were for the first time sufficient to stream a low-quality video without stalls, although first adopters of dial-up Internet services might remember otherwise. Since then much has happened, and today video and audio entertainment services are

\footnotetext{
${ }^{1}$ https://en.wikipedia.org/wiki/RealPlayer

${ }^{2}$ https://en.wikipedia.org/wiki/ActiveMovie

${ }^{3}$ https://en.wikipedia.org/wiki/QuickTime
} 
being delivered to the masses over the Internet. The feasibility and common usage of these services can be attributed to the recent improvements in network bandwidths, the computational power available at end hosts, and the adoption of scalable content-delivery techniques. These improvements not only make efficient distribution of high volumes of data possible, but also open gateways to new and innovative services.

On-demand streaming, as exemplified by services such as YouTube and Netflix, have become the largest source of traffic on the Internet today. A recent study by Sandvine [1] suggests that $60 \%$ of all traffic over the Internet in North America can be attributed to video on-demand streaming. A significant share of this traffic is from websites such as Netflix (31\%) and YouTube $(12.2 \%)$. Similar trends have also been observed in other continents. Aside from on-demand streaming, live-streaming services (that streams live events over the Internet), has also gained tremendous popularity. More and more mainstream events such as the Olympics, FIFA World Cup, and several other sport events, news and other mass media are moving towards disseminating content over the Internet. Several national and regional TV channels, such as BBC in the United Kingdom and SVT in Sweden have their shows available both live and on-demand for clients within their respective countries ${ }^{4,5}$.

Recent advances in on-demand streaming techniques have also made interactive on-demand streaming possible. These services, in addition to being on-demand, allow the viewer to interact with the video based on predefined or dynamic personalization options. This includes videos that provide users with a 360-degree field-of-view or interactive plot-lines where the user chooses the outcome of certain scenes. Across such services and scenarios, it is paramount that the user interaction and the resulting change in the streaming video playback is seamless to the user, as re-buffering events can be very frustrating to a viewer. Such applications are only recently beginning to emerge but have generated significant interest from the mass-media and from the user communities. For example, websites such as Interlude ${ }^{6}$ and others similar to it, allow users to create interactive storylines using a web-based editor. Here, certain objects of a base video can be annotated with clickable interfaces or any other way of reading user input. The viewer, when watching a video, can then click on these objects and personalize the plot to their liking while viewing the video. Interactive videos based on annotations are also common in several YouTube channels, where the viewer can click on an annotation to go to a different video, although this process is not seamless. Similar extensions can also be derived using virtual-reality headsets such as the Google Cardboard ${ }^{7}$ or the Oculus Rift ${ }^{8}$ where the user is only shown a portion of the viewable content, and the user interaction

\footnotetext{
${ }^{4}$ http://www.bbc.co.uk/iplayer

${ }^{5}$ http://www.svtplay.se

${ }^{6}$ https://interlude.fm/

${ }^{7}$ https://www.google.com/get/cardboard/

${ }^{8}$ https://www.oculus.com/en-us/rift/
} 
by means of looking or gazing at an object or interacting with a hand-held device can be used to drive interactive videos.

The sheer volume of videos and users of these services, their wide-spread popularity, and the increasing user base of on-demand streaming [2] require current and future video streaming content delivery systems to be highly scalable and efficient. Such requirements have lead to the deployment of purpose built Content Distribution Networks (CDN) for video streaming and deployment of caches at the edges of access networks, allowing popular content to be delivered from closer to the user. In addition to the challenges posed by on-demand streaming, emerging services such as interactive streaming pose unique challenges where user satisfaction is strongly coupled to the service being seamless and of high quality. Although residential Internet speeds have considerably increased, improved downloading and prefetching strategies are required to provide an acceptable Quality of Experience (QoE) to the viewer, while at the same time scale to a large number of users in a cost effective manner. Asides from the technical factors mentioned above, socio-economic and human factors play important roles in the revenue model of such services. The combination of all these factors makes for a very interesting field of research.

\subsection{Motivation and problem description}

The average residential bandwidth has steadily increased in the past few decades and conservative predictions are that residential bandwidths would double in the period between 2014 and 2019 [3]. Studies have also shown that high-end residential data rates grow by as much as $50 \%$ annually [4]. The highly competitive nature of residential Internet access ensures that these advances trickle down very quickly to the average user. Together with the much increased residential bandwidths, several high-quality on-demand video streaming services have emerged. Typically, in these services, the viewer can chose from a catalog of videos and the video stream is served to a client only when explicit requests for the video are made. Such services break the barriers imposed by traditional broadcast services, where the viewer had to tune in to a channel at a particular time.

Several methods have been explored to deliver video streams over the Internet. Currently, the most popular means is through HTTP-based Adaptive Streaming (HAS) ${ }^{9}$. This family of streaming protocols utilizes the HTTP protocol, originally designed for the web, together with adaptive quality selection to match the client's current download rate. Analogous to the web, streaming through HAS is also client-driven and takes added benefits of components developed and deployed for the web. HAS content can easily

\footnotetext{
${ }^{9}$ We will use the acronym HAS to refer to all HTTP-based Adaptive Streaming (HAS) solutions, including proprietary players such as YouTube, Netflix, Amazon Prime, etc., and new standards such as Dynamic Adaptive Streaming over HTTP (DASH) [5]
} 
be replicated, stored and delivered by caches and off-the-shelf HTTP servers, without the need for any specialized or proprietary software.

Given that almost all commercial services have shifted towards using HAS for video streaming, continued improvements in scalability and efficiency are required to cater to the ever increasing user base. The increasingly common trend of television viewers resorting to on-demand, IP-based sources for their entertainment is expected to trigger several changes to the traffic on the Internet. Predictions by Cisco [3] suggest that more and more traffic on the Internet will originate from CDNs and that the global IP video traffic will account for $80 \%$ of all IP traffic on the Internet. It is therefore important that both current and next generation of streaming services are scalable and efficient. Content caching and CDNs provide valuable tools here. However, while CDNs and content caches are easy to implement with HAS-based content, their impact on streaming performance are relatively unexplored.

Furthermore, the ever changing landscape of Internet-based services has recently transitioned into a new phase, where personalized on-demand services are replacing the traditional broadcast-based entertainment services. As this significant change in user behavior continues, we will see many new entertainment services that require new delivery solutions to be developed. In the context of these next-generation services, there are many interesting and important research questions pertaining to problems related to content organization, optimized client-side implementations, efficient content delivery, and content caching that remain largely unexplored.

This thesis contributes towards (i) improving the current state-of-theart for efficient delivery of regular linear HAS videos (where viewers watch a video from start to end, and the only allowed actions are play, pause and fast-forward/backward) with the help of caches, and (ii) to enable tomorrows' personalized services with the introduction of new HAS-based interactive streaming protocols that allow users to select their own plot sequences through a video. Both these aspects are important to realize several of the aforementioned projections. First, we study the effect of proxy caches on streaming over HTTP. We propose and evaluate new policies for proxy caches which leads to a better user experience and better bandwidth utilization. Our contributions help to bridge the gap in understanding the effect of proxy caches on HAS and answers important questions as to how content-aware proxies must be built. Second, we propose, implement, and evaluate a framework for interactive branched video streaming over HTTP. By leveraging HTTP, our framework allows a content-creator to personalize a video to users' tastes, and seamless user interaction with the video. Asides from developing such a framework, we address several research questions related to design of interactive branched video players, design and evaluation of classes of prefetching policies and buffer management techniques, that are required to provide stall free playback of such videos. In general, our research methodology relies on thorough evaluation and characterization of 
both existing and our proposed solutions through system implementations and real-world experiments. Whenever possible, our source codes have also been released for use by the research community.

\subsection{Contributions}

This thesis contributes efficient and adaptive techniques to deliver on-demand streaming of both linear, non-interactive video and interactive branched video. This thesis makes four primary contributions.

(a) Performance study of the impact that web proxy caches have on HTTP-based Adaptive Streaming (HAS). Caching of video content and subsequent playback of cached content by other clients is one of the biggest motivations for streaming over HTTP. Although HAS can leverage potential benefits of caches, the interaction between HAS clients and proxy caches is relatively unexplored and requires deeper investigation. Analysis of the impact of proxy caches on HAS was performed by first instrumenting a HAS player and by creating a dedicated testbed that included a Squid proxy and a measurement framework to collect and analyze network traffic. We then used the testbed to run trace-driven network emulations for a wide range of scenarios, in which we collect traces, both at the network-level and at the instrumented player, and analyze the results. Our results show that a standard proxy can assist HAS clients, but that the location of the bottleneck and network conditions play important roles in a proxyassisted HAS scenario. Our results also show that the effectiveness of proxy caches can be vastly improved by utilizing content-aware proxy caches.

(b) Propose and evaluate new policies for proxy caches that improve user experience, scalability, and efficiency of HAS. Our content-aware proxy cache policies keep track of how HAS videos are chunked and utilize that on-demand videos typically are viewed sequentially from start to end. These prefetching policies are placed at the proxies and keep track of a HAS client's progress through a video, and prefetches chunks at the quality that is most likely to be requested by the client. All these policies were implemented in an open source Squid proxy. In addition to content-aware policies, we also design and evaluate scenarios where a HAS client and the proxy share information, such as the current buffer condition and chunks available in the cache, respectively. With the help of this additional information, both the client and the proxy can make informed decisions on which chunks to download and their respective qualities. Our evaluations show that the content-aware proxy policies and the cooperative proxy assisted solution provide improved playback quality and 
cost savings when compared to a simple proxy-assisted solution. As with the proxy-assisted solution, the effectiveness depends upon the location of the bottleneck and the available bandwidth on each side of the proxy.

(c) Propose, implement, and evaluate novel interactive VoD delivery techniques over HAS that allows interactive branched video playback. Interactive branched video, sometimes also referred to as nonlinear video or multipath video, is an extension to on-demand HAS, where the viewer can chose to view one of many plot sequences in a video. These plot sequences can be defined by the creator and can be modified online based on user information such as previous viewing history or user profile. This thesis presents the design and evaluation of a framework that allows interactive branched video playback over existing HAS infrastructure with modifications only to the client-side player. Using this framework, seamless transition across branches with adaptive playback quality can be achieved by leveraging the chunked nature of HAS videos, even with simple prefetching techniques.

(d) Design an optimization framework for interactive branched video, classes of prefetching policies to provide stall free and optimal playback, and the evaluation thereof. Interactive branched video playback over HAS allows for interactive and adaptive story telling, while at the same time careful prefetching and buffer management policies are required to achieve uninterrupted playback. This thesis presents the design, implementation and evaluation of prefetching and buffer management policies, which are developed based on an optimization framework. Results show that under a wide range of scenarios, our policies can effectively prefetch data by using multiple TCP connections and choosing qualities for chunks such that the playback is stall free, seamless, and the playback quality is maximized.

\subsection{Thesis organization}

This thesis is organized around the main contributions as described in Section 1.2. To familiarize the reader, background (Chapter 2) and related works (Chapter 3) are first presented. Chapter 4 presents the evaluation and characterization of a typical HAS video player, followed by our evaluation of how the presence of proxy caches affects the performance of such players. Chapter 4 also discusses the design and evaluation of various proxyassisted streaming techniques and policies that could be used to mitigate the potential negative performance effects of a proxy cache, while at the same time retaining the improvements to efficiency and scalability that a proxy cache adds. In Chapter 5, we present the motivation and design issues posed by interactive branched video streaming, followed by a discussion and 
evaluation of a simple prototype implementation of an HTTP-based interactive branched video player. Chapter 6 presents a detailed formulation, implementation, and evaluation of the prefetching strategies and solutions required for interactive branched streaming over HTTP. Finally, concluding remarks, extensions of the work presented in this thesis, and future works are discussed in Chapter 7.

\subsection{Publication list}

This thesis is based three research papers [6], [7], [8]. In the following we summarize these papers, as well as another paper under submission [9] and others previously published by the defendant [10], [11].

\section{Articles in thesis:}

- V. Krishnamoorthi, N. Carlsson, D. Eager, A. Mahanti, and N. Shahmehri, Quality-adaptive Prefetching for Interactive Branched Video using HTTP-based Adaptive Streaming In Proceedings of the ACM International Conference on Multimedia (ACM Multimedia), Nov. 2014.

- V. Krishnamoorthi, N. Carlsson, D. Eager, A. Mahanti, and N. Shahmehri, Helping Hand or Hidden Hurdle: Proxy-assisted HTTPbased Adaptive Streaming Performance. In Proceedings of the IEEE International Symposium on Modeling, Analysis and Simulation of Computer and Telecommunication Systems (IEEE MASCOTS), Aug. 2013.

- V. Krishnamoorthi, P. Bergström, N. Carlsson, D. Eager, A. Mahanti, and N. Shahmehri, Empowering the Creative User: Personalized HTTP-based Adaptive Streaming of Multi-path Nonlinear Video, In Proceedings of the ACM SIGCOMM Workshop on Future Human-Centric Multimedia Networking (FhMN), Aug. 2013. This paper also appeared in ACM SIGCOMM Computer Communication Review (CCR), Oct. 2013.

Other research articles:

- V. Krishnamoorthi, N. Carlsson, D. Eager, A. Mahanti, and N. Shahmehri, Bandwidth-aware Prefetching for Proactive Multi-video Preloading and Improved HAS Performance. In Proceedings of the ACM International Conference on Multimedia (ACM Multimedia), Oct. 2015.

- N. Carlsson and D. Eager and V. Krishnamoorthi and T. Polishchuk and N. Shahmehri, Optimized adaptive streaming of multi-video stream bundles, Under submission. 
- A. Bianco and V. Krishnamoorthi and L. Nanfang and L. Giraudo, OpenFlow driven ethernet traffic analysis, In Proceedings of the IEEE International Conference on Communications (IEEE ICC), June 2014. 


\section{Chapter 2}

\section{Background}

\subsection{A historical perspective}

The requirements of on-demand streaming are quite different than those of traditional web-based services. In on-demand streaming, the time between a certain piece of a video beging downloaded, and until the same piece is played back is typically quite small, and is bounded by the client's playback buffer. In typical scenarios, where clients often want small startup delays (the time between the user starting playback of a video to when the video actually commences playback), this constraint leaves very little time for the transport layer to perform error correction and recovery tasks. As the the first commercial streaming services emerged, the networks had larger round-trip times and lower datarates in average, when compared to what we see today. These limitations played significant roles when design choices regarding the transport protocol were made.

Most of the original video streaming services at the time were designed with the User Datagram Protocol (UDP) in mind, rather than with the help of the Transmission Control Protocol (TCP). UDP offers no guarantees, but allows the sender to control the sending rate. In contrast, TCP offers reliable connection-oriented services with congestion and flow control, but does not provide any rate-control. To ensure timely delivery, it was therefore often argued that a client would prefer to have a few packets lost, rather than experience additional delay for packet-level error correction or recovery mechanisms to correct the stream. To overcome errors that might occur due to UDP's properties, it was common that a certain level of robustness was built into the encoded video, allowing the player to recover from such situations without additional retransmissions.

At that time, several proprietary application and transport layer protocols were also developed to manage streaming sessions. The first among 
these were the Progressive Networks Media/Audio (PNM/PNA) protocol developed by RealNetworks. They, as well as many others, later moved to using a proprietary transport protocol over the Real Time Streaming Protocol (RTSP) [12] to manage video streaming sessions. The RTSP protocol was developed and standardized by the Internet Engineering Task Force (IETF) and is also known as the network remote control. The protocol places no restrictions on the transport layer to be used. Several commercial solutions have utilized RTSP with either TCP, UDP, and even proprietary transport protocols. Microsoft has developed multiple standards based both on RTSP and on their own standard called Microsoft Media Server (MMS) ${ }^{1}$, which supports transfers over both TCP and UDP. Adobe, on the other hand, developed the Real Time Messaging Protocol (RTMP) [13] which supports transfer over multiple transport layer protocols. Standardization attempts were also made at the transport layer, which lead to the Real Time Protocol/Real Time Control Protocol (RTP/RTCP) [14] suite, which was built on top of UDP. These example protocols also illustrate that there has long been a very large variety of protocols that could be used to stream videos.

Although arguments about TCP being unsuitable for streaming traffic may have held some water during the late 90s and early 2000s, the use of proprietary or UDP-based protocols had major limitations. Perhaps the biggest among these was that traffic over UDP or other unstandardized protocols often are blocked by firewalls and Network Address Translators (NATs), due to security concerns with connectionless service such as UDP and due to risks posed by unknown protocols, for example. Furthermore, several protocols such as RTP/RTCP utilized in-band or out-of-band signaling through specialized protocols, which were even used to drive feedback-based quality adaptation at the servers. This meant that the servers had to track the client state continually, imposing greater requirements at the server-side as well. These limitations slowly started to outweigh the benefits of using UDP-like protocols.

The WWW, during the same period, had grown tremendously. Service and content providers had developed and deployed technologies to scale up to increasingly larger user bases. Several of the largest websites and other companies built CDNs, consisting of vast interconnected server networks which can be used to deliver content globally. Also, web caches were deployed, which store a copy of webpages that flow through it. These caches copy locally the webpages, allowing for significant reduction in fetch times of later requests to the same webpages. Both $\mathrm{CDNs}$ and caches were developed specifically for web-based traffic. The WWW uses the Hypertext Transfer Protocol (HTTP) as its application layer protocol and the TCP/IP protocol suite to provide guarantees that are required for webpages. The development and deployment of CDNs and proxy caches significantly reduced the cost incurred by the content provider and the service providers in delivering data to an end user.

\footnotetext{
${ }^{1}$ https://en.wikipedia.org/wiki/Microsoft_Media_Server
} 
At the same time, incremental improvements to access speeds and reduction in Round-Trip Times (RTTs) made streaming over TCP a feasible alternative. Furthermore, as client side computational power and storage capacity improved, the clients could use a larger buffer to accommodate for fluctuations in the network bandwidth. The larger buffer also provides additional time for TCP's error correction and recovery to recover packets in time for playback. These developments, in addition to improvements in delivering web content through CDNs, large deployments of proxy caches at the network's edge and NAT/firewall ports not blocking client-driven TCP traffic lead to the adoption of streaming over HTTP. In contrast to the earlier streaming protocols, HTTP and therefore streaming over HTTP is entirely client driven. This significantly reduces the complexity required at the server-side, helps the entire system to scale better, for e.g., by making use of CDNs and proxy caches and even allows the possibility to download streams from multiple servers in parallel. Finally, deployment and licensing costs of HTTP servers are much lower when compared to deployment of proprietary servers.

\section{$2.2 \quad$ HTTP-based streaming}

HTTP is the application level protocol of the web, and, as one would expect, it is designed with a client-server model in mind. HTTP, as mentioned before, is client driven. A client can establish a HTTP session by connecting to port 80 on a server through a TCP connection. Having established a connection, clients can then request or transfer data from the server using standard HTTP methods. The most popular among such methods is the GET method, used to retrieve content, and POST to transfer data to the server. Along with responses, the server can also send HTTP response codes, which are used to convey information regarding progress of a request, success or completion of a request, as well as regarding redirections and errors.

HTTP was originally designed to transfer webpages and small objects, such as images and animations, that might be embedded on webpages. On the other hand, videos of even a few seconds in duration are considerably larger than an average webpage. However, the first generation of HTTPbased streaming players requested for the entire video using a single GET request. The rate at which the server transmits the request is governed by TCP's congestion control algorithm, with the TCP/IP stack delivering data on a best-effort basis. Therefore, the rate at which a client receives the video stream is dependent on the available bandwidth between the two hosts, the rate at which the server can send data, and the rate at which the client can receive data. HTTP requests which follow this method to download are known as progressive downloads.

Although progressive downloads work well for short video clips where the viewer watches from the beginning of a video to its end, there are several issues when one takes into account a typical user's behavior. For example, 


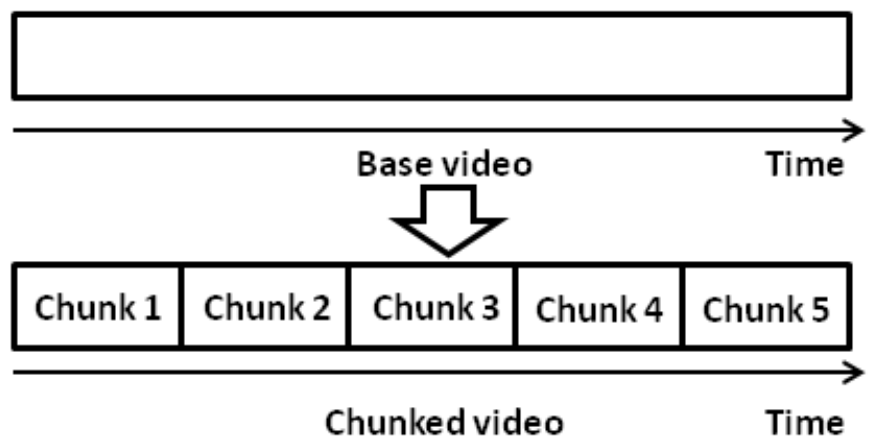

Figure 2.1: Generating chunks from a base video

studies have shown that users seldom watch videos from start to end [15]. In fact, viewers often navigate to parts of the video which they consider interesting or even worse, watch the beginning of several videos before settling down to watch a video completely [16]. Under such use cases, a client which progressively downloads a video stream would have to wait for a long time if the viewer decides to seek to the end of the video as soon as playback commences. Furthermore, TCP's congestion control is the only factor which throttles the download rate in progressive HTTP download. In cases where a viewer decides to abandon watching a particular video, there is no benefit to download data which lies a few seconds beyond the current play-point. In both the aforementioned cases, a large portion of the download data might not have been used for playback. This leads to wasted bandwidth at both the client and server side, potentially forcing the content provider to deploy additional server replicas to meet the bandwidth demands.

To overcome the apparent shortcomings of progressive download video streams, commercial solutions use either a chunk-based or range-requestbased system. Both these systems are quite similar, but differ slightly only in the way in which the video is represented in the server.

A chunk-based system divides a base video into smaller pieces called chunks, as shown in Figure 2.1. Successive chunks are continuations of the original video byte stream. Placement of the boundary between chunks is not based on data volume, but on the playtime of each chunk. The chunk duration is the number of seconds that every chunk in that video will play for. Typical chunk lengths that one might observe are 3-10 seconds. These values vary between different services and even between two videos in a service. Once chunks are generated, each chunk is assigned a unique Uniform Resource Locator (URL). Generally, the assigned URLs are based on the URL which identifies the video, and a number is typically added as a suffix to indicate the relative chunk position of the chunk.

In contrast, a range-request based system does not partition the byte 


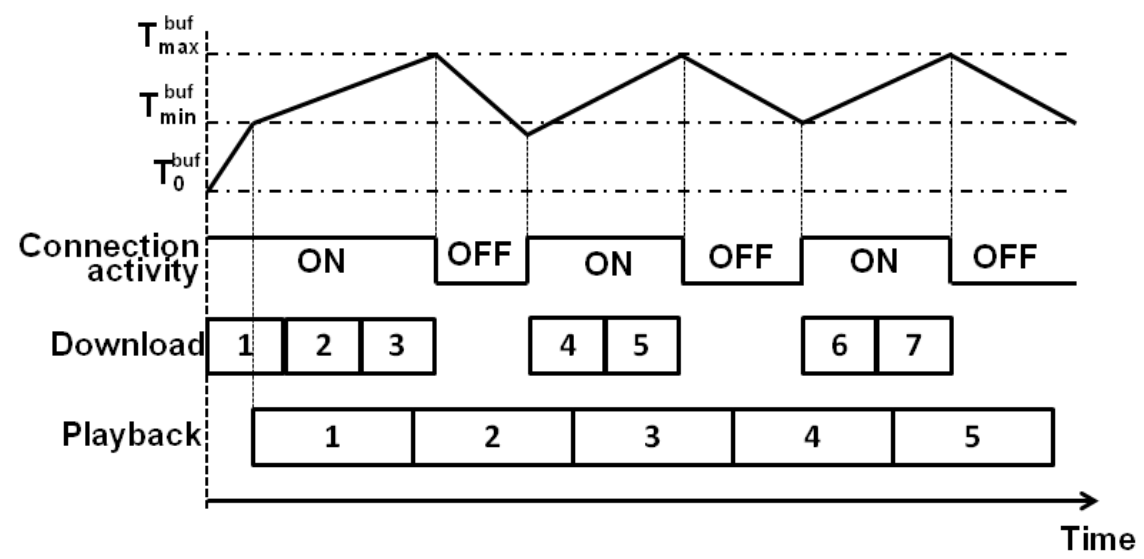

Figure 2.2: Typical download pattern of HTTP-based players

stream into chunks, but instead, relies on HTTP range-requests. With such requests, the client can independently request any sequence of bytes in the video stream by using a start and an end byte value. One can imagine the progressive download as a HTTP range-request with the start value as zero and the end value as the final byte of the video.

In contrast to progressive download, both the chunk-based and rangerequest-based systems require the client to have some additional information about the video. In a chunk-based system, the client must know the chunk length, the number of chunks available, and the naming convention used for that video. Similarly, a range-request-based client must know the first and the last byte of a video stream and the protocol stack must support HTTP range-requests, otherwise known as byte serving. With these systems, a manifest file, also called as the Media Presentation Description (MPD), is used to share all relevant information to the client. Whenever playback of a video is initiated, the manifest file is sent to the client along with player binaries and it provides all bootstrap information necessary at the client. The data contained in the manifest can also be used to map a certain playtime to a specific chunk or range-request.

In special cases, such as interactive or multi view-point streaming, MPDs can also be used to convey information about interaction points in the video, or multiple viewing angles. There might also be additional configuration information about the adaptation logic and codec related information in the file. Once the MPD has been parsed, the client can be expected to act in accordance to the conventions of the service and start downloading chunks for playback.

Using chunks or range-requests overcomes the aforementioned drawbacks of progressive downloads. When a viewer decides to seek to new point in time, the player can now request for a chunk or range of bytes that corre- 
spond to play-time requested by the viewer. This eliminates the unnecessary downloading of data between the current play-point and the new play-point. With chunk and range-request-based players, maximum buffer sizes can now be controlled, as the request for each chunk or byte-range can be used to control how much future data is available in the buffer. The maximum buffer size $\left(T_{\max }^{b u f}\right)$, can be defined as the maximum number of seconds of video that the client can locally store in its buffer. Once $T_{m a x}^{b u f}$ is reached, the player can be instructed to not request for future chunks or ranges. By using another threshold, called minimum buffer size $\left(T_{\text {min }}^{b u f}\right)$, which corresponds to the minimum value that the buffer should ever reach, the buffer can be allowed to drop until $T_{\text {min }}^{b u f}$ is reached, before requests are triggered again. Using this simple technique, buffer sizes can be regulated to always remain between these two thresholds. The size and difference between these two values must be carefully considered to allow for stall free playback, minimal bandwidth and resources wastage as well.

Controlling the amount of data available in the buffer through thresholds, in steady-state results in two distinct phases of operation. Whenever the buffer occupancy exceeds $T_{\text {max }}^{b u f}$, the client does not place any requests until the buffer occupancy drops to $T_{\min }^{b u f}$. Since $T_{\max }^{b u f}$ and $T_{\min }^{b u f}$ are actually set in terms of play-time (seconds) the player remains in this state for the duration given by $\left(T_{m a x}^{b u f}-T_{m i n}^{b u f}\right)$. Similarly, the client would download chunks whenever the buffer has fallen below $T_{\text {min }}^{\text {buf }}$ and has not yet reached $T_{\text {max }}^{b u f}$. The duration that a client would remain in this state will be equal to $T_{\max }^{b u f}-T_{\min }^{\text {buf }} *($ encodingrate/downloadrate). In popular literature, these two states are often called the off and on states, respectively. Figure 2.2 shows a diagrammatic explanation of these two states with the associated buffer occupancy, as well as the download and playback details for a chunk-based system.

\section{$2.3 \quad$ HTTP-based adaptive streaming}

HTTP-based Adaptive Streaming (HAS) is a widely used standard to deliver video streams over HTTP. HAS is very similar to the chunk and range-based streaming over HTTP. In fact, both the download strategies and the way in which chunks/ranges are downloaded are exactly the same. However, in contrast to HTTP-based streaming, a HAS stream has multiple encodings of the same video available on the server. Furthermore, the client when watching the video, is free to adaptively switch between different qualities of the video and audio chunks (from the set of qualities available) as it is progressing from one chunk to the next. Figure 2.3 shows a representation of multiple encoding rates of a base-video organized into chunks whose boundaries align perfectly.

Generally video chunks or range-requests are generated in a way such 


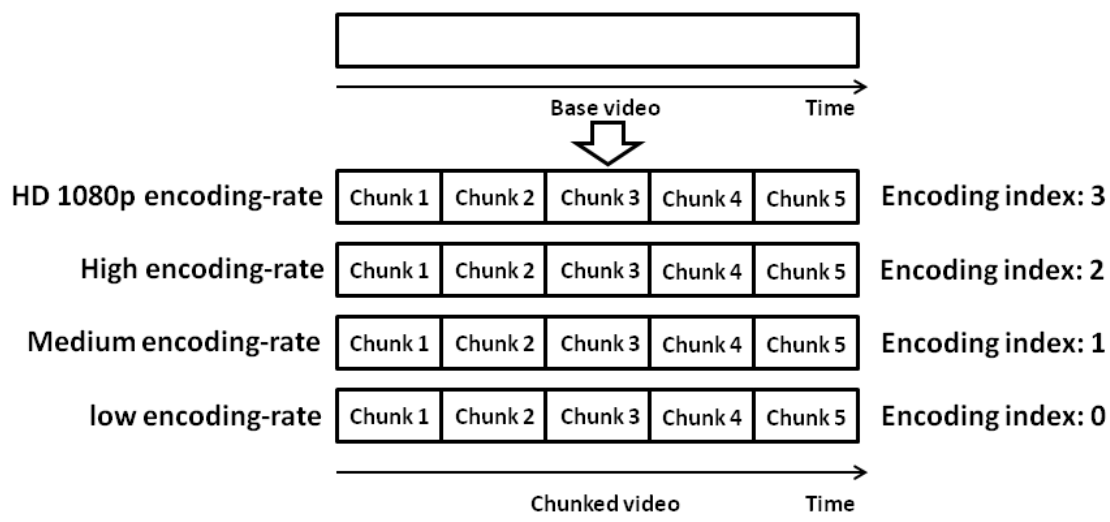

Figure 2.3: Multiple chunk encoding-rates in a HAS video

that the beginning of each chunk or range request aligns with an I-frame. Here, I-frame stands for Intra-coded frame. These frames are fully specified; i.e., the decoder can reconstruct this frame on the screen without any additional information. Since I-frames contain all information about a scene without dependencies to earlier or later frames, they are considerably larger compared to P-frames (Predicted frame) and B-frames (Bi-predictive frame) which can refer to frames before and after it.

Some chunk or range-request based HTTP-based players allow the clients to manually chose an encoding based on their expected needs. This is not HAS. In true HAS videos, the chunks are synchronized across encoding-rates (e.g., as shows in Figure 2.3) so that, the start and end time of chunks are exactly the same across all available encoding-rates the video is available at. This allows the client to run rate-estimation algorithms to determine the best quality at which the next chunk should be requested. The qualities are selected in such a way that the clients are expected to achieve high playback quality, while avoiding playback stalls.

Quality adaptation algorithms are used in HAS to determine the quality at which the next chunk is downloaded. Since chunk boundaries are aligned across all encodings of the video, the client, if necessary, can chose to playback chunks at any quality, irrespective of the qualities choice before. In order to ascertain the network throughput, a HAS player tracks the time at which requests are sent to the server $\left(T_{\text {request }}\right)$. The client is aware of the sizes $(S)$ of these chunks based on the information available in the manifest file, or in the case of range-requests the size of the range-request is determined by the player. When a chunk has been downloaded completely (indicated by a 200 OK HTTP response code), the client also tracks the time at which the response was received $\left(T_{\text {response }}\right)$. Using these parameters, the client can now generate the average download rate at which this chunk was downloaded as $S /\left(T_{\text {response }}-T_{\text {request }}\right)$. Based on the observed download 
rate, the client can adapt the quality of the next chunk such that it will be downloaded before its playback deadline. Whenever the observed download rate suggests that downloading chunks at the current encoding rate would lead to playback deadline violations, chunk requests can be made at lower encoding rates to avoid playback stalls. Similarly the encoding rates can be appropriately increased in case if the observed download rate suggests the opposite. In fact, similar to non-adaptive HTTP streaming, an additional buffer $T_{\text {min }}$ is typically maintained to avoid stalls. This is also taken into account when selecting video qualities of future chunks.

The rate-estimation and quality adaptation procedure discussed above is very simplistic. It is well known that network throughputs can vary drastically due to several factors, such as competing flows, packet drops, switches or routers operating under heavy loads. Throughput variation is more pronounced in the case of wireless access techniques such as LTE and $\mathrm{WiFi}$, where presence and movement of objects and environmental factors can degrade a signal and negatively impact the throughput. Therefore, rateestimation based on the observed download rate of a single chunk does not necessarily provide sufficient information about the throughput that would be available when downloading the next chunk. In practice, the expected download rate is extrapolated either based on a rolling window or a weighted average of several previous chunk downloads. For example, an exponentially weighted moving average might be used to calculate the estimated available bandwidth as $B W_{i}=(1-\alpha) \cdot B W_{i-1}+\alpha \cdot\left(S /\left(T_{\text {response }}-T_{\text {request }}\right)\right)$, where $B W_{i-1}$ is the estimated bandwidth during the previous iteration of the weighted average calculations, and the weight $\alpha$ determines the significance applied to the newly observed download rate.

The method and weights used to calculate the estimated bandwidth can be fine-tuned during run-time, to be more aggressive when a large buffer has been built up or to accommodate for a history of playback stalls by conservatively factoring down the estimated bandwidth, for example. In addition to the measured and estimated download rates, a HAS player can use other metrics to adapt the playback quality. For example, the number of dropped frames when playing a video can indicate that the CPU or GPU is unable to cope with decoding video frames in time. Other factors such as the screen size, state of the player (full screen vs minimized), platform (App vs browser) could also be taken into consideration.

As it is popularly referred to, HTTP-based Adaptive Streaming (HAS) is specified as a standard in the Motion Picture Experts Group- Dynamic Adaptive Streaming over HTTP (MPEG-DASH) [5] specifications and in the 3rd Generation Partnership Project (3GPP) [17]. The standards are loosely based on several commercial implementations of adaptive streaming solutions over HTTP. The most popular among these are Adobe's HTTP Dynamic Streaming (HDS) ${ }^{2}$, Apple's HTTP Live Streaming (HLS) ${ }^{3}$ and

\footnotetext{
${ }^{2} \mathrm{http}: / /$ www.adobe.com/products/hds-dynamic-streaming.html

${ }^{3}$ https://en.wikipedia.org/wiki/QuickTime
} 
Microsoft's Smooth Streaming (MSS) ${ }^{4}$. Among the three commercial implementations, Adobe HDS is open source while the other two are proprietary.

\subsection{Web caching and caching of HTTP-based streams}

Web proxies were initially designed as means to provide access to the Internet for a sub-network (subnet) consisting of several end-points. The web proxy acted as an aggregation point for all traffic from the network to the Internet. This facilitated the use of centralized firewalls and NATs at the proxies, while at the same time providing transparent Internet access to the end hosts. Web caching through the use of proxy caches was envisioned in the early 1990s [18]. These caches operate by storing a local copy of the content that goes through them. When a locally cached content is requested again, the cache serves the request from the local cache rather from the origin server. Web caching, as discussed before, reduces bandwidth consumption, transmission delays and the server workload. Inadvertently, web proxies also allow access to content even when the origin server is offline, allows for greater network utilization, decreased network traffic, and also provides central monitoring and management of the data flowing in and out of a network.

Caches are generally organized in a hierarchical manner at each level of the network. The most natural example for such an architecture would be to consider caches at client devices followed by caches at subnets followed by caches at the operator level organized at regional and national levels [19]. While distributed architectures for caching [20] have also been explored, hierarchical organization is most commonly used in practice. The predominant goal of proxy caches is to maximize the cache hit-rate. Hit-rate is defined as the the ratio of the number of requests served from the local cache to the total number of requests. The proxy cache has a finite data store or a disk on which it must organize and store content such that its expected hit-rate is maximized. Typically, content objects are added to the cache whenever they are requested and the cache does not contain a copy, and one of several cache replacement strategies is used by the proxy cache to chose whether to write over a piece of data or not. More intelligent cache insertion policies have recently been proposed to take into account the long tail of one-time requests [21], [22].

Some of the most popular cache replacement strategies are based on the Least Recently Used (LRU) policy or the Least Frequently Used (LFU) policy or hybrid policies called Adaptive Replacement Caches (ARC) which behave as a hybrid between LRU and LFU strategies. The LRU policy evicts an object that has been requested the least recently while the LFU scheme evicts the object that has the least access frequency. Several policies perform

\footnotetext{
${ }^{4}$ http://www.iis.net/downloads/microsoft/smooth-streaming
} 
trade-offs between cache hit-rate and byte hit-rate. Some policies might optimize cache hit-rate by keeping smaller objects for longer in the cache (Greedy-Dual Size Frequency) while others such as the Least Frequently Used with Dynamic Aging (LFUDA) store objects irrespective of their sizes to optimize the byte hit-rate. There are several trade-offs in this area and the choice of replacement strategy has to be fine-tuned based on the use case and the factor be optimized.

Web caching has played a significant role in the wide spread adoption of streaming over HTTP as the de-facto standard for video streaming. Web caches were initially developed to store and replicate web pages. Caching has been proven to be very effective for regular web traffic. Typical web requests go through multiple level of caches before actually reaching an origin server. For example, all modern browsers are equipped with a browser cache, commercial Internet Service Providers (ISPs) typically deploy several caches along their networks to reduce retrieval times, and even the actual web server might be present behind levels of caches in the content provider's network or a CDN.

When compared to receiving the response from the server, a response from the cache has a smaller RTT. This is mainly due to the caches being physically closer to the client and the round-trip distance that a packet has to travel from the client to the proxy is smaller. Proxies also often help avoid that the packet needs to go through potential transit bottlenecks. In general, these reduced RTTs will also result in faster downloads and streaming speeds. Asides from improving throughputs, presence of caches and also improves the server-side scalability. The cache effectively acts as a server if the content is found locally, hence offloading the server whenever cache hits occur.

ISPs and content providers' monetary policies depend heavily on the number of bytes sent and received to one another. For example, a larger provider ISP might charge its customer ISP based on the number of bytes it forwards to/from external domains, on behalf of the customer ISP. In this case, the customer ISP can reduce its operational expenses by installing several caches within its network, therefore avoid having to repetitively download the same content through its provider.

Since streaming traffic over HTTP is also web traffic, caches can also store and replicate chunks of HTTP streams. Whereas progressive streaming over HTTP would require the cache to store the entire video file in the local memory, this is not necessary with chunked video. This is important since video occupies large volumes when compared to regular web traffic, and users who watch a cached video often might not watch the entire content. If a cache choses to store entire videos, a significant portion of the cached data might therefore never be used at all. Likewise, for the cache to store a video, it requires that at least one client streaned the video completely, from the beginning to the end. With chunked or range-based HTTP streams, the cache can initially store any chunk that goes across the proxy and gradually 
discard the less popular chunks and replace them with more popular chunks or the chunks that are most likely to be requested in the future. By doing so, the cache can store other content that are most likely to be requested, and lets the cache to contribute effectively with a much smaller footprint.

Although proxy caches are highly beneficial for the clients, the network provider, and the service provider, the research presented in this thesis [6], [8], [10] and others [23], [24], [25], [26] reveal some drawbacks and challenges that must be addressed when maximizing the benefits and eliminating the drawbacks. Here, we broadly highlight one such challenge. First note that the encoding rate and the time at which requests are made are determined based on the buffer occupancy and the inferred network throughput during previous chunk downloads. As discussed earlier in this section, chunks allow proxy caches to contribute positively to a HAS session with only a few cached chunks. For example, when the client's request encounters a cache-hit, the response reaches the client much faster than it regularly would if the chunk were sent by the server. While this is encouraging, this faster chunk downloads lead to the client over estimating the available throughput. This can also potentially cause client to request a chunk at a higher encoding rate. As long as the client experiences cache hits, the client will likely continue to successfully download chunks in time for playback, but when the client encounters a cache miss, the skewed estimated bandwidth might not accurately reflect the available throughput to the server. Under such circumstances, the client might experience playback stalls if the buffer size or the buffer occupancy is too small.

Playback stalls and fluctuations in the video quality have been found to be important QoE metrics from the user's perspective [15]. It is therefore important to avoid playback stalls and perform quality adaptation in a gradual manner. It is also important to understand how HAS clients interact with the network, and the effect that HAS access patterns have on the network. In this regard, this thesis presents the design and evaluation of several proxy-assisted solutions, that are designed to aid in improving the viewers QoE. In addition to the proxy-assisted solutions, this thesis also discusses cooperative client-proxy solutions where the client and the proxy share information between one another in order to ensure that the decisions made by the client and the proxy are consistent and result in the best possible playback experience at the client.

\subsection{Other streaming techniques}

Several techniques have been explored previously to efficiently distribute multimedia content. The following paragraphs attempt to cover some of the most relevant techniques to the context of this thesis. To accommodate more users, provide a service with better audio-visual quality or with lower delays, scalable and efficient protocols are required. As video delivery over the Internet became a reality, several such techniques have been proposed 
and experimented with. Perhaps the first among these were the works based on multicast or broadcast domains [27], [28], [29]. Intuitively, multicast and broadcast based methods provide much better server-side scalability, as the server does not have to handle $n$ unicast streams but 1 multicast stream. IP-based multicast trees to distribute IPTV [30] content were developed to build an IP distribution tree from a source to several receivers. Here, designated nodes, called Rendevous Points (RPs), are used to effectively manage and maintain the distribution tree. The RP can connect to multiple sources and obtains content to disseminate via unicast from the sources. The distribution tree is dynamically built using the Internet Group Management Protocol (IGMP), where a set-top-box wishing to connect to a certain broadcast connects to a router and subscribes to a distribution tree. This architecture requires the network core to maintain state, where nodes wishing to participate in the distribution tree subscribe to the RP or to nodes above it in the distribution tree.

Scalable Video Coding (SVC), an extension to the H.264 Advanced Video Codec was envisioned as an adaptive scalable video delivery codec [31]. SVC supports adaptive screen sizes (spatial resolution), adaptive frame rates (temporal resolution), and bit rates (quality resolution). This makes it possible to adapt content based on different modalities where the spacial, temporal or quality resolution might be adapted on the fly in real-time applications [32]. When compared to HAS, SVC requires only one video encoding for several adaptation profiles while the number of video encodings required in HAS scales linearly with the number of adaptation steps in one direction. Video streams in SVC can be split into a base layer and several adaptation layers. The client can receive these layers in a best-effort manner and reconstruct content on the fly to adaptively present the video. However, the process of generating several adaptation layers involves a significant coding penalty [33]. Furthermore, a client-driven SVC over HAS would involve multiple requests for different layers for the same chunk. Each initial request would have to endure a connection setup, TCP slow-start and in cases where the connection idles for durations greater than the TCP Round-Trip timeOut (RTO) value, a idle-timeout overhead as well.

Availability and costs play significant roles in adoption and evolution of services over the Internet. Video streaming requires dedicated and globally distributed resources to store data and network bandwidth to disseminate content on-demand. CDNs play central roles in on-demand and live streaming services by storing and distributing vast amounts of data globally [34]. However, CDNs require large investments in order to install, manage and run the required infrastructure. On the other hand, Peer-to-Peer (P2P) based systems [35], [36], [37] rely on a group of clients sharing content with others. This system, as exemplified by BitTorrent, eliminates the need for servers. However, availability and download speed heavily rely on the number of clients which posses the requested content and their upload bandwidths. Owing to these limitations, in an on-demand or live VoD scenario, it is hard 
to provide service level guarantees using a pure P2P-based system. Hybrid approaches which use peer-assisted servers can be used to get the best of both worlds, where the availability and service level guarantees can be provided by designating a server which is aided by several peers to stream content to a client [38], [39].

The enormous data volumes involved in large-scale on-demand and live streaming require scalable, stable, cost-efficient and flexible solutions that can handle changes in demand and access patterns over time. Improvements in bandwidths available to end-users and developments in virtualization techniques have lead to commercial deployment of cloud-based services. These systems allow to remotely store, process and distribute large amounts of data. In addition to these, cloud-based systems also offer quick reconfiguration and resource allocation on demand. Cloud and peer-assisted streaming services however require careful consideration of different expenses and effectiveness of different techniques. For example, peer-to-peer techniques are very effective in distributing vastly popular content, while a purely server-based approach might struggle when it comes to a large number of clients. A service provider might chose to carefully distribute the process of content delivery over different techniques to optimally guarantee good user experience with lower running costs [39], [40].

\subsection{Interactive branched video streaming, a primer}

Browsing the Web has always been an interactive and personalizable experience. User interaction with links on the web pages, to a large extent determines the content that is shown to the viewer. Contrary to browsing over the Web, video streaming over the web has only been personalizable through video recommendations (as part of browsing) and actions such as fast forward and rewind. In most cases, the viewer is expected to watch a video from the beginning to the end. We believe this will change with interactive branched video streaming. In the following, we outline some of the key concepts and ideas behind our HAS-based branched video streaming system. While this design idea is a part of our contributions, we introduce some of these ideas here, so as to set the context for the remainder of the thesis.

Interactive story telling has been used in several novels, perhaps most notably in the popular children's novel series called "Goosebumps". The reader would simply turn to a different page based on the different options given by the author. Along similar lines, interactive video streaming is a concept where the viewer can interact with a video and influence the content that is about to be presented. Earliest digital applications of interactive videos appeared in DVDs where the viewer, by pushing a button could navigate to a different chapter in the DVD, thereby altering the story line 


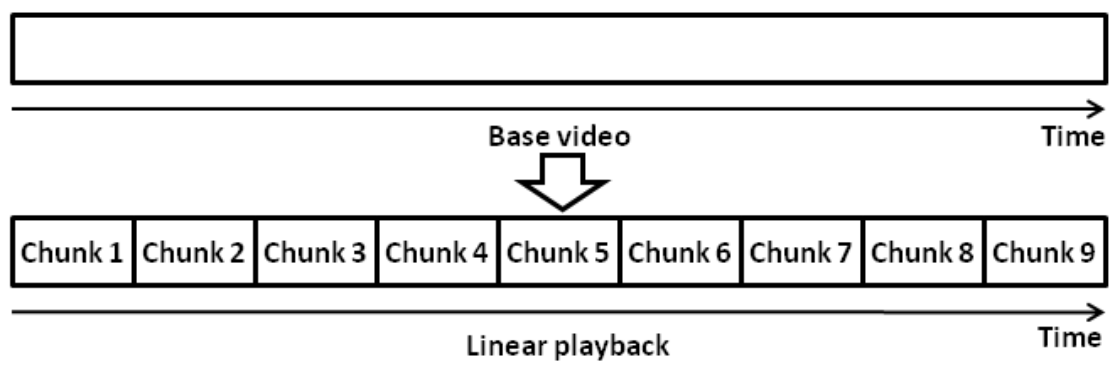

(a) Chunked video, linear playback

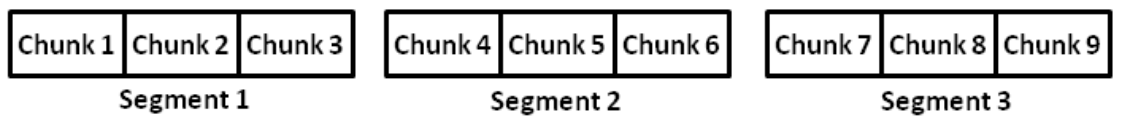

(b) Segments of contiguous chunks

Figure 2.4: Example linear video organized into segments for interactive branched playback

according to the choice made by the user. This class of interactive videos is referred to as interactive branched streaming or simply branched streaming from here onwards.

When compared to traditional progressive download over HTTP, chunk or range-based streaming over HTTP; in a way simplifies the process of branched streaming. Since the videos are split into addressable chunks or ranges with known playback durations, potential playback paths along a video can be designated which resemble graph or tree structure. User interaction, by means of clicking a button or key strokes can be used to determine branches to traverse with defaults assigned in case the user does not interact. Figures 2.4 and 2.5 illustrate the key differences between traditional linear HAS streaming and interactive branched video streaming over HAS, as proposed in our research [6], [8].

Figure 2.4a shows a chunk-based HTTP video generated from a base video. When this video is played back as a regular video, also known as a linear video; the chunks are played back linearly. Although the time at which chunks are requested can vary based on buffer occupancy and network conditions, the chunks will be downloaded in the same order as shown in the figure, unless the user initiates a VoD functionality, such as, fast forward or rewind for example. In contrast to linear streaming, nonlinear streaming involves multiple plot sequences within a video and places different requirements on the download and playback ordering of chunks. For example, consider an example where the the first three chunks of the video make up an introductory scene which is followed up by two alternative scenes, each defined by a sequence of three chunks $(4,5,6$ and $7,8,9)$. The 

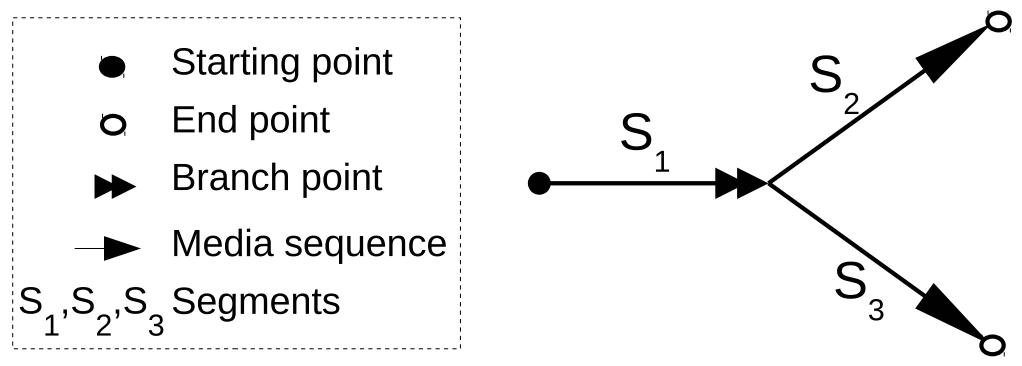

Figure 2.5: Interactive branched playback

user may chose to interact with the video anytime before reaching the end of chunk 3 (branch point), thereby choosing to play either the first set of chunks or the second set of chunks. From here onwards, these sets of chunks which form a part of non-interactive playback between two branch points are called as segments. Such an example scenario is illustrated in Figure 2.5. Here, segment S1 corresponds to segment 1 in Figure 2.4 and segment S2 corresponds to segment 2 in Figure 2.4 and so forth. Again, segment 1 is the initial segment to be played back, while segments 2 and 3 are branch options, whose playback and download ordering are uncertain. In order to facilitate stall-free playback after the branch point, at least the first chunks of both segment 2 and 3 must be available to the player to perform seamless interactive playback.

Avoiding stalls during playback has found to be one of the most important factors influencing viewer's perceived QoE [15]. Both the number and duration of stalls have been found to have a negative impact on user experience for linear videos. With regard to interactive branched video, avoiding stalls, especially during branch points becomes all the more paramount as the user look forward to a seamless playback experience.

In addition to regulating the buffer occupancy based on the currently played segment, as done by the regular HAS players (discussed in Section 2.3), an interactive nonlinear player will also need to account for the path structure and the potential segments that lie after the upcoming branch point. In addition to changes in the buffer management algorithms seen in classical HTTP-based streaming clients, an interactive branched client also requires special prefetching algorithms which determine the best time instances to prefetch chunks which are present after a branch point. These algorithms must download these chunks such that they are downloaded before the client reaches the branch point, while at same time ensuring that the playback buffer of the current segment does not drop too low, resulting in playback stalls. One of the major contributions of this thesis is to identify this problem and cast it into an optimization formulation. The formulation can be used to optimize the viewing experience.

The aforementioned example in Figure 2.5 is with a video that is available 
at a single encoding level. In reality, such a system must be implemented over HTTP-based adaptive streaming, where the chunks are available in multiple encodings as described in Section 2.3. To playback seamlessly and at the best possible quality as allowed by the network, the client must determine the best quality at which chunks of the current segments must be downloaded, and the best time instances and qualities at which chunks of branch options must be downloaded. A part of this thesis addresses these challenges encountered in implementing an interactive branched player over HAS.

Websites such as YouTube, which offers User Generated Content (UGC) have become very successful in the recent past. The success of UGC-based sites can be attributed to them aggregating the endless resources and creativity of regular home users. Owing to the prevalent acceptance and growth of Internet-based services in our households, several creators are interested to share their content with users all around the globe for a small incentive. These incentives are typically advertisement-driven revenue. Thereby, the success of a content creator depends on the number of views or clicks obtained by the content. UGC-based sites have now branched off into several domains ranging from professional and amateur multimedia sharing, question and answer websites, forums, wikis, podcasts and other forms of media. The growth and popularity of video streaming can also be partly attributed to UGC. Websites such as YouTube have over a billion users [41] and several professional creators and organizations publish content on such websites owing to their popularity and mass-media status.

The viewing public have vastly different tastes and preferences. Today's streaming websites rely on the viewer using $\mathrm{VoD}$ functionalities such as fastforward and rewind to navigate place themselves in the video timeline in accordance to their tastes. However as users, we know that such actions are often hit-and-miss and often lead to lost interest in watching a video. In fact, a vast proportion of all videos are never watched beyond a few seconds as users loose interest owing to a variety of circumstances [15]. However, a video can also be viewed in several ways, with slightly different story-lines and screenplays that might suit our tastes better. In order to facilitate a content creator (both professional and UGC content creators) to accommodate for the vast differences that one might see in the public, we envision a system where the creator distributes a base-video over professional or UGC-based sites. Viewers and secondary creators can make use of this base-video to create several interactive versions of this video that might better suit the general public's tastes.

However, such ideas might not be very scalable if they required every interactive version of the base-video to be stored on a server. In this thesis, we argue and see how interactive UGC-based videos can be made more scalable by the use of HTTP-based streaming, where the video is divided into chunks with unique URLs. An interested party can now generate an interactive version of this video by referencing different chunks of the video 
to different branches. The client, when playing back this video will download chunks in an order that is specified by the user interacting with the video or according to default path defined by the creator. The benefit of HTTP-based interactive branched video is that with a single base-video, several interactive versions of this video can be generated with only changes to the client-side playback algorithm. This thesis describes the design, implementation and evaluation of strategies required for interactive branched video playback, as well as provides a fully client-driven solution that allows for infinite customizability with no changes to the server-side architecture, content generation or content distribution techniques. 


\section{Chapter 3}

\section{Related work}

The recent and widespread adoption of HAS-based services has lead to a wide range of research related to the topic. This chapter discusses the most relevant research pertaining to the content in this thesis.

\subsection{Content delivery}

Video streaming over the Internet has long been awaited as the next big thing to emerge from the dot-com boom. Today, as a result of continuous improvements, network architecture and techniques used for video streaming are capable of achieving high-quality playback. This coupled with large content catalogs have resulted in very high usage of video streaming services. In fact, today, video streaming traffic dominates the application mix on today's Internet backbone, accounting for more than $60 \%$ of the overall traffic volume [1]. This share is expected to grow as streaming services penetrates into many more households. Currently most large-scale streaming services of high-quality content is done through HTTP-based Adaptive Streaming (HAS).

When designing systems for large user bases, it is important to make sure that large-scale content distribution is achievable with the least overhead. The system and protocols used must at the same time ensure that the protocol stack can inter-operate with other traffic, and allow the clients to consistently claim their fair shares. Although the goals of most content delivery systems remain the same, different architectures have varying characteristics, performance and workloads that they can handle [42].

Peer-to-peer networks, as exemplified by the now defunct systems Napster and Gnutella, or perhaps the more popular BitTorrent-like [35] systems of today, have relied on utilizing the distributed power available at end hosts to disseminate content. Such systems outweigh the traditional centralized 
client-server based systems in areas such as scalability, cost of establishing and distributing content, and redundancy. However, compared to the classical client-server paradigm, it is difficult to provide guarantees when delivering content over regular peer-to-peer networks. As means to address this, approaches such as cloud-assisted and peer-assisted content delivery techniques have also been proposed [38], [39], [40]. Such systems, in addition a centralized server or a CDN together with the power of end-hosts aiding the content delivery. These approaches makes sure that there is at least one copy of the content available at the server (good for availability), while at the same time, taking advantage of clients' upload bandwidth to improve scalability.

Yet another alternative to scale content delivery to large user-bases is by making use of Content Delivery Networks (CDNs). CDNs [43] use a network of interconnected servers, which are distributed strategically and geographically to ensure quick access to data stores from several parts of the world. Several large CDNs have a global presence, and are capable of quickly moving content form origin servers to replicas, which are located closer to user-bases. The cost of delivering content using CDNs has dramatically reduced in the recent years (by a factor of 10 between 2006 and 2010 [44]) and has thereby made CDN-based content delivery very attractive. In addition to decreasing operating costs, CDN-based content delivery offers reconfigurability, centralized management, admission control, utilization of web-caches and other advantages, thereby proving more attractive than traditional peer-to-peer networks.

Perhaps, the key benefit of CDNs, as opposed to other distribution techniques, in general, is that the $\mathrm{CDN}$ provider and the content provider are different entities. This allows both the providers to focus on their core business. A content provider can also purchase resources from a CDN and relinquish control of handling and moving replicas to the CDN. Such agreements are analogous to the Service Level Agreements (SLA) between autonomous systems to ensure reachability. Whenever a client from a certain part of the globe attempts to access a video from the content providers' website, it is redirected to an nearby CDN server that would provide good QoS, based on the current network load, client location and other parameters. This process is most commonly done through DNS resolution, where the client resolves a service or content to different CDN-edges based on the entries in the DNS and other DNS-based performance metrics [45]. CDNs also perform load balancing in accordance to the demand, user location, network characteristics and QoS, and is generally performed at the front-end or edge-server, which transparently fetches data through multiple back-end CDN servers [46]. Load balancing based on Software Defined Networking (SDN) [47] based approached have also been proposed. In addition to these, there are several components within the context of CDNs that are research fields in their own right, including server placement, request routing [48], replica selection [49], pricing, caching within CDNs, and many more [50]. 
With regard to video streaming, using CDNs to deliver content has now become the norm, especially for popular services such as YouTube, Netflix, Hulu, Amazon Prime and others. Given the global user-base and diverse access patterns, CDNs have been pivotal in on-demand streaming over the Internet gaining wide-spread acceptance. Some of the most popular CDNs include Akamai ${ }^{1}$, Limelight ${ }^{2}$, Level ${ }^{3}$, Windows Azure ${ }^{4}$ and CloudFlare ${ }^{5}$ to mention a few. However, it has also become common for Internet Service Providers (ISP), such as AT\&T and Verizon to deploy their own streaming services over their CDNs. The goal of such services is to attract subscribers towards their providers' streaming services, and thereby cutting down the need to obtain data from external CDNs, which might impact QoS and increase the monetary costs of the ISPs. Although these developments have blurred the lines between content providers, service providers, and CDNs, the dynamics between the different parties is one of the major forces that shapes the future of large-scale content delivery [51].

\subsection{Client-driven approaches}

The process of streaming a video over HAS is completely client-driven. This places a large significance on the client-side algorithms used to perform quality adaptation in HAS. Although several network level aspects, such as competition, congestion, and packet losses can influence the playback quality, the client-side adaptation logic can take into account these factors and strive towards providing the best possible playback experience, given the current circumstances. Client-side algorithms must also be robust enough to be able to obtain their fair bandwidth share, while at the same time be flexible to accommodate for the factors discussed above.

\subsubsection{Rate-adaptive streaming solutions before HAS}

Before HAS, several rate adaptation mechanisms using different transport and application level protocols were proposed. Some of these were clientdriven and others were server-driven. The server-driven mechanisms typically relied on feedback messages from the clients which were used to convey information about bandwidth, buffer occupancy [52] and other parameters of interest. While some protocols relied on application-level metrics [53], metrics from the transport layer have also be used for such purposes [14,54]. In general, server-controlled rate adaptation mechanisms have the disadvantage that the control loop will always lag behind the network condition by a factor of at least one RTT between the client and server. The delay might

\footnotetext{
${ }^{1}$ https://www.akamai.com/

${ }^{2}$ https://www.limelight.com/

${ }^{3} \mathrm{http}: / /$ www.level3.com/en/products/content-delivery-network/

${ }^{4}$ https://azure.microsoft.com/en-us/services/cdn/

${ }^{5}$ https://www.cloudflare.com/
} 
increase exponentially in cases where there are severe network congestion or packet losses. In addition, these mechanisms also require the server to maintain state information for every active client, thereby occupying more server resources. Other client-controlled adaptive multicast protocols have also been explored, where clients subscribe to one or more multicast groups [55].

Client-driven quality adaptation mechanisms were primarily explored in the context of scalable video codecs. By adding and removing enhancement layers to a video based on the relation between the consumption rate at the client decoder and the available bandwidth [56]. Other approaches which address the issue of playback quality adapting to the available network throughput and short-term network congestion under the broadcast domain have been proposed through receiver-driven layered multicast [57].

Since the early works on video streaming, there has been a shift from using UDP to TCP. Much of this shift is motivated by improvements to access speeds, larger client memories and gradual improvements to the delivery infrastructure.

Although downloads over TCP result in a saw-toothed throughput pattern, the short-term fluctuation in throughput can be accommodated for by using a larger client-side buffer. Furthermore, since TCP provides vital guarantees that are required for the Internet's scalability and stability, building either an entirely new transport protocol for streaming applications or moving some of these aspects to higher layers while using UDP seemed unnecessary [58]. This led to the wide-spread acceptance of streaming over TCP and eventually to streaming over HTTP using HAS.

Client-based solutions are natural when operating over HTTP and it has been shown that throughput over HTTP-based request response streams can scale well in relation to the available bandwidth, for example, using larger chunks or multiple concurrent downloads during high bandwidth conditions, it is possible to achieve fair and high bandwidth utilization while allocating bandwidths to clients [59].

\subsubsection{Client-driven approaches using HAS}

With regard to quality adaptation in HAS, the technique used to measure available bandwidth must be able to reliably build a picture of the network throughput over time. Most HAS clients make use of the average throughput measured at the application layer to generate these estimates. Alternatively, measurements at the transport layer can also be used, but the perceived throughput at the transport layer with TCP can fluctuate to a large extent as the protocol attempts to perform congestion and flow control. The practice of passing information from the transport layer to the application is also not encouraged as it might cause unintended loops in the network stack.

Several works have looked at generating reliable throughput estimates of the available network bandwidth. Most approaches make use of segment or chunk fetch times [60] and calculate smoothed averages of chunk download 
times, which in-turn (by knowing the size of downloaded chunks) can easily be used to estimate the available bandwidth. As described previously, HAS videos are divided into smaller pieces, which are called chunks or segments.

Chunk fetch time corresponds to the time taken from issuing the GET request for a chunk, to the time at which the $200 \mathrm{OK}$ response for that chunk has been received. Then, it can be noted that for the video to be stall free, the average encoding rate of the video that is downloaded must be no greater than the available throughput. In general, with adaptive streaming, the encoding rate at which chunks are downloaded are therefore adjusted based on the average measured throughput over HTTP chunks. Whenever additional bandwidth is detected, the client may chose to opt for a higher encoding, while under changing bandwidth conditions, the client may chose to switch to a lower encoding to avoid experiencing stall times.

Although the technique to maximize playback quality and minimize stall times by carefully choosing encoding rates for chunks has been realized in several commercial applications, a few shortcomings also exist to this method. Apart from understanding these aspects, it is also important to understand how video quality and playback artifacts affects user engagement and satisfaction. For example, research has shown that the buffering ratio (fraction of session duration spent buffering), playback interruptions in the beginning of a video, and overall the playback quality $[15,61]$ have a significant impacts on user engagement; both within a single session, and in user retention across different sessions. In addition, it has also been established that quality adaptation must be graceful; i.e., the magnitude of increase or decrease in the video's encoding rate should be within acceptable limits, and the frequency of occurrence of such events also should be kept to a bare minimum. Furthermore, the startup delay, defined as the time duration between initiation of playback and the time at which the client actually starts playing back the video, also should be kept as low as possible.

When discussing startup delay, it should be noted that HAS players start playback of a video only after the buffer exceeds a minimum level. One might chose a very low value for this parameter. However, this increases the possibility that the client might encounter stalls immediately after playback has began. Given that stalls during the beginning of playback sessions are much more likely to lead to abandonment, the value has to be carefully chosen such that the startup delay is as small as possible, while at the same time ensuring stall free playback.

In addition to the aforementioned requirements, it has also been found that smoothed averages of application level chunk throughputs do not necessarily reflect the true network condition and might lead to unfair bandwidth sharing in the presence of competing clients or flows [23, 24,62]. Since the presence of competing traffic in reality is the norm, such shortcomings must be addressed as well. 


\subsubsection{Interaction with TCP}

One reason for inaccuracies in bandwidth estimates is due to the way in which the abstraction layers the Open Systems Interconnection (OSI) model are organized. Within OSI, the lower layers serve the layers above them, and in addition each layer provides certain functionality which is completely performed within that layer. The notion of layers being independent of one another and performing critical services needed by the higher layers allows for transparency and provides interfaces on top of which applications can be built. HAS-based services and HTTP in general, uses TCP as its transport protocol. TCP provides functionalities such as reliable, in-order delivery, connection oriented communication, flow control and congestion control. Congestion control in TCP aims to avoid congestion collapse, while trying to provide the client its fair-share of network throughput based on the data path and the associated state of several network equipment along the data path [63]. TCP does not instantaneously provide a fair-share, but provides this guarantee over a period of time. In-fact, TCP congestion control typically results in a well known saw-toothed pattern based on an internal variable called cwnd or congestion window, which is updated regularly at the sender-side. cwnd specifies the maximum allowed difference between the number of in-order bytes transmitted thus far and the number of in-order bytes acknowledged thus far.

Similar to the saw-toothed throughput curve of TCP, HAS clients also have a distinctive on-off download pattern, as shown in Figure 2.2. This pattern depends on the receiver buffer parameters, namely, $T_{\max }$ and $T_{\min }$ and the client's quality adaptation logic. Broadly speaking, three different download patterns might arise from HAS players [64]. The simplest among these is the case where there are no off periods. The client simply downloads chunks back-to-back until it has downloaded the entire video. The other two cases contain either short or long off periods.

Contrary to the download patterns of HAS players, regular file downloads over HTTP are modulated only by the senders TCP's congestion control and flow control mechanisms. The additional control loop at the receiver's application layer with HAS clients is totally independent of the senders TCP's congestion control mechanism. This additional control loop, together with the periodic download patterns, (as seen in Section 2.2) and the lack of interoperability information between the sender and the receiver has been found to sometimes result in oscillations and inaccurate estimates.

TCP has several phases of operation. Under conditions where the available information is not sufficient or representative of the current network state, TCP falls back to a state called slow-start, where the algorithm tries to probe the network, first with a very small number of TCP segments and then increases the number of segments exponentially until a certain threshold is reached. When a HAS client decides that the buffer is sufficiently full and switches to the off state, the client remains at this state at least for a few seconds, where there are no requests from the client. Although HTTP 
1.1 [65] uses connection: keep-alive as default, in order to avoid reestablishing connections with the same server several times, the sender's congestion window value cannot accurately track the network state if there is no data transfer between the sender and the receiver. Under such circumstances, the TCP sender's congestion window is reduced to a preset value and resumes subsequent transfers from the slow-start phase. The timeout value which determines when to reset the congestion window variable depends on the round-trip time from the client to the server, and generally this value is in the order of a few hundred milliseconds in the worst case. Given that the HAS client remains in the off state for several seconds, the client has a penalty of resuming the download from slow-start every time it encounters an off period. In addition to the reduced throughput during off periods, the client now has to compete with other clients that remained active during the period the client remained inactive. These events often require several RTTs before TCP can reestablish a fair equilibrium. However, during this entire period, TCP throughput is lower than the optimal value and leads to the client estimating a lower application level throughput. A estimated throughput at the HAS client could result in the client resorting to a lower encoding rate, thereby affecting the viewer satisfaction. This can further hurt the client's throughput in the long run. In fact, it has also been shown that unfair bandwidth allocation and suboptimal quality choices can result from such scenarios.

To overcome issues with competing clients, and to ensure that the algorithms account for bandwidth and bitrate variability across a playback session, and, in general, to ensure that estimates are reliable and robust, different means to estimate the available throughput and schedule chunk downloads from application level measurements have been suggested. This includes randomized chunk scheduling through varying target buffer sizes, better statistical methods to estimate available bandwidths [66], control theoretic approaches to chose chunk encodings [67-69], network probing at the application layer [62], and objective-based optimization to ensure consistent playback quality [70]. Other paradigms have also been explored in addition to client-driven solutions such as server-driven traffic shaping [71] that can be used to ensure that clients avoid unnecessary oscillations. Another interesting approach to tackle the problem described above is to utilize the buffer occupancy as the primary indicator of the available throughput, rather than the measured download rate [72]. This approach, called the buffer-based approach, determines chunk quality levels as a function of the buffer occupancy, which in turn depends on the rate at which chunks arrive into the buffer and the rate at which chunks are drained from the buffer of the player. Evaluations have shown that the buffer-based approach can lead to robust quality adaptation mechanisms. However, during the start up phase the buffer occupancy does not accurately reflect the network conditions, and during these periods the clients can therefore fall back to a throughput estimate based scheme. 


\subsubsection{Standardization efforts}

In addition to client-side and server-side improvements, recent strides have also been made towards protocol standardization. The IETF has recently released the HTTP 2.0 specifications [73], largely based on SPDY [74], which is intended to provide improvements to the WWW in general. However, HTTP 2.0 has several additional features over HTTP 1.1 including, stream termination, server push, response multiplexing, and other features that can be beneficial in HAS scenarios as well. For example, the server push feature can be used to push chunks to the client that are most likely to be requested next, without having to wait for a request from the client, while stream termination can be used to close or terminate a request at a high encoding rate when the client determines that encoding rate to be unsustainable [75]. In addition to new application layer protocols, improvements to the transport layer have also been sought. For example, QUIC [76], is a UDP-based protocol that has been designed to provide features equivalent to TCP, including congestion control, connection oriented semantics, multiplexing with reduced latency, and include integrated security features and pluggable congestion control algorithms. Furthermore, both QUIC and HTTP 2.0 are designed for interoperability between one another. Finally, we note that TCP friendliness and improved loading times of QUIC have also been documented [77].

The growing trend of streaming video replacing broadcast television at households and increased mobile adoption is continually placing greater demand on the content delivery infrastructures. Although several aspects of delivery networks are over provisioned, the sheer scale and volume of traffic makes it increasingly difficult to design and improve the current state-ofthe-art. Large-scale measurements have shown that more than $20 \%$ of all streaming sessions have re-buffering ratios greater than $10 \%$, and that over $14 \%$ of the sessions have a startup delay of more than 10 seconds [78].

The content delivery infrastructures have time varying performance, depending on load, congestion in the network, outages, and other aspects. These variations contribute significantly to client-side artifacts. At the scale of the Internet, it would be challenging for a client to obtain fine-grained information about operating conditions of several CDN replicas from which it could obtain content to chose a replica based on the information, and continue following the state of the chosen replica over a playback session and make changes, if necessary. It would therefore be much simpler if there was a central controller, that would direct the client to the replica that provides the best performance, performs resource reservations, provides access control, and manages global resources under flash crowd events or network outages. Proof-of-concept and feasibility of such a centralized controller has been demonstrated through previous works $[79,80]$. In fact, the increased adoption of Software Defined Networks (SDN), suggest that future largescale control planes might run SDN-based controllers to perform resource and user QoE oriented optimizations. 
Several websites that perform video streaming over HTTP have emerged lately. This include both commercial and open-source implementations, many of which have been studied under both fixed and mobile networks [25, 81]. Among the popular HAS clients today, Microsoft Smooth Streaming (MSS), Netflix, and YouTube's source codes are not public. In contrast, Adobe's OpenSource Media Framework (OSMF), GPAC Project on Advanced Content, and DASH.js are some popular open-source implementations of HAS players. Several previous works that have studied commercial HAS clients have relied on packet-level traces to decipher the quality adaptation algorithms of commercial players.

HAS can also be used to perform live streaming. When comparing traditional live or broadcast television, where content (or video chunks) is pushed to the client, live streaming over HAS relies on the client pulling content from the server. HTTP is a pull-based protocol, and although HTTP 2.0 supports features such as server push, we detail the current practice of live streaming over HTTP.

At a high level, live streaming over HAS is very similar to on-demand streaming [82], where the client initially downloads a MPD file, parses it, and initiates playback by requesting chunks one after another. Under a live scenario, any new client must be able to decipher the 'live' chunk, i.e, the chunk which must be downloaded to start playing the live stream from an MPD file. This is achieved by continually updating the MPD file. Clients might also have to refresh their MPD files at times when some parameters of the stream might change. Chunks are generally numbered incrementally; thus, the client can now also rewind to an earlier point in time. A client side buffer is also used to guard against download jitter and throughput variations. Clients can also be staggered with relation to the actual live stream based on their buffer sizes. Live streaming over HAS also follows an on-off download pattern. In addition to the fairness and stability issues faced by on-demand clients, it has been shown that under live streaming over HAS, multiple client can easily behave in a synchronized manner, thereby leading to resource utilization issues [83]. Solutions to tackle such problems include distributing requests over time.

\subsection{Proxy-driven approaches}

HAS has been able to achieve wide-spread acceptance and deployment in the real-world due to its compatibility with the existing infrastructure, including the ability to distribute HAS content through CDNs and offloading servers through hierarchical cache deployments. However, HAS differs from traditional web-based services in several aspects. Perhaps the biggest differences are the sheer volume of video data when compared to other forms of web traffic (which typically are smaller in size) and the client-driven quality adaptation based on the perceived throughput conditions.

Although web-caches can offload servers and improve retrieval times, the 
client cannot differentiate if the chunk was delivered from the origin server or from an intermediate proxy cache. First, note that the client perceives differences in throughput rates and performs quality adaptation based on a long-term average throughput. The RTT to a web server is much larger than the RTT to a proxy cache, mainly due to shorter transmission delays. It is also well known that TCP's window-based congestion control mechanism allocates throughput to a connection proportional to the ratio of window size (W) and the RTT. Therefore, a chunk downloaded through a cache hit will be downloaded much faster when compared to a similar sized chunk that has been downloaded from the server. A series of cache hits might lead the client to estimate a larger available throughput. While the cache might contain future chunks at the current encoding rate, it might not have cached chunks of a higher encoding rate. This might expose the client to a much larger RTT (to the server), and might result in large retrieval times and even playback stalls. The interaction between HAS, existing infrastructure, and other factors such as cross-traffic [26] must therefore be understood to deliver high-quality content. In this thesis, we argue and show the value of passing additional information between the client and the cache.

The request patterns of HAS traffic can also be leveraged to improve the efficiency of caches and edge-servers. The process of prefetching, which involves predicting and downloading content before it is actually requested has been shown to be highly beneficial to traditional web-based services [84]. Along similar lines (as shown in Chapter 4), prefetching can be beneficial for HAS streams when RTTs are large and large volumes of data are downloaded. In addition to prefetching content to caches, cache replacement strategies also play a critical role in having chunks available for future requests. The quality adaptive nature of HAS clients require specialized cache replacement solutions as well. Previous works have looked into areas of cache replacement [85] and prefetching strategies for caches [86] in the case of quality adaptive videos, segment-based caching and prefetching [87] and, prefetching under the context of layered quality adaptation [88] as seen with SVC. This thesis proposes prefetching strategies for caches, which are designed with HAS download patterns in mind. These strategies are designed to improve the cache hit-rate, while also considering bandwidth constraints.

Over the past decade, the Internet has undergone tremendous changes. This includes significant changes in access patterns [89], user behavior, and the content that is distributed over the Internet. Any access over the Internet relies on host-to-host communication, in which content is retrieved from hosts associated with unique addresses. However, today's Internet is dominated by content and content-based architectures [90] have been proposed. Such paradigm shift from using host addresses to access content to content-based addressing has the potential to simplify several cache-related issues in today's networks as it decouples location and content. Although Content Centric Networks (CCN) have not yet gained popularity, it remains as one potential design path for a content-dominated Internet of the future. 


\subsection{Content personalization and interactive branched video}

Apart from a massive increase in the number of users and increased capability of networks to support high speed access to data, the Web has also undergone significant changes with the way in which content is generated and presented to the user. Until a decade ago, content that was available on websites were primarily generated either by the owner of the website, their employees or by professional content creators who agree to release their content through a certain website. This scheme followed the traditional content generation and distribution schemes that were being used by the printed media and by broadcast television. Although this ensured that the content is of high quality and facilitated users to transparently switch a different distribution media and yet get access to the same content, the volume or the sheer catalog of content that could be generated was sparse. However, this paradigm slowly shifted to give way to today's User Generated Content (UGC) based websites. UGC relies on the audience or users to create content that could be shared among the public. Any individual interested in creating and sharing any form of content could showcase their creations and even generate a fair bit of revenue based on the number of users they manage to attract.

The UGC-based distribution landscape consists of several stake holders. The content creator generates content which is placed on a website. The website, in addition to providing access to the content, also places several advertisements along with the content. These advertisements are paid for by companies interested in publicizing their product, based on an agreement between the company and the website. This content is presented to viewers, who asides from watching the content, might also explore products presented in the advertisement as well. The content creator is given a slice of the revenue received by the website based on the number of views, number of clicks on ads and other metrics which are agreed upon by the creator and the website. A part of the revenue is also used by the website to pay for operational costs. This model has now become one of the most common means of revenue generation on UGC sites on the Web.

Asides from leveraging the creativity of users from several households, UGC also partly ushered in personalization on the Web. It quickly became apparent that the advertisement based revenue model would be attractive to the viewers and companies only if the website could customize the content that is presented to the user, based on individual tastes and preferences. To personalize both the content presented and the advertisements, websites keep track of user's viewing history, location, search history, social networking and other information. This notion of personalization based on historical data, has now been incorporated into several professional websites, and now form a crucial part of the user experience on any website.

The overarching need to personalize and customize content and adver- 
tisements on a per user basis has led to the development of several recommender and personalization systems. Broadly speaking, recommender systems, which are predominantly used in web commerce applications can be categorized into three classes [91], namely manual decision rule systems, collaborative filtering systems, and content-based filtering agents. Manual decision rule systems allow administrators to specify rules manually based on the observed demographics, searches, or user profiles on the website, while collaborative filtering systems obtain explicit information from users on their preferences. This information is then correlated and filtered with the content presented to the user. Content-based filtering agents obtain personal profiles based on usage history and use this information to personalize content shown to the user. In general, today's system mine user data to customize and personalize web pages for users. Prior to personalized videos, content personalization and product recommendations based on user profiles were very common on web-commerce sites such as Amazon [92] and others. Such algorithms are designed mainly to retain users on a website and to promote relevant products as well.

Over the last few years, the mobile web has also taken off. Powered by significant improvements in mobile connectivity and capability of handheld devices, usage patterns have shifted from desktop-only to increasing use of mobile devices. This has led to further personalization opportunities. Hand-held devices typically are multi-functional and come equipped with several additional sensors that were not always present in a desktop machine. Most importantly, the location tracking capability and connectivity to the Web even during long stretches of mobility remains to be the major selling point of mobile devices. These developments have led to the development of Location Based Services (LBS) that provides high-quality, relevant, timely, and location-based services, features and information [93]. Services such as Foursquare ${ }^{6}$, Google Now $^{7}$, tripadvisor ${ }^{8}$ and many more have emerged lately which provide location, user interest and user historybased recommendations.

Similar trends based on personalized recommendation and customization to user's viewing preferences have been observed across most popular streaming websites. Video recommendations become all the more important in services with a large video catalog, as users might find the task of finding interesting videos to watch very daunting otherwise. The recommendation systems are also integrated together with the built-in social network functionalities. For example, using third-party authorization [94] through websites like Google or Facebook, a signed-in user can like, up-vote, comment or share videos to other friends. This information is used in addition to user search queries, watched videos, viewing time of videos to generate a list of recommended videos. YouTube's recommender system [95], makes use

\footnotetext{
${ }^{6}$ https://foursquare.com/

${ }^{7}$ https://www.google.com/landing/now/

${ }^{8}$ http://www.tripadvisor.com/
} 
of co-visitation counts of pairs of videos (i.e., videos that were co-watched withing a specific amount of time by other users), and generate a recommendation list as the most popular co-watched videos. This information can be combined with user profile information to generate a user specific recommendation, which can be ranked according to video quality, user specificity and diversification of viewer's taste.

Several approaches have been proposed for recommendation systems. At a high level, these systems obtain data which represents the behavior of a user or a user demographic under specific use cases. This data is then mined using machine learning techniques to generate a list of recommendations. The success of the generated recommendations are continually evaluated to allocate more weight to measures which resulted in the recommendation being used by the user. In addition to generating a list of recommendations, the system has to also rank the results to improve user retention. These requirements have lead to the development of content and context aware recommendation solutions [96,97]. Techniques for recommender systems, not only pertaining to matrix propagation [98], graph propagation [99], association rules [100] and machine learning based approaches such as SVM [101] have been proposed.

Several personalization techniques used today rely on collecting, storing and processing information gathered using the wide array of sensors available on modern smart phones. The wide spread acceptance of smartphones also makes it possible to use the inbuilt sensors on such devices to sense user's mental state and emotions and suggest actions or recommendations based on this information [102].

Personalization refers to customization of the content presented to the user, personalization of the content itself. For example, in the context of video streaming, personalization can be used to suggest the content that the user might be interested in and to allow the user to chose at fine granularity, the content and streaming paths that are actually being shown, as well as to provide customized path sequences or path-options for individual users. The most common means of video playback involves playing back the content of the video file linearly. While watching a linear video, the viewer can chose to introduce non-linearities in the video by either fast-forwarding or rewinding the play-point. In non-linear or interactive branched media playback, the video is still represented as a linear sequence of frames, but the client may interact with several objects in a video, thereby choosing to traverse to a different play-point. In order to achieve on-demand nonlinear playback, the user needs an interaction mechanism with the device playing back the content. In addition, the device must also be capable to parse the potential branch options that are available, and download content along all potential branches so as to facilitate stall-free interactive branched streaming, regardless of which path option the user selects.

In this thesis, we show how chunking and quality adaptation of HAS videos can be used to formulate effective personalization of such branched 
video. In particular, we leverage that in HAS, every chunk can be decoded independently of chunks before or after it. By adapting the quality of the chunks prefetched along different potential branches, given the current bandwidth budget, seamless playback can be achieved.

In the past, non-linear and interactive playback have been well investigated in the broadcast and multicast domains, where the client may subscribe to one or more channels and dynamically switch between channels [103105]. Under such scenarios, it is important to balance the server bandwidth and client path-diversity; i.e., the different paths that a viewer might chose while watching the same video. In contrast, with on-demand interactive branched streaming over HAS, the client downloads content from the server using a unicast connection, and the bandwidth usage is typically not negatively affected by high path diversity (except the additional prefetching that it may require). Similarly, streaming of non-linear datasets, such as, $3 \mathrm{D}$ graphics and visualizations have also been studied under the broadcast domain [106].

Branched video streaming has also been studied outside the contexts of HTTP-based adaptive streaming and broadcast or multicast-based streaming. Typically in these systems, the client uses progressive download, coupled with prefetching strategies and algorithms designed to parse user input and the video's structure [107]. The main drawbacks with such systems are the lack of quality adaptation, which might lead to large stall times due to bandwidth fluctuations and waiting times when performing interactive actions. Delays in executing interactive actions can affect the user satisfaction in drastic ways. As means to reduce such events, interactive-content-aware proxy caches (proxies that can parse the location of branch choices and attempt to cache the most popular branches always) and caching strategies specific to interactive branched streaming, similar to HAS-aware proxies that we propose in Chapter 4, have also been proposed [108], [109].

Prior works have also looked at developing formalisms required to generate and consume personalized videos [110], authoring tools for interactive branched videos [111], [112] and dynamic video composition systems [113]. These works look at generating and conveying information about personalized videos which can be created using GUI's or search queries.

Interactive branched streaming can also be defined in terms of multi-view streaming or 360-degree videos. Analogous to interactive branched streaming, the user interacts with the video to perform actions. Rather than telling a story through traversing different branches in a video, in multi-view and 360 -degree videos, the user navigates a scene through multiple cameras or viewpoints. Previous works have looked at tackling similar problems through coding [114], [115], making use of P2P networks for delivery [116] and distributed system design over IP networks [117] have also been proposed. Finally, interactive streaming for 3D videos, have also been studied. Such systems typically involve viewer head-tracking, prediction and prefetching alternative views to maintain smooth playback experience [118], [119]. 


\section{Chapter 4}

\section{Effects of proxy caches on HAS streams}

\subsection{Introduction}

For the last two decades video streaming has been expected to be the next killer application. Today, popular services such as YouTube and Netflix are offering large catalogs of user generated and professional videos, respectively. Video streaming has finally become mainstream, playing an increasingly important role in our everyday lives. It was recently reported that streaming media is responsible for close to $60 \%$ of the downstream traffic in production networks, and it is predicted that streaming media will account for twothirds of the traffic in the coming years [1].

While much of the early video streaming work focused on UDP-based solutions, today the majority of streaming traffic is delivered using HTTP over TCP. Use of HTTP enables both simple firewall traversal and effective content caching. These two factors provide immense benefits, as HTTP can reach almost any user, and content can easily be replicated at locations closer to the client.

With basic HTTP-based streaming, the video encoding that is delivered to a client is fixed at the time of the client request. To increase the quality of service and to better utilize the available network bandwidth, HTTPbased Adaptive Streaming (HAS) is being increasingly adopted by content providers [120]. With HAS, the video is encoded into different qualities and clients can at each point in time adaptively choose the most suitable encoding based on the current conditions.

While content caching plays an important role in the motivation for HTTP-based streaming, only very limited work considers the impact of using proxy assistance for HAS. 
In this chapter we present a performance evaluation of proxy-assisted HAS. Our experimental framework is designed using a popular open source proxy and a popular open source media player. Careful instrumentation allows us to measure the actual performance seen at the client, while controlling the network conditions and protocol parameters. We evaluate a range of different policy classes that may be employed in proxy-assisted systems and provide insights to the effectiveness of different policies and their performance tradeoffs.

While the general proxy policy classes considered in this chapter are applicable for any chunk-based HAS protocol, in our evaluation we use Adobe's Open Source Media Framework (OSMF), together with the accompanying (open source) Strobe Media Playback (SMP) media player. In addition to capturing the actual performance seen on the client, using an open source player also allows us to easily consider the impact buffer sizing at the client has on proxy solutions, and avoids other issues that may come with using proprietary players (e.g., having to decipher encrypted manifest files [25]). Our proxy-assisted policies are all implemented using open source Squid proxies.

Leveraging our experimental framework and instrumentation, the chapter makes three major contributions. First, we carry out an experimental evaluation of the latest version of SMP. Our results illuminate several important aspects of SMP performance, in particular relating to player buffer sizing.

Second, we analyze the player's performance in a proxy-assisted environment, considering a wide range of proxy policies including both basic baseline policies (such as a best-effort proxy that only caches chunks that it has served other clients in the past), and more advanced content-aware prefetching policies in which the proxy prefetches and caches chunks likely to be requested in the future (such as the chunk following that last requested by a client, at the same quality level).

Performance with the alternative proxy policies is found to be quite sensitive to the network conditions. If the bandwidth bottleneck is between the client and the proxy, we find that a relatively simple proxy solution can provide most of the potential benefits, but these benefits are modest. However, if the bottleneck is between the proxy and the server, caching at the proxy can yield a substantial benefit, but the limited bandwidth between the proxy and server must be more carefully utilized. There is traffic overhead associated with prefetching, and aggressive prefetching can therefore result in performance degradation. For example, a client that sees high hit rates at the proxy can be lured into selecting a higher quality than the proxy is able to serve if the future chunks requested by the client are not available at the proxy.

Third, we present a novel collaborative client-proxy framework in which clients and proxies share information with the goal of improving both the cache hit rate at the proxy, and the video quality at the client. Our per- 
formance evaluation results indicate that this goal can be achieved, yielding substantial benefits in some cases. Our buffer-size experiments also suggest that advanced policies are particularly attractive when assisting wireless clients with smaller buffers, for which unnecessary bandwidth usage may be more costly.

\subsection{Background}

Here we review the most relevant background for this chapter.

\subsubsection{HTTP-based adaptive streaming}

With HTTP-based streaming, either the quality level is statically chosen when the video is requested, or adaptively varied during playback. We refer to the second approach as HTTP-based Adaptive Streaming (HAS).

Typically HAS protocols use some variation of either chunk requests or range requests. With a purely chunk-based approach, used by OSMF and Apple's HTTP Live Streaming (HLS), for example, each encoding of every video is split into many smaller chunks. Each chunk is given a unique URL, which the clients can learn about from the video's manifest file. At the other end of the spectrum, e.g. used by Netflix, each encoding of a video is stored in a single file and clients use standard HTTP range requests to download ranges of bytes from these files. Hybrid approaches are also possible. For example, Microsoft Smooth Stream (MSS) uses a unique URL in the manifest file to specify each unique chunk, but then applies a serverside API to map chunk URLs into file ranges.

In this chapter we consider chunk-based adaptive streaming, as used by the Open Source Media Framework (OSMF). OSMF is open source and allows us to modify and instrument the player used in our experiments to directly measure the performance seen at the player. OSMF is a widely used design framework that includes a library of the necessary components to create an online media player. It also comes with the Strobe Media Playback (SMP), a fully pre-packaged media player for deploying OSMF. Other open source players include a VLC plugin [121] and GPAC [122]. For simplicity, here we use OSMF and SMP interchangeably.

\subsubsection{SMP overview}

\section{Buffer management}

Similar to other HAS protocols, including previous versions of OSMF, the most recent version of the SMP media player (version 2.0) controls content retrieval based on (i) its buffer occupancy, and (ii) the estimated available bandwidth. With OSMF's HTTP Dynamic Streaming (HDS) protocol, each 
encoding of a video is divided into smaller chunks that typically have a 25 seconds playback duration. The player issues requests for these chunks successively and the server delivers the chunks statelessly to the client. The protocol uses two buffer thresholds: the minimum $\left(T_{m i n}^{b u f}\right)$ and the maximum $\left(T_{\max }^{\text {buf }}\right)$ buffer time threshold.

During normal operation, the player tries to maintain at least $T_{\min }^{b u f}$ (in seconds) buffer occupancy at all times, and begins playback only when there is at least $T_{\min }^{b u f}$ seconds of data in the buffer. Generally, the player downloads until $T_{m a x}^{b u f}$ seconds of video is buffered, at which point it stops download until the buffer occupancy is less than the lower threshold $T_{m i n}^{b u f}$ again, at which point download is resumed. In this way, the player's buffer occupancy oscillates between these two threshold values.

\section{Rate adaptation}

Rate adaptation requires estimation of the available bandwidth (i.e., achievable download rate). This is accomplished using a weighted (rolling) average over the two most recently downloaded chunks. After download of a chunk, the player calculates the average download rate for that chunk and updates its estimated current download rate.

When downloading the next chunk, the most recent estimates of the available bandwidth are used to identify a list of candidate encoding rates that are sustainable for that bandwidth. For each candidate encoding, a reliability value is calculated based on the number of switching decisions of different types that have been made within a chunk-count-based window and the number of stalls encountered at these encodings. Among the encodings with a reliability value higher than a minimum threshold, the player picks the encoding with the highest reliability value. If there are no such encodings, then the lowest quality is chosen.

In addition to the above core mechanism, there are additional emergencybased or threshold-based rules that impact the player's decisions. These high-priority rules are designed to ensure that the player is not too aggressive/conservative, and include rules that prevent choosing a lower quality when the buffer is sufficiently full (threshold-based rule), and event-triggered (emergency) rules that keep track of empty buffer occurrences, dropped frames, and quality up-switches.

\subsection{Methodology}

This chapter evaluates HAS performance as seen at the client player, under different player and proxy policies, system configurations, and/or architectures. In contrast to prior works, which infer the player behavior based on network measurements $[25,26,81]$, we evaluate the actual performance seen at the player. By instrumenting the player we can avoid any potential mis- 
interpretations due to TCP buffer management and/or other hidden factors over which the player may have limited control.

\subsubsection{Experimental setup}

We implement and run a server, proxy, and client on three separate machines, all connected over the same $100 \mathrm{Mbit} / \mathrm{s}$ LAN. To emulate a wide range of network characteristics, including wide-area content retrieval, we use dummynet [123] to control the network conditions between the client and proxy, as well as between the proxy and server.

The server machine is running a Flash Media Server and is hosting a 10 minute video available at four encoding rates: $1300 \mathrm{Kbit} / \mathrm{s}, 850 \mathrm{Kbit} / \mathrm{s}$, $500 \mathrm{Kbit} / \mathrm{s}$ and $250 \mathrm{Kbit} / \mathrm{s}$, with key frames set four seconds apart. The key frame distance is vital in terms of the player's quality adaptation as rate switching can take place only at key frames. We have chosen encoding rates and key frame separation to keep the processing at the client and server modest. This allows us to focus on the network and proxy-related aspects. Levkov [124] provides a detailed discussion on the tradeoffs in picking a good key frame distance. Finally, we use open source Squid proxies to evaluate a range of different proxy implementations/policies.

\subsubsection{Player instrumentation and metrics}

For the purpose of player evaluation, we instrument the SMP media player to output important events and parameter information to log files for later analysis. This allowed us to capture the current state of the player at almost every player event, including when a new chunk is requested, when a decision is made to change the quality level, when observing server responses to rate-switch decisions, as well as at the times of other more subtle client-side events. For each such measurement, perhaps the most important information collected is the current buffer occupancy (defined as the amount of video content that is in the player buffer, measured in seconds) and video encoding rate.

In order to characterize the player performance we used the log files from our instrumented client player to calculate a set of metrics that capture both differences in performance and general adaptation characteristics.

- Quality level: The fraction of time the player spends at each quality level during playback.

- Quality switches: The number of quality level changes that the player makes per minute.

- Stall time: The total time the player is stalled.

- Buffer interruptions: The number of times per minute, regardless of how short, that the player is interrupted during playback, due to lack of data in its buffer. 
Unless stated otherwise, throughout this chapter we present average values (with $95 \%$ confidence intervals) calculated over at least 10 full experiments. We also consider proxy-specific metrics, such as the hit rate, defined as the fraction of the requested chunk that are found in the proxy cache, and the corresponding bandwidth savings due to these chunk not having to be obtained from the server. The caching decisions made when serving one client can impact subsequent clients, and for this reason, in some cases, we show performance conditioned on the number of previous clients that has viewed the same content as the current client. Finally, to provide additional insights, we occasionally refer to the raw event logs.

\subsubsection{Scenario-based evaluation}

In our experiments we use both synthetic bandwidth traces that capture artificial baseline bandwidth scenarios, as well as real-world bandwidth traces that capture the bandwidth dynamics in four example environments. In all scenarios, we use dummynet to control (i) available bandwidth, (ii) loss rate, and (iii) round-trip time (RTT). This control can be exercised so as to emulate the characteristics of a network bottleneck between the client and proxy, and between the proxy and server. While this chapter only shows a representative set of example results, in which we typically change one factor at a time, while controlling the others, we note that we typically used full factorial experiments.

\section{Synthetic traces}

To study the impact of the available bandwidth and how quickly it changes, we use artificial baseline scenarios. Figure 4.1 illustrates two example scenarios, with slowly and rapidly varying available bandwidth, respectively. We also use a third scenario with static available bandwidth. The average bandwidth $(725 \mathrm{Kbit} / \mathrm{s})$ is the same in all three scenarios, and the total time spent at each bandwidth availability level is the same in the scenarios with slow and fast bandwidth variations. In fact, for these scenarios we picked the average time spent at each level to be the same for all levels, such that in the ideal case (in which the player always picks exactly the quality level that is the highest possible based on the current available bandwidth) the player would spend exactly the same amount of time at each quality level. Of course, this is not expected to be achieved in practice.

\section{Real-world traces}

The real-world bandwidth traces were collected and shared by Riiser et al. [81]. The traces capture the available bandwidth seen in a 3G UMTS network in different parts of Oslo. Each trace represents a different mode of transport (bus, ferry, metro or tram) and allows us to provide insights into 


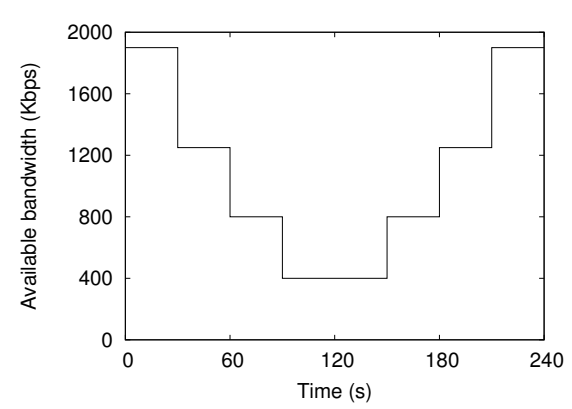

(a) Slow variations

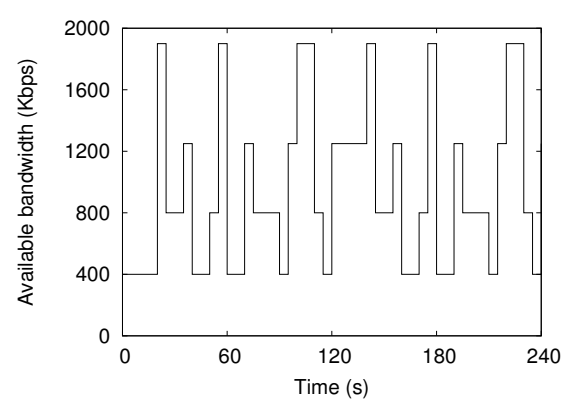

(b) Fast variations

Figure 4.1: Synthetic baseline traces for the available bandwidth

how the player performance is influenced by different geographical and physical factors. Figure 4.2 shows the available bandwidth under the different scenarios.

Each of the four traces exposes the player to distinctly different conditions and variations in the available bandwidth. The high available bandwidth in the bus scenario often is well above our highest encoding rate. The ferry route is interesting in that it illustrates a scenario in which there are highly different conditions, depending on the client location. When the client initially is on land the available bandwidth is high, the observed bandwidth is low while on the ferry, followed by high availability when back on land. These changes are clearly visible in the bandwidth plot. It is interesting to note here that the available bandwidth on the ferry is lower than our lowest available encoding rate while the bandwidth observed on land is very good. The metro trace captures the available bandwidth as the train initially is above ground and then enters a tunnel, as reflected by the abrupt change from an available bandwidth in the 500-1400 Kbit/s range, to a period with poor bandwidth availability. Finally, the tram trace shows relatively constantly time-varying bandwidth availability. It is important to note that the limited bandwidth availability in both the metro and tram traces may suggest that these clients should not be expected to view the video at our highest encoding rates.

\section{Loss rate and RTT}

To capture the impact of loss rates and round-trip times, for each of the above scenarios, we use dummynet to analyze five sub-cases of each of the above scenarios. In the basic case of a single client-server connection, these can be summarized as follows: (i) no additional delay or packet losses, (ii) no delay and $2.5 \%$ packet loss rate, (iii) no delay and $7.5 \%$ packet losses, (iv) 50ms RTT and no packet losses, and (v) 200ms RTT and no packet losses. Note that the first case corresponds to the ideal loss/delay condi- 


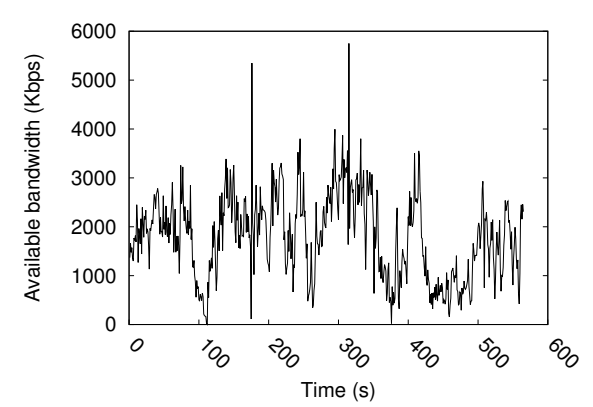

(a) Bus

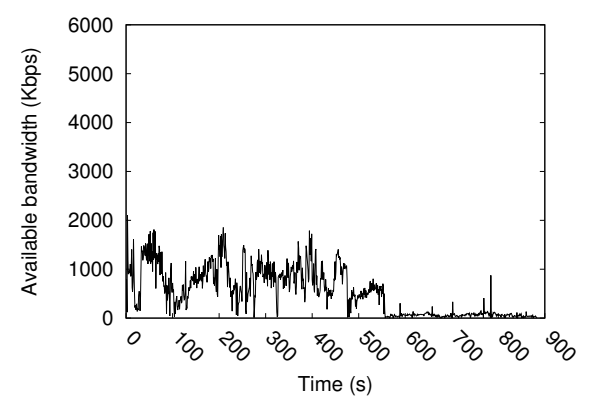

(c) Metro

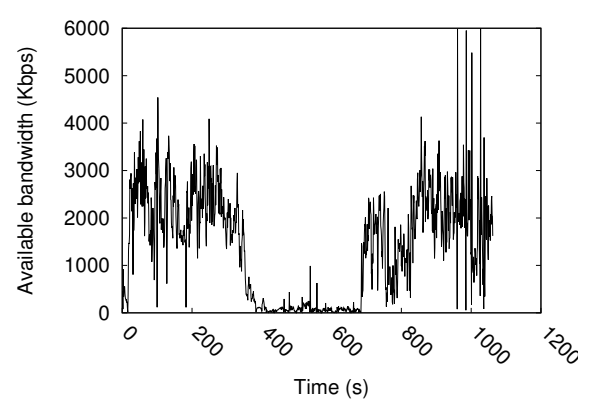

(b) Ferry

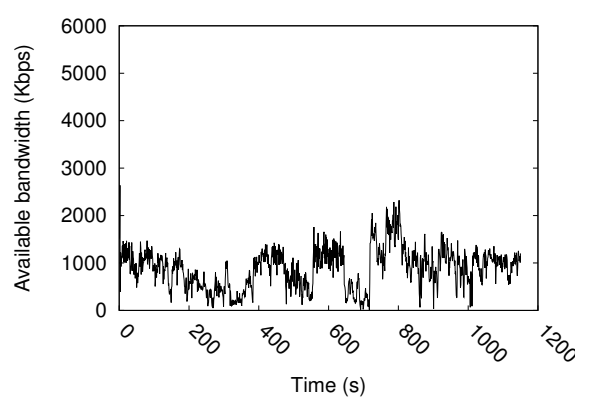

(d) Tram

Figure 4.2: Real-world traces of available bandwidth

tions, while other cases may be more realistic. The use of $2.5 \%$ and $7.5 \%$ packet loss rates are selected as they may be representative of acceptable packet loss rates in wireless environments. The RTT values are based on intra and cross-continental traffic. To put the RTT in perspective we performed traceroute measurements to the top million websites according to alexa.com on June 12, 2012. These measurements suggest that a client on the Linköping University campus would see an average RTT for these sites of $177.4 \mathrm{~ms}$ (and an average hop count of 17.74).

\subsection{Player characterization}

Before considering the impact proxies have on the player performance, it is important to first build an understanding of the player characteristics and baseline performance. As proxies potentially may cause additional variability in download rates (e.g., due to the difference in cache hits/misses) we pay particular attention to the impact of buffer-size dynamics.

To provide insights into the default performance of the SMP 2.0 player and further motivate its use in experimental studies, we first present results using SMP 1.6 and SMP 2.0. Figure 4.3 shows the player performance for 


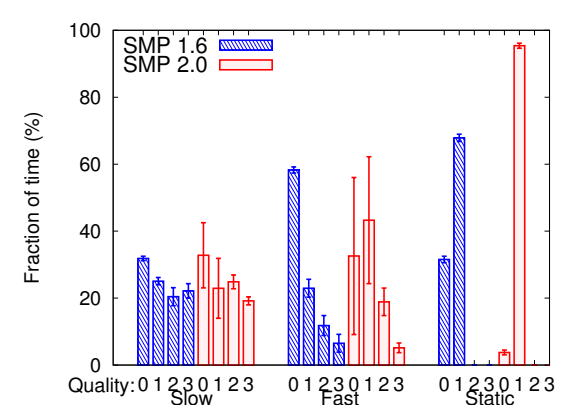

(a) Quality level

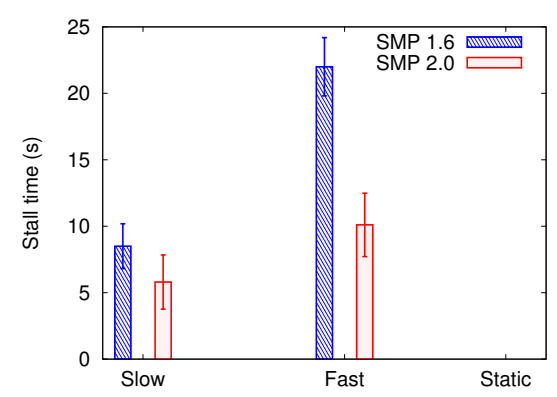

(c) Stall times

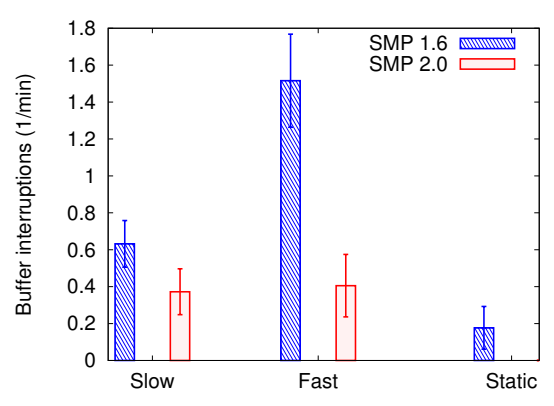

(b) Buffer interruptions

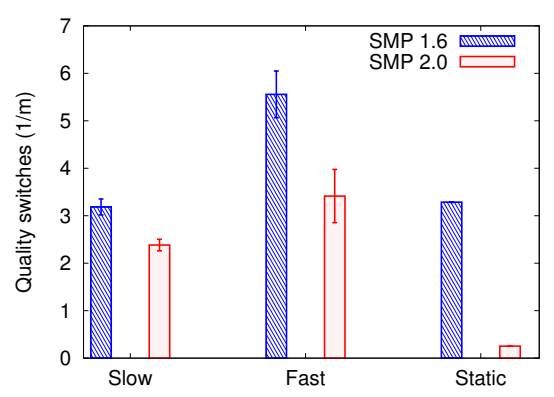

(d) Quality switches

Figure 4.3: Player performance comparison of SMP 1.6 and 2.0 under the three synthetic baseline scenarios.

the two players under each of the three synthetic workloads, each capturing a different degree of bandwidth variation. We observe that SMP 2.0 shows substantial improvements over the old SMP 1.6. In fact, it is noteworthy that for all three scenarios the new version outperforms the old player according to all four metrics. For example, in the static bandwidth case, SMP 2.0 quickly finds the maximum sustainable video quality and maintains that quality for the rest of the session. More than $95 \%$ of the time is spent in this state (Figure 4.3(a)). While omitted due to space constraints, similar results have been observed for our other scenarios, loss rates, and end-to-end delays.

Relative to the proprietary Netflix and MSS players, the SMP media player has a much smaller buffer, hinting that it is designed for shorter video playback. While the small buffer comfortably can accommodate for some fluctuations in the available bandwidth, during long periods of high bandwidth variability, the new player may still yield unsatisfactory performance. In general, it appears that SMP is dimensioned based on (relatively) static bandwidth scenarios, for which it is able to quickly rise to the highest 


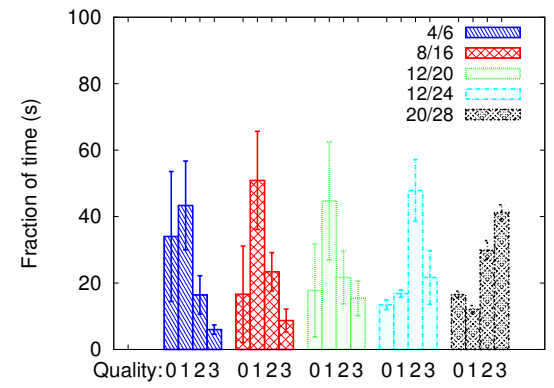

(a) Quality

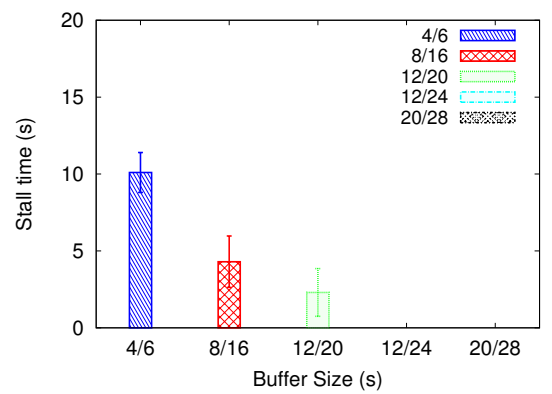

(b) Stall time

Figure 4.4: Performance impact of buffer sizes using the fast varying synthetic bandwidth trace.

possible encoding and maintain that quality. The smaller buffer footprint may also have advantages for legacy devices.

To obtain a better understanding of the impact of buffer-size dynamics, we modified the player so that it could be run with different buffer size configurations. ${ }^{1}$ In addition to the default buffer configuration $T_{\text {min }}^{\text {buf }} / T_{\max }^{\text {buf }}$ $=4 / 6$, we also used: $8 / 16,12 / 20,12 / 24,20 / 28$.

Figure 4.4 shows the observed video quality and stall times for the fast varying bandwidth scenario with a RTT of $50 \mathrm{~ms}$. The figure clearly shows that increasing the buffer size has positive effects on both the video quality being played and the stall times. For example, we are able to play with zero stalls for buffer sizes as small as $12 / 24$. With larger buffer sizes (e.g., at $20 / 28$ ), aided by long-term buffering effects, we are even able to play at the highest quality for more than $25 \%$ of time, despite the available bandwidth only exceeding the highest encoding rate for $25 \%$ of the time.

Figures 4.5 and 4.6(a) show the observed video quality and stall times for different buffer sizes, when running experiments using the real-world traces to emulate the available network bandwidth. For three of the real-world scenarios, we observe highly positive effects using larger buffer values. For example, the 20/28 configuration is able to eliminate stall times and allow high overall quality encodings for the bus, metro, and tram traces. It should be noted that for the metro and tram traces, this is achieved despite the available bandwidth not being sufficient to achieve the highest quality level for much of the trace duration. For these scenarios, Figures 4.4(a) and 4.6 suggest that there may be an inverse relationship between the buffer size and stall times, with much of the improvements related to $T_{m i n}^{b u f}$, which determines when the player resumes/starts playback. In contrast, in the

\footnotetext{
${ }^{1}$ To allow proper buffer management both buffer management and rate adaptation mechanisms had to be modified to account for the new buffer thresholds. In general, we tried to keep these modifications to a minimum.
} 


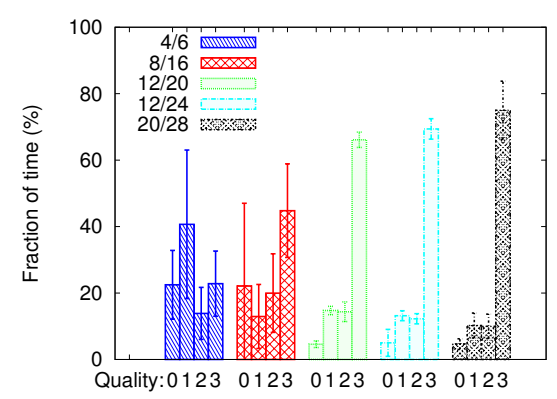

(a) Bus

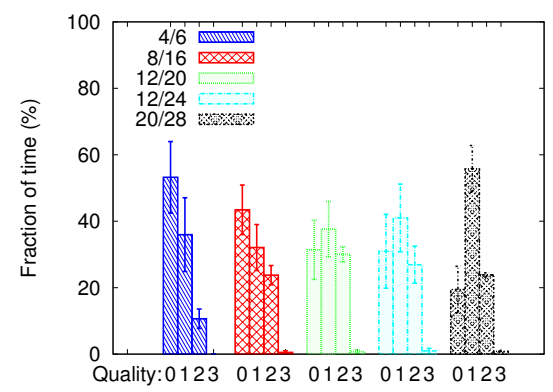

(c) Metro

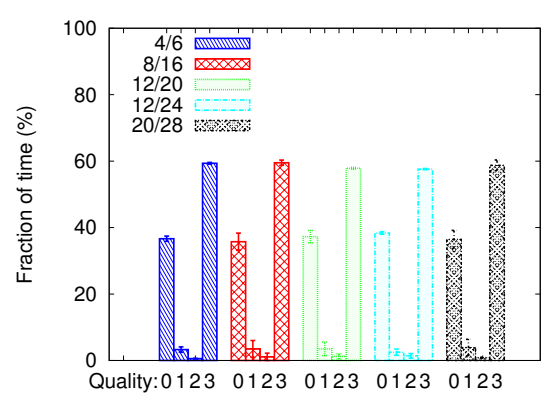

(b) Ferry

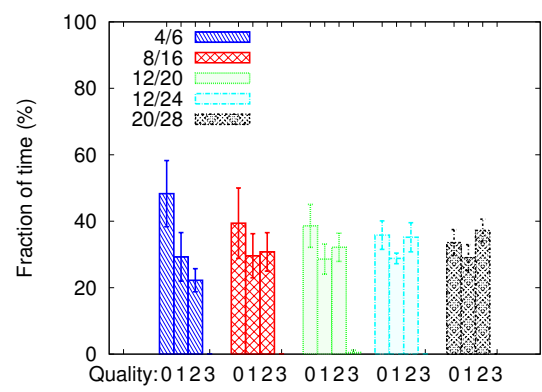

(d) Tram

Figure 4.5: Video quality under real-world scenarios for different buffer sizes.

ferry scenario, the larger buffer sizes provide little or no benefit. This is explained by the extreme workloads conditions during this trace: either very high bandwidth or very low bandwidth. For completeness, Figure 4.6(b) shows the corresponding startup delays.

\subsection{Longitudinal proxy performance}

This section describes a performance evaluation aimed at providing insights into the impact proxies and their policies may have on HAS performance. While caches and middle boxes already are widely deployed across the Internet, their impact on chunk-based content delivery, as exemplified here by $\mathrm{HAS}$, can also give hints on the design of future content-centric architectures [90]. For this reason, we consider a wide range of policies, including both policies that can be implemented in existing standard proxies, as well as more advanced policies that take into account the structure of the content itself. For our experiments we implemented and tested our policies in Squid (version 2.7-stable9), a popular open source cache that allows us to create our own caching and prefetching rules. 


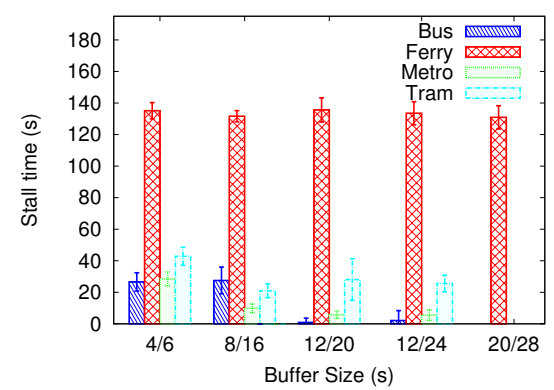

(a) Stall times



(b) Startup delays

Figure 4.6: Stall times and startup delays under real-world scenarios for different buffer sizes.

\subsubsection{Baseline policies}

Before designing advanced proxy-assisted policies, it is important to understand the performance impact of proxies and their policies under the most basic conditions. We first define a set of baseline policies which captures the basic performance tradeoffs seen in existing systems, and give some insights to how much room for improvement there may be.

- Empty cache: To capture the performance penalty associated with cache misses, we consider a policy in which the cache is cleared for each new client. This would correspond to the performance seen by the first requesting client, including clients that are requesting contents from the long tail of one-timers $[125,126]$. In this case, every chunk that the player requests will be fetched from the server.

- Full cache (all versions): To capture the performance under ideal proxy conditions we also include a policy in which the cache has been preloaded with all versions of every chunk. In this case, there will be no cache misses and the client can always be served from the cache, resulting in minimum transfer times.

- Best effort: To capture the performance of a standard proxy cache, that has seen a limited number of previous client requests, we use a simple policy that has cached every chunk that previously has been requested. In our experiments we start with an empty cache, pick a large cache size such as to avoid any cache replacement, and run the experiments for a sequence of clients.

While the first two policies correspond to the case of minimal and maximum cache hit rates, respectively, the third best-effort policy, captures the more realistic case of intermediate cache hit rates. On average, the cache 
hits will typically increase as more clients will have requested the same contents in the past, potentially reducing the transfer delays and increasing the TCP throughput for these clients.

For the best-effort policy we initialize all our experiments with an empty proxy cache, and allow one client to access the content at a time. This captures the performance observed by the $n^{\text {th }}$ client arriving to the system, assuming that there have been $(n-1)$ previous clients accessing the content and the cache does not remove any of the content that has been accessed in the past. In practice, a cache replacement policy, such as Least Recently Used (LRU), would typically be used. To interpret the results in this context, we note with the LRU policy there exists a time threshold (unique for each time instance) such that all chunks that were requested after this time are in the cache, and none of the other chunks are in the cache. Based on this observation, we can interpret the above abstraction as a cache operating under the LRU policy for which the current time threshold (of what is in the cache) is such that the content requested by the last $(n-1)$ clients are in the cache, but no other chunks. Having said this, it is important to note that this typically would result in a conservative estimate, as the average cache hit rate that a LRU system in steady state likely would require slightly less storage. In practice, such a system would likely see more frequent (and similar) accesses to low quality contents, which may be frequently accessed during the transient time period during which the cache is being filled.

\subsubsection{Quality and content-aware prefetching}

We next describe three basic content-aware prefetching policies that take into account the content structure, as well as the quality level that is currently being requested.

- 1-ahead: In the most basic prefetching policy, the proxy fetches the next chunk with the same quality as the chunk that is currently being downloaded. When the client has fairly stable bandwidth this policy should yield higher cache hit rates. The policy may waste bandwidth whenever the client switches quality.

- $n$-ahead: A somewhat more aggressive policy is to prefetch up to $n$ chunks ahead of the currently requested chunk, but still only prefetching chunks of the same quality level as the currently requested chunk. Under scenarios with many quality switches this policy can result in much wasted bandwidth between the proxy and server.

- Priority-based: To take advantage of the case when the cache already has the content that may be the most natural to prefetch next, we present a priority-based policy that (i) only prefetches one chunk ahead, but (ii) takes into account both the current cache content and the status of the client (as seen from the perspective of the proxy) 
when selecting what quality this chunk should have. In the case that the client last switched to a higher encoding and it is not the first time that the client is requesting chunks of this video quality (i.e., at some earlier time it has switched down from this quality), we use the following priority: (i) current quality, (ii) one quality level below, (iii) one quality level above, and (iv) no prefetching. In all other cases (i.e., the client last switched to a lower quality or has never been at this quality level before), we use the following priority: (i) current quality, (ii) one quality level above, (iii) one quality level below, and (iv) no prefetching.

When interpreting the priority-based policy, it is important to note that the player always starts at the lowest level and initially is trying to ramp up to as high a quality level as possible. After it has reached a sustainable quality level it would typically oscillate between two neighboring quality levels. In contrast to the two first policies, the priority-based policy uses spare bandwidth capacity (due to previously downloaded chunks) to guard against future quality switches.

\subsubsection{Client-proxy cooperation}

Perhaps one of the main problems with the basic cache policies and contentaware prefetching policies is that the quality selection choices made by the clients are independent of the content on the proxy. For example, in a scenario in which a client is quickly obtaining chunks cached by the proxy, the client may be tempted to increase the quality it is requesting, even in cases where the proxy does not have the higher encoding and there may not be sufficient bandwidth between the proxy and the server to quickly obtain this content.

In this section we present a cooperative policy class that allows chunk selection and prefetching decisions to be made based on information shared between the proxy and the client. By cooperation, the goal is to improve both the hit rates at the proxy cache and the viewer quality at the client.

Consider a system in which the client continually shares its buffer occupancy with the proxy and the proxy shares the map of chunks that it has access to with the client (for the particular file that the client currently is downloading). This information can (i) be shared at the beginning of the download and then updated as the cache content changes, or (ii) the proxy can continually update the client about the cache content for all chunks belonging to some upcoming time window from the current download point. In either case the client can give preference to downloading chunks that the proxy has. Among the chunk qualities stored on the proxy, we assume that the client would select the chunk quality with the most similar quality to that suggested by its rate-adaptation scheme (giving strict preference to lower qualities, if the intended quality is not available). ${ }^{2}$ Now, given the

\footnotetext{
${ }^{2}$ Policies that request the chunks stored at the proxy with a probability $p$, and other-
} 
clients request we consider two policy versions for the prefetching actions taken by the proxy.

- Buffer oblivious: This policy does not consider the client buffer state. Instead, it blindly uses the priority-based prefetching policy from the previous section.

- Buffer aware: This policy consider the client's current buffer occupancy. If the buffer occupancy is below a threshold $T \leq T_{\min }^{b u f}$ we use the following prioritization order: (i) current quality, (ii) one quality level below, (iii) two quality levels below, and (iv) no prefetching. On the other hand, if the client has higher buffer occupancy, we use the standard priority-based prefetching policy.

For the purpose of our performance evaluation, we developed a proof of concept implementation of the above policies. For this we had two options. We could either modify the player's source code, or build a wrapper around the player which intercepts and modifies the client requests. Due to a number of constraints with the flexibility of the SMP software, we selected the second approach, and make use of Tcpcatcher ${ }^{3}$, a proxy based monitoring software which provides handles to sniff and change packet contents on the fly.

At a high-level, the client connects to the Tcpcatcher port, and this port connects to the child port of our modified Squid proxy. This allows us to modify the client requests on the fly, such as to take into account the proxy contents. The client software is modified to send the buffer occupancy once a second, and the proxy sends the required chunk information to our Tcpcatcher module, which makes the decisions on behalf of the client. While our solution is non-optimal, in that SMP 2.0 is oblivious to any alterations to its requests, and a production implementation that modifies the player code could do additional enhancements to the rate adaption algorithm so as to achieve finer grained control, we do not expect any major performance differences with such implementations.

\subsubsection{Performance evaluation}

We now present the results of the experiments with different proxy cache configurations and policies. Similar to for the non-proxy case, we have found that our conclusions do not appear to depend on the loss rate and round-trip times. Instead, the major factor is the bandwidth variations and which link is the bottleneck. Due to these reasons and to save space, in the following, we present results only for the case in which the proxy-server RTT is $50 \mathrm{~ms}$, the client-proxy RTT is $6 \mathrm{~ms}$, and client uses the default buffer configuration $T_{\min }^{\text {buf }} / T_{\max }^{\text {buf }}=4 / 6$.

wise (with a probability $(1-p)$ ) pick whatever chunk is selected by its rate-adaptation mechanism are of course also possible.

${ }^{3}$ TcpCatcher. http://www.tcpcatcher.fr/, Sept. 2012 


\section{Client-proxy bottleneck}

Consider first the case when the bottleneck is between the client and proxy. For this case, we show results for when the proxy-server connection is not constrained, but the available client-proxy bandwidth follows the synthetic scenario with fast bandwidth variations.

To allow a fair comparison between policies, we replay the synthetic traces such that each of the policies sees exactly the same overall bandwidth conditions and clients arrive at the same time instances in each experiment. At the same time we make sure that each of the ten clients accessing the video, see different bandwidth conditions at each time instance of their individual download sessions. This way, the $n^{\text {th }}$ client in each experiment sees exactly the same bandwidth conditions, but different than the $m^{\text {th }}$ client, where $m \neq n$.

Figure 4.7 shows the averaged viewer quality and stall times over an experiment with ten clients for each of the six policies defined in Sections 4.5.1 and 4.5.2. Note that despite improvements, the performance differences between the full cache policy (best case) and empty cache policy (worst case) are relatively small. ${ }^{4}$ This suggests that proxies, regardless of policy, can provide only very limited performance advantages in the case the clientproxy link is the bottleneck.

Having said this, it should be noted that the simple 1-ahead prefetching policy is able to achieve most of these advantages. In contrast, the basic best effort policy performs very similar to the empty cache case. Our results also suggest that while there are performance improvements to prefetching, there is little gain to excessive prefetching. In fact, some clients experienced performance drops due to excessive prefetching clogging up the TCP pipeline.

To illustrate the longitudinal quality benefits of cache assistance for this scenario, Figure 4.8 shows the observed quality as a function of the number of previous clients that has downloaded the content when using the 1-ahead prefetching policy. The results here are the average statistics over five runs, each with ten clients. While the high variability in quality that different clients observe makes it more difficult to compare and distinguish the absolute quality levels observed by each client, it is clear that there is a longitudinal quality improvement benefiting clients arriving later.

We next consider the cache hit rate of the different policies. Figure $4.9 \mathrm{a}$ shows the cache hit rate as a function of the number of previous clients that have downloaded the video. Note that after two-three clients most policies

\footnotetext{
${ }^{4}$ To make sure that the small performance improvements were not due to optimizations made at the server we took a closer look at the service times of individual chunks, when served by the server or the (full) proxy cache respectively. In fact, for the case with the same RTT to both the server and the proxy, we observed a smaller time to first byte $(\bar{x}=$ $8.30 \mathrm{~ms} ; \sigma=15.03 \mathrm{~ms})$ for the full cache than for the server $(\bar{x}=16.10 \mathrm{~ms} ; \sigma=7.85 \mathrm{~ms})$. For the time to last byte, the differences were negligible: full cache $(\bar{x}=2.71 s ; \sigma=1.92 s)$ and server $(\bar{x}=2.81 s ; \sigma=1.97 s)$.
} 


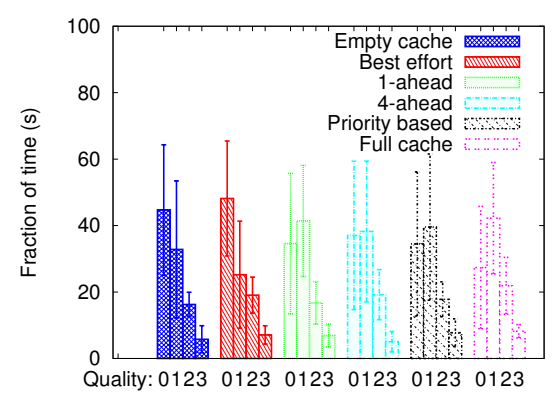

(a) Quality level

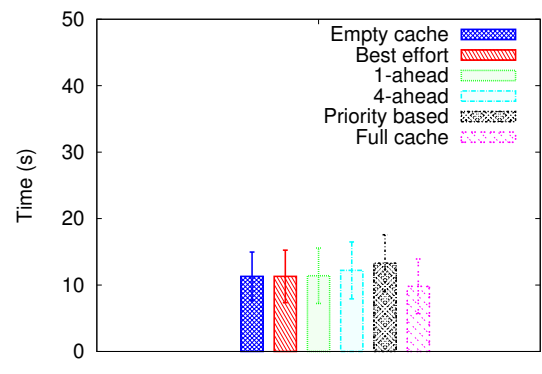

(b) Stall time

Figure 4.7: Comparison between baseline and content-aware proxy policies, and the bottleneck is between the clients and proxy.

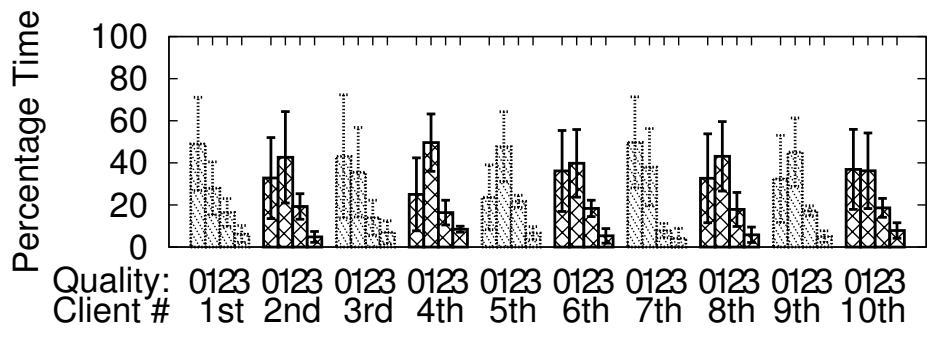

Figure 4.8: Observed quality levels over $n$ subsequent client downloads when using 1-ahead prefetching with client-proxy bottleneck.

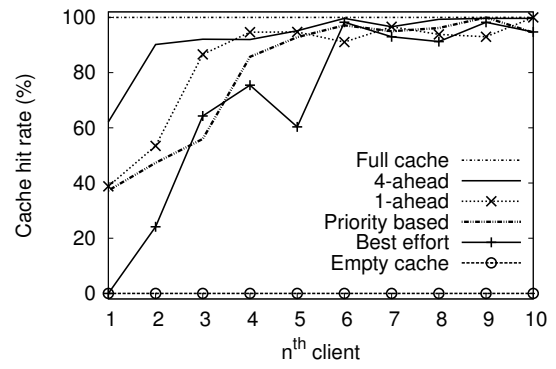

(a) Client-proxy

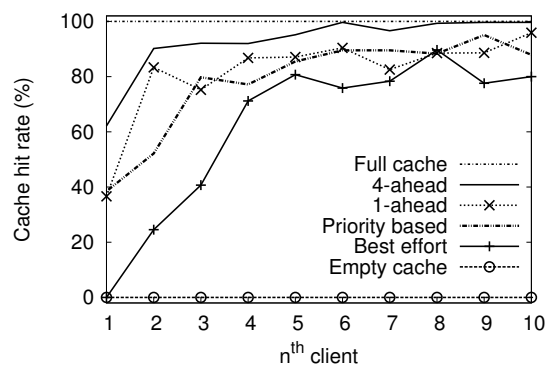

(b) Proxy-server

Figure 4.9: Hit rate as a function of the number of previous downloads, when either client-proxy or proxy-server bottleneck.

have a hit rate of $80-90 \%$. For the best effort policy it takes roughly five clients before the hit rate surpass $90 \%$. 
Table 4.1: Bandwidth usage between proxy and server for the policies defined in Sections 4.5.1 and 4.5.2.

\begin{tabular}{|l|c|c|}
\hline & \multicolumn{2}{|c|}{ Bandwidth bottleneck location } \\
\hline Policy & Client-proxy & Proxy-server \\
\hline Empty cache & $169.2 \mathrm{Mb}$ & $184.9 \mathrm{Mb}$ \\
Best effort & $59.5 \mathrm{Mb}$ & $94.7 \mathrm{Mb}$ \\
1-ahead & $64.9 \mathrm{Mb}$ & $65.9 \mathrm{Mb}$ \\
4-ahead & $77.09 \mathrm{Mb}$ & $77.85 \mathrm{Mb}$ \\
Priority & $81.3 \mathrm{Mb}$ & $72.2 \mathrm{Mb}$ \\
Full cache & - & - \\
\hline Cooperation/oblivious & $89.97 \mathrm{Mb}$ & $97.2 \mathrm{Mb}$ \\
Cooperation/aware & $103.7 \mathrm{Mb}$ & $105.77 \mathrm{Mb}$ \\
\hline
\end{tabular}

When interpreting the somewhat higher overall hit rates observed by the prefetching policies, it should be noted that there may be some bandwidth wasted when chunks are prefetched. The first six rows of the left-hand column of Table 4.1 summarize the bandwidth usage (measured as the total transferred bytes) between the proxy and the server for these experiments. We see that both the best effort $(59.5 \mathrm{Mb})$ and 1-ahead $(64.9 \mathrm{Mb})$ achieve similar bandwidth usage savings compared to the empty cache policy (169.2 $\mathrm{Mb}$ ), while in both cases delivering the clients higher quality encoding. This suggests that the extra chunks obtained through prefetching often may be useful for later requests (as seen by the higher hit rates).

\section{Proxy-server bottleneck}

We next consider the case when the bottleneck is between the proxy and the server. We show results for when the client-proxy connection is not constrained, but the available bandwidth of the proxy-server link follows that of the synthetic scenario with fast bandwidth variations.

Figure 4.10 shows the averaged viewer quality and stall times for each of the six policies defined in Sections 4.5.1 and 4.5.2. Comparing the full cache policy and empty cache policy, we note that there is very large performance potential for proxy caching in this scenario. Overall, the full cache policy has significantly superior performance compared to all the other policies. It has no stall times and is able to serve the clients almost entirely at the highest quality.

It is encouraging that a significant performance improvement is achieved with the best effort policy alone. This policy is easy to implement and does not require content awareness. Yet, it is able to reduce the stall times and allow the video to be played at a much higher quality than with the empty cache policy (or no cache, for that matter).

It may, however, be discouraging that none of the three basic prefetching 


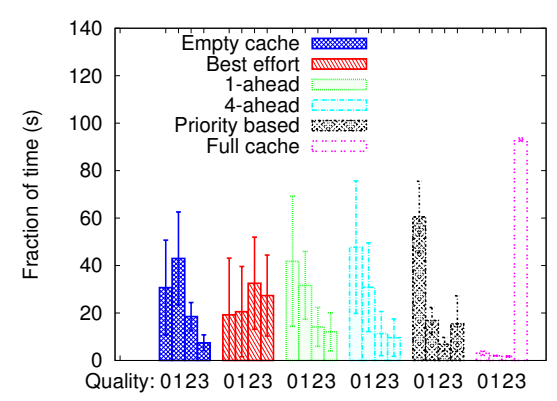

(a) Quality level

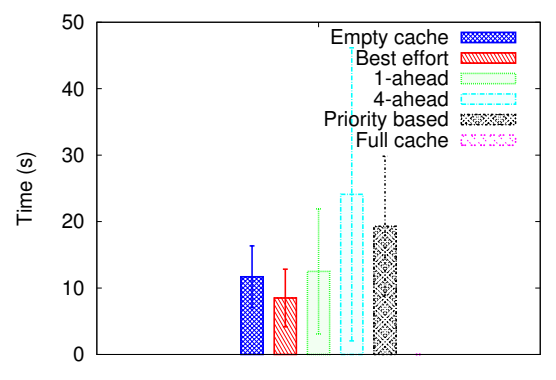

(b) Stall time

Figure 4.10: Comparison between baseline and content-aware policies; proxy-server bottleneck.

policies are able to improve on this. In fact, rather the opposite, these policies all achieve worse performance than the best effort policy. The problem with these policies is that the prefetching itself clogs up the bottleneck between the proxy and server. This illustrate that more careful policies, such as our cooperative policy, are required to take better advantage of the cache and its bandwidth.

The limited success of the prefetching policies can be further observed when considering the cache hit rates. Figure $4.9 \mathrm{~b}$ shows the hit rate as a function of the number of previous clients. Comparing with Figure 4.9a, we note that the hit rate in this scenario is significantly lower than the hit rate seen when the bottleneck is between the client and proxy.

Due to space constraints we can only include a limited number of representative results for our default scenario. Table 4.2 summarizes the average playback quality for the corresponding experiments for the real world scenarios and experiments with larger loss rates and RTTs. In general, we have found that our conclusions hold true also for these scenarios.

\section{Client-proxy cooperation}

Motivated by the large performance gap between the full cache and best effort policy, we consider the performance under the client-proxy cooperative policies. Ideally, these policies should help improve performance by adjusting the requested quality level based on the content in the cache.

Figures 4.11(a) and 4.11(b) show the quality levels for these policies for the cases when the client-proxy link is the bottleneck and when the bottleneck is between the proxy and the server, respectively. The stall times are similar to those of the 1-ahead policy. Comparing these results with those in Figures 4.7 and 4.10, note that the cooperative policies can significantly improve the viewed video quality. Not surprisingly, the largest improvements are achieved when the bottleneck is between the proxy and server. In 
Table 4.2: Average playback quality (measured in Kbps) of example policies under different scenarios.

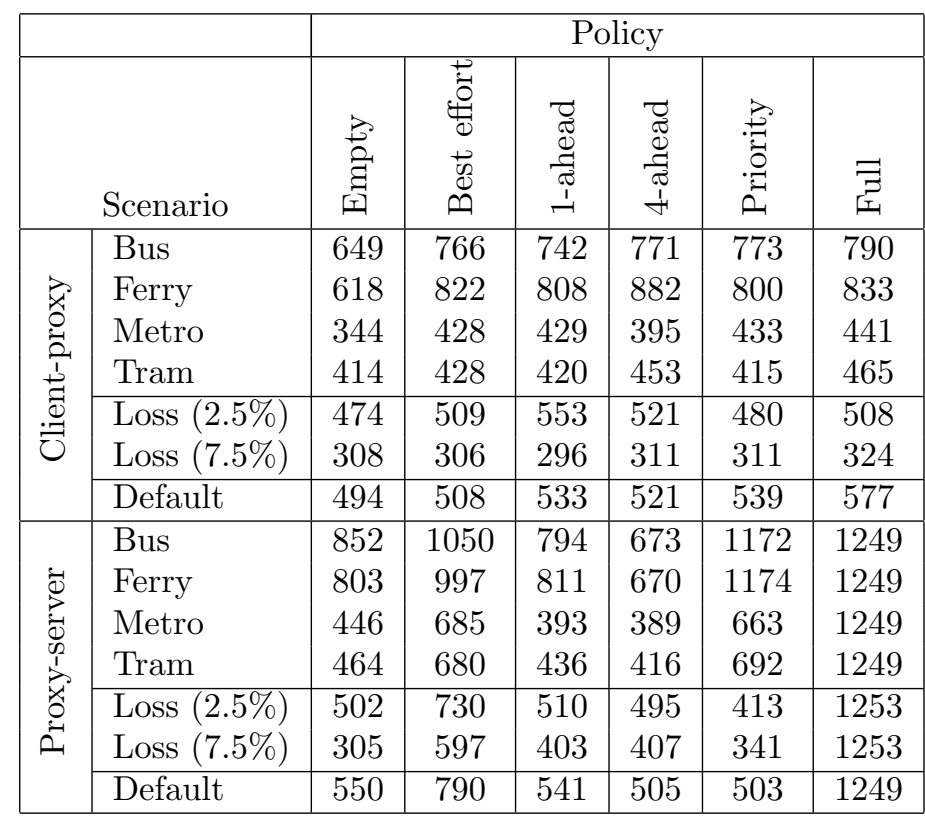

this case the policies are able to effectively leverage the spare proxy-server bandwidth enabled by the boosted hit rates, to prefetch chunks that are likely to help the current or future clients.

The high playback quality achieved by the buffer oblivious cooperative policy is particularly impressive for the case the bottleneck is located between the proxy and server. For this case, as shown in Table 4.1, the buffer oblivious policy uses almost the same bandwidth $(97.2 \mathrm{Mb})$ between the proxy and server as the best effort policy (94.7 Mb), but delivers much higher quality to the client.

To illustrate the performance improvements observed by later clients, Figure 4.12 shows the observed quality as a function of the number of previous clients. While this data is noisy due to the difference in bandwidth observed by each client, it is clear that the cooperative policy is able to load the cache with content that future clients can benefit from, without hurting the performance of the early clients.

\section{Buffer size}

For our final experiments, we revisit the question about how the player buffer size may impact our conclusions regarding which policies are the most advantageous in each scenario. In general, and as shown in Section 4.4, we 


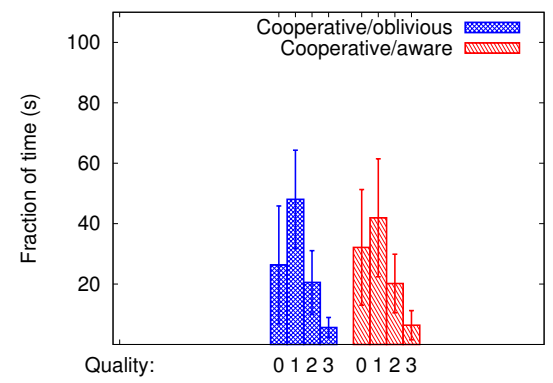

(a) Client-proxy

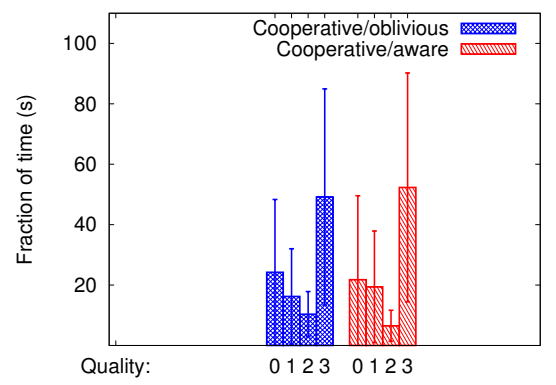

(b) Proxy-server

Figure 4.11: Client-proxy cooperation experiments, when client-proxy and proxy-server bottleneck.

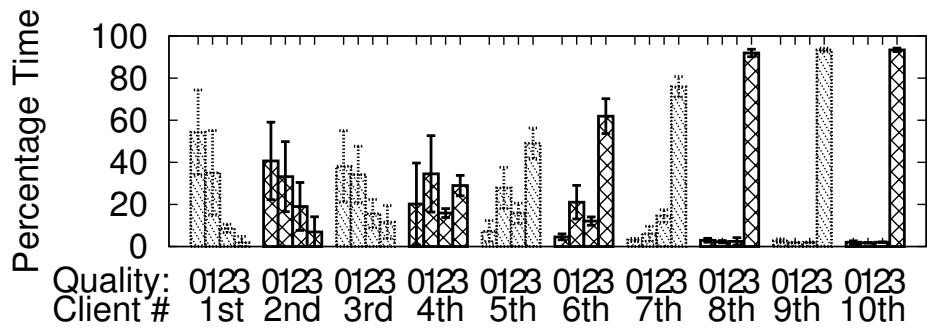

Figure 4.12: Observed quality levels over $n$ subsequent client downloads when the cooperative buffer oblivious policy is used, and the bottleneck is between the proxy and server.

have found that a larger buffer size reduces the amount of buffer interruptions, increases the average video quality, and helps improve the overall video playback experience.

Figures 4.13 and 4.14 summarize the video quality and stall times, respectively, when $T_{\text {min }}^{\text {buf }} / T_{\text {max }}^{b u f}=12 / 20$ (rather than the default buffer size of $4 / 6$ ) for the cases when the bandwidth bottleneck is between the clientserver and proxy-server. In addition to overall better performance, it is interesting to note that the simpler best effort policy benefits the most from the larger buffers. The best effort policy is able to achieve very small stall times and relatively high video quality. In fact, in many cases it outperforms the more complex prefetching policies. These results suggest that a larger client buffer reduces the importance of advanced proxy policies. With larger client buffers resulting in unnecessary data transfers when users terminate their viewing early, advanced policies may therefore be most attractive in wireless environments, with relatively expensive links with battery-powered mobile devices.

The much shorter overall stall times for the best effort policy are due to 


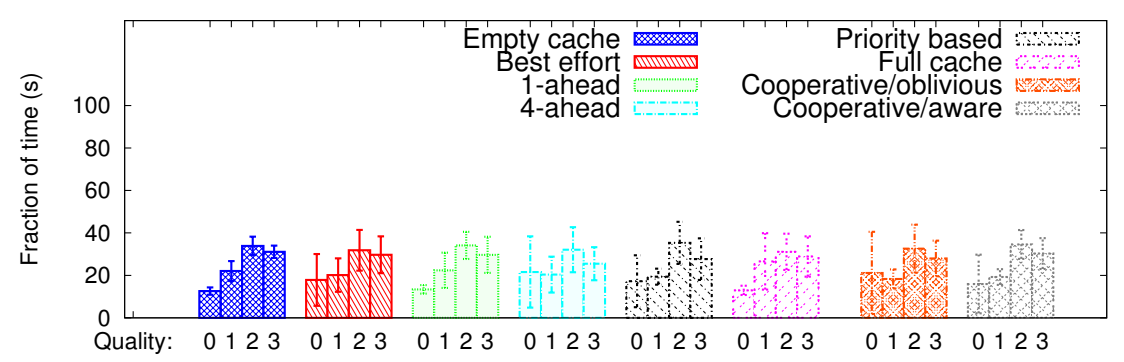

(a) Client-proxy

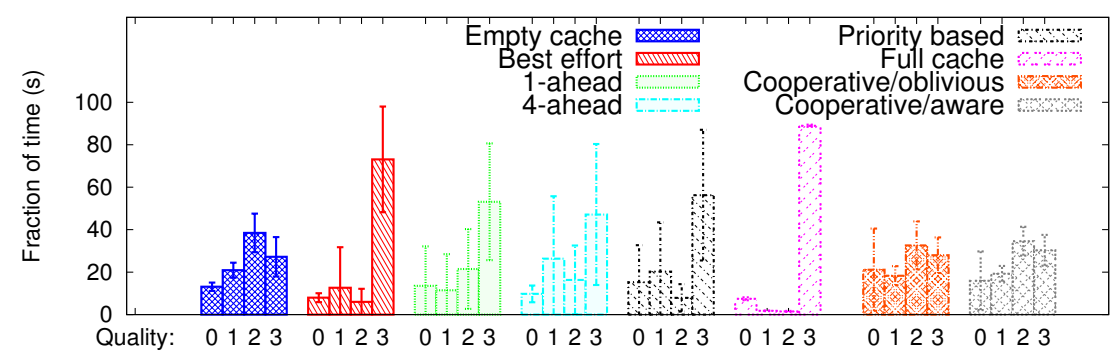

(b) Proxy-server

Figure 4.13: Summary of quality level statistics under different policies when using larger client buffer.

two compounding factors. First, the best effort policy sees a much bigger reduction in the number of stalls per minute (by a factor of 8.4 compared to 2.6 for the 1-ahead policy, for example) and in the average stall time per playback interruption (by a factor 2.0 while the 1-ahead policy increases by 1.3). These two factors have a multiplicative effect, resulting in much smaller overall stall times.

\subsection{Conclusion}

With HAS responsible for large traffic volumes, network and service providers may consider integrating customized HAS-aware proxy policies. In this chapter we consider the potential performance impact of such optimizations.

We present an experimental framework using open source Squid proxies and the most recent Open Source Media Framework (OSMF). The open source software allows us to implement and test content-aware policies, while measuring the performance as seen at the client.

Using this framework, we first present a scenario-based performance evaluation of the latest version of the OSMF player, and show that this player has substantially improved performance in comparison to the versions employed in previous work. We then present a thorough longitudinal evalu- 


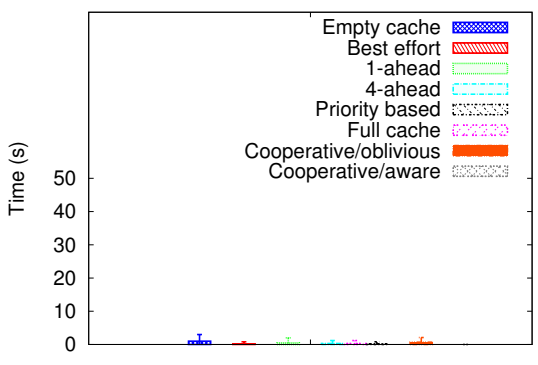

(a) Client-proxy

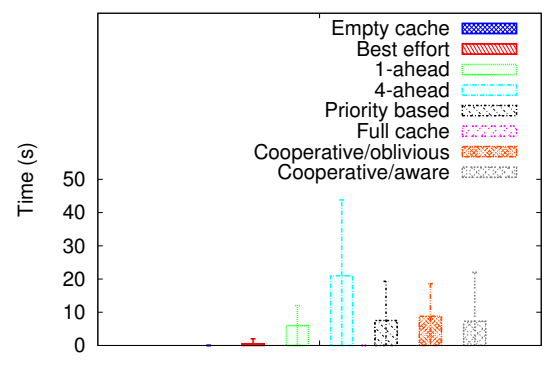

(b) Proxy-server

Figure 4.14: Summary of stall times under different policies when using larger client buffer.

ation in which we evaluate a range of different policy classes that may be employed in proxy-assisted systems and provide insights to the effectiveness of different policies and their performance tradeoffs. We quantify the benefits using different proxy-assisted solutions, including basic best effort policies and more advanced content quality aware prefetching policies. Finally, we present and evaluate a cooperative framework in which clients and proxies share information to improve performance. Our results show that the bottleneck location and network conditions play central roles in which policy choices are most advantageous, and that careful proxy design and policy selection is important when trying to enhance HAS performance in edge networks.

Future work includes the development and testing of adaptive proxy policies that make prefetching decisions based on estimates of the available bandwidth for both the client-proxy and proxy-server connections. Such policies could, for example, perform more aggressive prefetching during times when there is spare bandwidth between the proxy and server. 


\section{Chapter 5}

\section{Prototype implementation of interactive branched video player over HAS}

\section{$5.1 \quad$ Introduction}

The Internet and the World-wide Web is continually transforming the way people access information and content. Today, many people use the Internet for their daily entertainment, and on-demand media is playing an increasingly central role in our lives. This trend is particularly apparent when considering the current volume of video traffic [127] and the quickly growing content catalog offered to online users. For example, more than 72 hours of new content is uploaded to YouTube each minute. ${ }^{1}$

\subsubsection{Personalized interactive branched video}

In contrast to traditional $\mathrm{TV} /$ radio broadcasts, Web-based services often operate on an on-demand basis and only serve clients when explicit requests for service are made. In addition to full length movies offered by popular on-demand services such as Netflix, much of the traffic is for user generated content (UGC). The rapidly increasing movie catalogs of these sites provide users with an enormous amount of contents for all tastes and interests. The success of sites such as YouTube also illustrate that there is great value in leveraging the creative power of regular home users. One contribution of this chapter is that we enable a solution that further empowers the creative home user, such that they easily can define personalized interactive branched

\footnotetext{
${ }^{1}$ YouTube statistics, www.youtube.com/yt/press/statistics.html, March 2013.
} 
video, based on any previously created video(s).

In contrast to a conventional video, which consists of a single sequence of video frames that are viewed sequentially from a chosen starting point, interactive branched video consists of multiple alternative plot sequences seamlessly linked, allowing multiple possible playback paths differing in the media portions they include and/or in their ordering. Perhaps the simplest generalization of a traditional (linear) movie to an interactive branched video is a movie in which the viewer is able to choose among a variety of possible endings and/or plot sequences. There are already DVD releases of movies with multiple endings (e.g., the DVD edition of the Hollywood movie "Clue"), and it is easy to imagine generalizations to more general tree or graph structures.

In this work, we present a generalized form of interactive branched video. First, the creator can present the user with multiple alternative sub-plot options as the user is traversing the media. The path choices can be selected online (while viewing the media) either by the user, or based on information about the user, including the user's previous path choices. Second, each such path segment can be defined as a customized alternative sub-plot, without having to make any changes to the underlying (linear) media object. Our solution allows the creator to define any arbitrary set of paths and nonlinear sequences through the media.

\subsubsection{HTTP-based adaptive streaming}

There is an increasing demand for personalized delivery to increasingly heterogeneous users (e.g., using mobile devices). To better utilize the available bandwidth and improve the service of each client, many content providers (including Netflix) have begun using HTTP-based Adaptive Streaming (HAS) [25, 64,120]. In contrast to basic HTTP-based streaming, with HAS the video is encoded into different qualities and the player, at each point in time, adaptively chooses the most suitable encoding based on the current buffer and network conditions. While HAS allows the service quality to be adapted to the individual user's device and network conditions, it does not customize the content itself.

In this chapter we introduce the concept of HAS-based interactive branched streaming, effectively enabling customization of the content itself. The use of HAS also has the advantage that the different video sequences are easily addressable by the player and the quality of each path can be adapted to match the available bandwidth.

\subsubsection{Design contributions}

This chapter presents the design, implementation, and validation of the functionality of our HAS-based interactive branched streaming solution. Our design has four novel components. 
- Light-weight personalizable metafile: To provide developers with maximum possible flexibility, we create a personalizable metafile that defines the interactive branched, and nonlinear structure, separate from the regular media object and the accompanying HAS metafile (that defines quality encodings etc. of the media itself). In this file, the developer can easily define alternative sub-plots (nonlinear sequences) than those defined by the underlying linear media, and provide the user with path choices between such alternative plot sequences.

- Longest-path matching: With focus on the path choices offered to users, the overall path structure is defined using branch-point rules. A creative developer may therefore define individualized branch-point rules that take into consideration past path choices made by the users. To break ties when there are multiple branch-point rules that match a particular user's current playback path, we employ a longest-path matching policy that always picks the rule that most closely specifies the conditions for when the rule should be applied.

- Branch-point-aware buffer management: To ensure seamless playback with minimum playback interruptions, while allowing the user to defer playback decisions as late as possible, careful buffer management and prefetching is required. For each branch point, the player prefetches chunks for the different candidate paths into a prefetch buffer and manages the content of the playback buffer as needed.

- Rate-adaptation with priorities: To maximize the expected playback quality without playback interruptions, the download rate is carefully estimated, the priority of the different path choices weighted, and the qualities of the prefetched chunks selected. While we leave a deeper evaluation for future work, we provide some promising validation results for one such candidate policy.

To the best of our knowledge, no prior work has combined adaptive streaming and interactive branched video to achieve seamless streaming of interactive branched video. We argue that merging these two ideas is natural and allows a higher degree of personalization for heterogeneous clients than is feasible with regular video.

Using a relatively simple metafile structure, a creator can easily define any alternative plot sequences, assign path priorities, and present the user with path selections. Our generalized and novel interactive branched video format provides a high degree of flexibility for the creative media developer. The flexibility coupled with seamlessly linked media sequences is attractive when offering personalized or customizable versions of many existing services (such as news on-demand, virtual tours, etc.). However, perhaps more importantly, the simplicity of our solution opens the door for user-generated interactive branched nonlinear video; creating an endless possibility of new opportunities and media forms. 


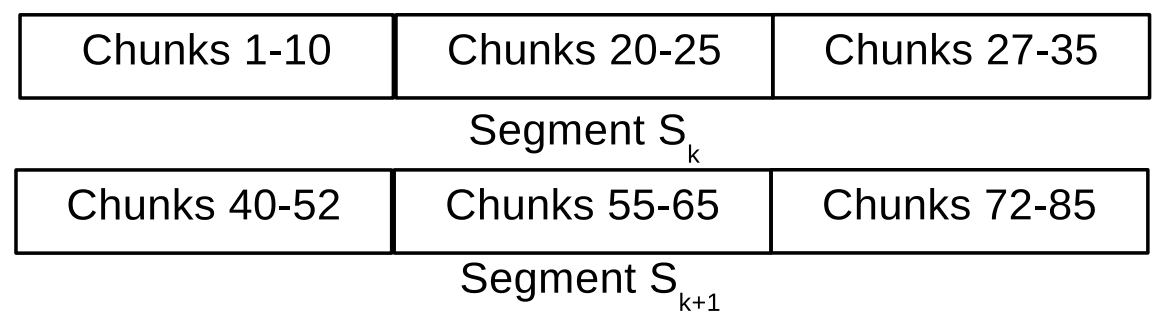

(a) Nonlinear segments
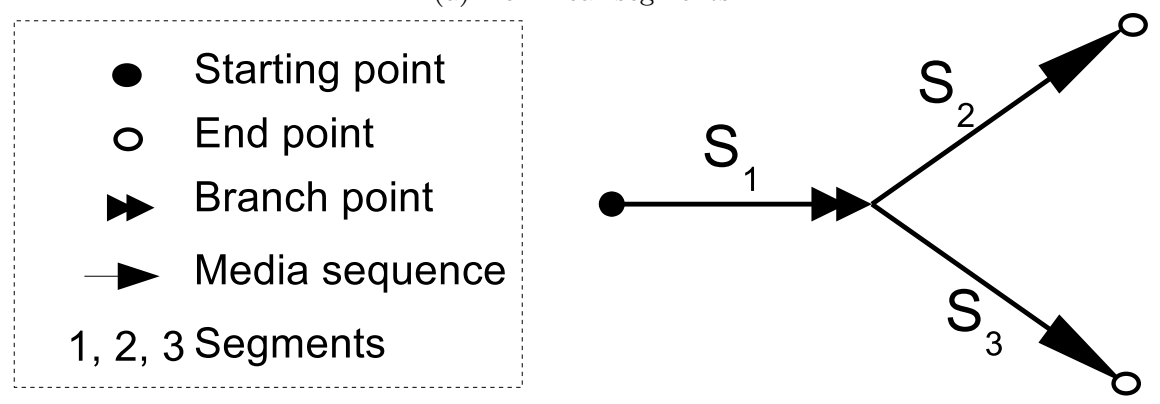

(b) Interactive branched video

Figure 5.1: Example media structure.

While our solution applies to any HAS-based media, our proof-of-concept implementation is implemented as an extension to Adobe's Open Source Media Framework (OSMF). Validation is performed under a range of network conditions and example structures. Our results are encouraging, and we believe that the simplicity of our solution will allow for easy creation of user-generated interactive branched videos, and countless possibilities for the creative media designer.

\subsection{Interactive branched, nonlinear video}

Before going into the details of our solution, we first define our terminology. In contrast, to previous works (e.g., $[103,106]$ ), which typically use the term nonlinear media to refer to media in which the user can take different paths, we build our terminology around the playback patterns based on the original linear media object.

- Chunks and segments: With HAS, the video is downloaded in units a few seconds long that we term chunks. We term a sequence of chunks a segment. Note that some authors use the term segment for what we call a chunk.

- Interactive branched video: Any video in which users may take different predefined paths through the media. In most cases, such video 
can be described using a directional graph structure in which each edge corresponds to a (nonlinear) segment, and each vertex with two or more outgoing edges corresponds to a path choice. Figures 5.1(b) and 5.3 provide two such examples.

- Nonlinear segment: A playback sequence of chunks stitched together into an alternative sub-plot than defined by any consecutive media sequence in the underlying (linear) media object. For example, in Figure 5.1(a) a nonlinear segment is defined which has cut out the chunks between 10 and 20, as well as between 25 and 27 .

We allow chunks to appear in multiple nonlinear segments, and segments can be present along many different paths. This interactive branched nonlinear video structure allows any arbitrary graph structure and path choices to be defined.

\subsection{System Design}

\subsubsection{Design goals}

When designing our interactive branched solution we had five primary design goals.

- Flexibility: The solution should provide developers with flexibility to define new creative media, without requiring any changes to the underlying linear media object. By giving developers full flexibility, we hope to maximize the creativity of future media developers, leaving room for an endless possibility of new media.

- Personalization: The solution should make it easy to personalize the viewer experience based on both information about the user and previous path choices made by the user.

- Privacy: Personalization of the viewer experience should be possible based on both information that the user is willing to share with the server (i.e., public information) and information that it is not willing to share with the server (i.e., private information).

- Light-weight traffic overhead: The solution should include tools to reduce the traffic overhead associated with both delivering the redundant file data, as well as any additional meta data associated with the interactive branched content itself.

- Adaptive and seamless playback: To ensure seamless playback and effective resource usage for heterogeneous clients, the solution should include prefetching, as well as quality-aware rate adaptation. 
In addition to the above primary goals, extensions are also possible which can enhance our solution. For example, to improve the user experience (e.g., as measured by the average playback quality) or the degree of personalization, additional optimizations at the server end are possible. One approach to achieve this is to leverage information obtained based on previous user interactions with the service, such as to modify the metafiles future clients are presented. This chapter focuses on the first four goals and presents a proof-of-concept validation of the fifth.

\subsubsection{System overview}

As summarized in Section 5.1.3, our design includes five novel components. Much of our design is centered on the manifest file of the original media, the interactive branched nonlinear metafile, and the information provided in these files. Our solution is client-driven, and clients simply download these files before beginning playback. In general, there are no restrictions on the server or manner these files are downloaded.

The addition of a separate light-weight personalizable metafile helps separate the information known by the server (original manifest) from that only known by the client. The metafile provides the user with the necessary information about the interactive branched nonlinear aspects of the media. The manifest file describes the underlying media object and the playback qualities in which each chunk can be downloaded.

During playback, our modified HAS player identifies the next branch point rule that the client will reach, prefetches media associated with each of the alternative path choices, performs branch-point-aware buffer management, and adapts the quality of the prefetched chunks based on the available bandwidth conditions, bandwidth needs, and the relative priority of each path choice. Overall, good prefetching and rate adaption policies must weigh high video playback quality against the risk of playback interruptions.

No server-side modifications are required; the server simply serves each chunk request.

\subsubsection{Metafile structure}

Our branched video metafile provides developers with maximum possible flexibility, and is designed to address the first four design goals of our system. The metafile allows the interactive branched structure to be defined using two components:

- Nonlinear segments: At the convenience of the creator, alternative sub-plots than those in the underlying linear media can be defined using nonlinear segments. These segments are defined as a list of linear chunk sequences, stitched together from the original video, and a shortcut label that allow easy referencing. Of course, this functionality 
could also be implemented as a sequence of branch points without alternative choices beyond the default path.

- Branch-point rules: The overall path structure is defined using branch-point rules, with each rule specifying: (i) the characteristics for the client for which the rule applies, (ii) the path sequences that the client must have taken for this branch point rule to apply, (iii) the available path choices at the branch-point, (iv) their respective priorities, and (v) the corresponding explanations to be presented to the user when selecting between the path choices.

Branch rules can be used in two ways: user-driven or knowledge-driven choices. The most obvious way is to present clients with path choices, define priorities with which the initial chunks for each of those path choices should be prefetched, and allow the client to pick among these path choices. The second way is for the player to make the decision for the user based on private information about the client or other internal knowledge about the client's previous path choices, for example, at the time the branch point is reached. Note that this information may not be known at the time the metafile is downloaded and/or created.

Our approach reduce the overhead at the media server and ensure that the server do not have to maintain individual copies of the content and/or maintain state information on a per-client basis during the playback itself. The separation between metafile and manifest file allow the client downloading the metafile from any Web server, and the media server must only serve request for individual chunks.

A common metafile can be created and shared with clients requesting access to the the service, or personalized metafiles can be created at the time of the client request. In both cases the clients independently request and download the parts of the original media content that matches its personalized and/or selected media paths.

\subsubsection{Longest-path matching}

Our design allows general structures to be defined in which chunks can be reused in multiple nonlinear segments, and the branch-point choices to be considered next can therefore depend on the path taken thus far. Ultimately, the developer should be allowed to define any arbitrary set of paths and path choices through the media, including rules that take into account the user's previous path choices. To allow for this functionality, we allow the developers to define multiple branch-point rules, including multiple rules that have the same lead-in segment (going into a branch point).

To break ties when there are multiple branch-point rules that match a particular user's current playback point, we employ a longest-path matching policy that always picks the rule that satisfies the properties of the client (part (i) of the rule) and for which the branch-point path (part (ii) of the 


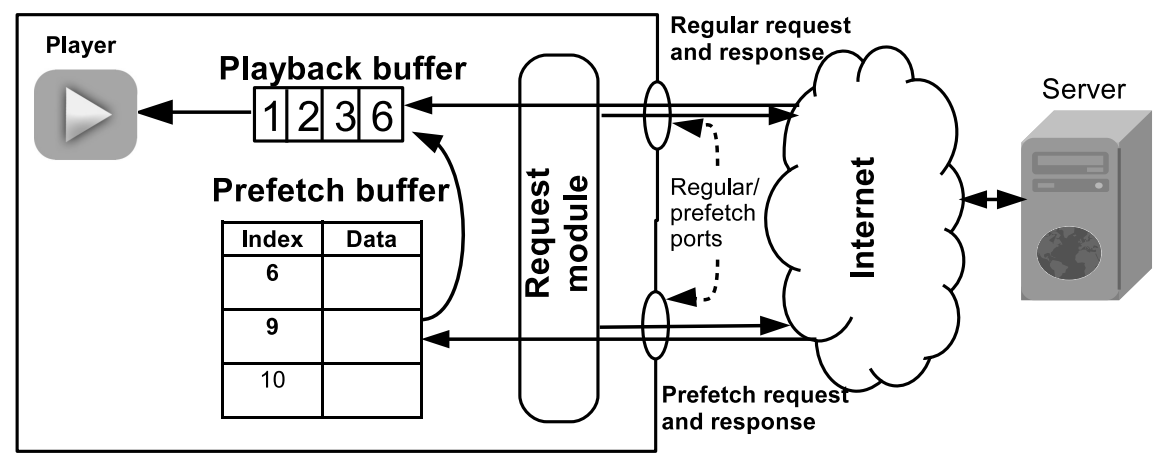

Figure 5.2: High-level design and buffer management.

rule) most closely match the path taken thus far by the client. It should be noted that this rule together with the definition of the metafile provides an easy generalization of any graph-based interactive branched structure.

\subsubsection{Prefetching and rate adaptation}

To allow seamless playback we prefetch content for each candidate path for upcoming branch points. For this purpose, we maintain a prefetch buffer, which works in tandem with the playback buffer to provide uninterrupted playback experience. Figure 5.2 illustrates the high-level design. To allow easy access to prefetched chunks associated with different paths, the prefetch buffer is implemented as a dictionary with the chunk index as the key.

A request module manages the chunk downloads. It is designed to differentiate between regular chunks, chunks at branch points, and chunks at landing segments (i.e., the candidate segments of a branch point). Based on the class that a chunk belong to, it is directed either directly to the playback buffer or is stored in the prefetch buffer. chunks belonging to the default path are handled using the default player routines, and are immediately forwarded to the playback buffer, from which they are played in the order they are inserted into the buffer. Prefetched chunks, belonging to alternative candidate segments are typically prefetched and stored in the dictionary, from which they are moved into the playback buffer only at a time when the user selects such a non-default path. At the time of such selections, our modified player replaces the pre-loaded chunks for the default path with the prefetched chunks corresponding to the selected path.

Figure 5.4 illustrates the prefetching and buffer management process for a client that is playing an interactive branched video shown in Figure 5.3 and is selecting the path along the red (dashed) arrows; in no case the default path. The request and download completion times are shown for each chunk. In this example scenario, we have used a policy in which the player requests the landing chunks of each path choice three chunks ahead 


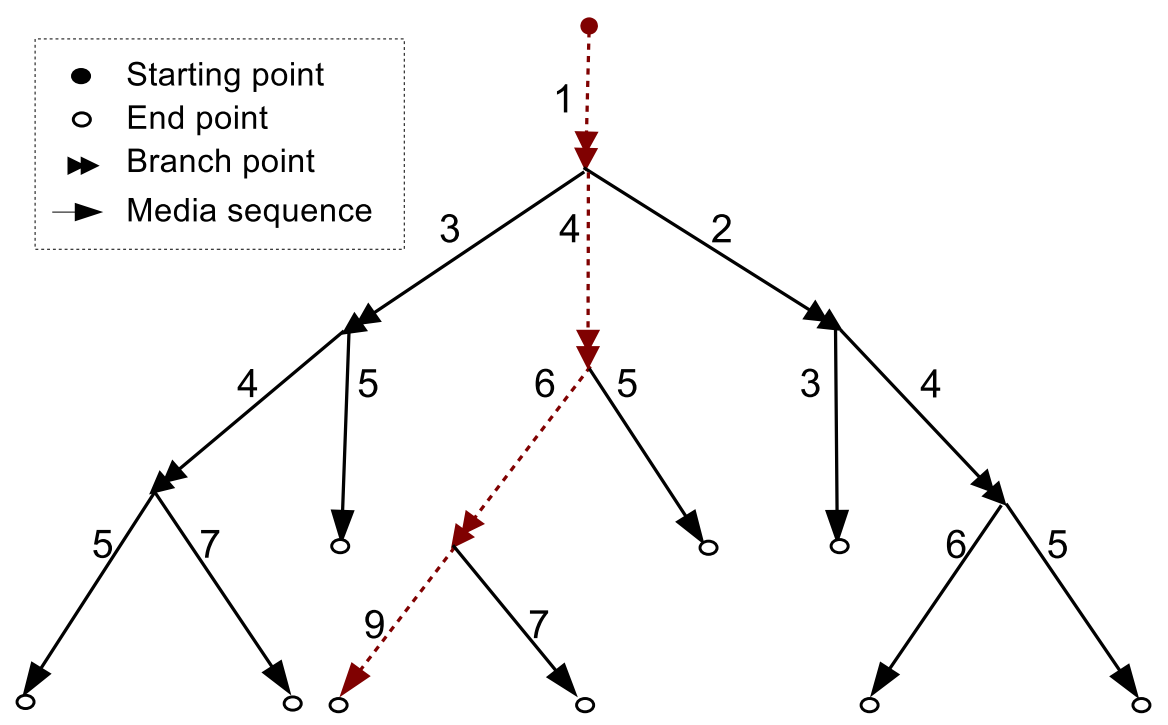

Figure 5.3: Example structure of an interactive branched video.

of reaching the next branch point.

Our request module adapts the requested video quality of both the regular requests and the prefetch requests. As with other adaptive players, we keep track of the average download rates observed over previous chunks, estimating the available bandwidth, and selecting chunks qualities based on this information. While our framework allows a variety of policies, we note that a good policy should ensure that all chunks are downloaded by the time of their (potential) playback deadlines, and yet ensuring that the playback quality is of highest possible quality. For further improvements, policies can carefully weigh the priorities of different path choices; e.g., as defined by the likelihood a path is selected.

\subsection{Implementation description}

We built an implementation of our solution using the Open Source Media Framework (OSMF) version 2.0, and its accompanying Strobe Media Playback (SMP) player.

\subsubsection{Implementation overview}

At a high level, we have added functionalities required to track, monitor, and play interactive branched videos. This includes (i) changes to ensure that we can download and read metafiles, (ii) the introduction of a request module that is responsible for prefetch requests, and (iii) the addition of 


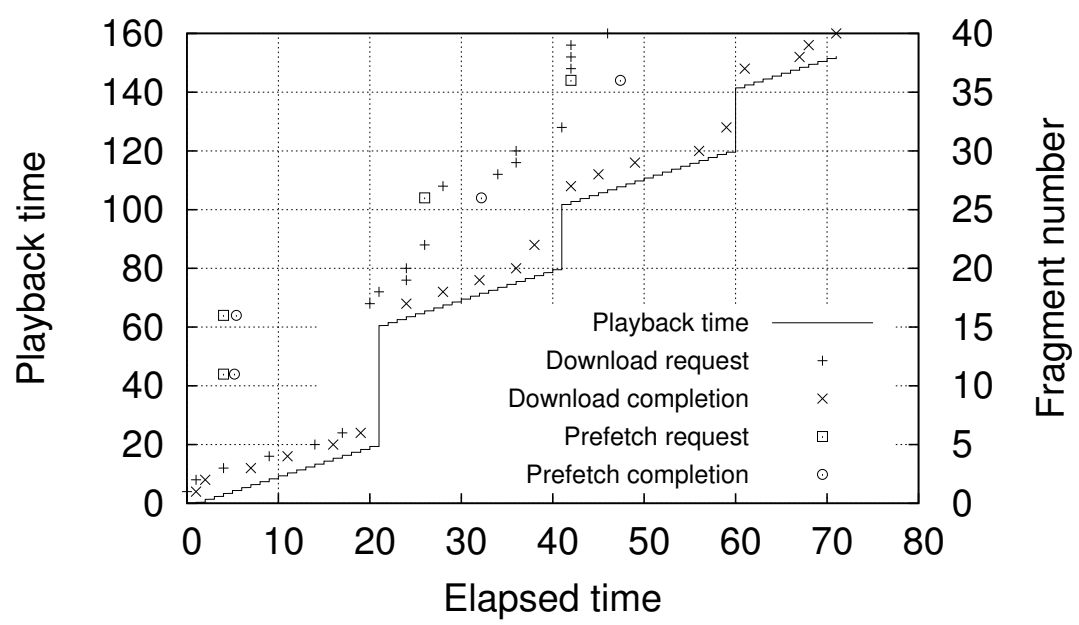

Figure 5.4: Timestamps when an example client request and download different segments.

a buffer manager module that is responsible for seamless playback when jumping between chunks in different parts of the original media object.

A new downloadManager class is responsible for downloading the branched video metafile and the manifest file of the original media, as well as controlling the request module. The request module incorporates both the HttpStreamDownloader class, which is responsible for the sequential inorder requests, as defined by the default path, and the prefetch requests. The buffer manager module works in tandem with the request module, and is responsible for controlling the chunks moved in and out of the playback buffer.

The download manager is also responsible for tracking the playback path, managing the set of branch points, and determining the next branch point to be considered. The download manager also integrates user-triggered event actions. We use a routine in the user interface (the SMP player) as a trigger for these routines. While the user in theory should have the freedom to change the path selection up until the time of the branch point, we use a $50 \mathrm{~ms}$ threshold before the end of the playback of the last chunk before the branch point to trigger these events.

Currently we have two versions of the SMP player. The first, more general, implementation allows for up to ten different path choices at each branch point, and path choices are entered using the keyboard. The last pressed key at the time the branch-point event is reached determines the selection. The second implementation assumes a binary tree, and use a button with a left-turn or right-turn arrow, which when pressed switches the turn of the arrow. Both implementations help the user to visualize the 
selected path choice, and allow changes until the branch-point is almost reached. Future work includes more user-friendly designs.

\subsubsection{Buffer management}

The interaction between our request module and buffer manager module are of critical importance in providing seamless interruption free playback. The request module feeds the playback buffer with sequential in-order chunks and the prefetch buffer with the landing chunks of the different alternative paths for the next branch point. We use a prefetch policy in which we prefetch chunks when the player is $n$ chunks before the branch point and the HttpStreamDownload class is downloading chunks along the default path directly into the playback buffer.

The playback buffer behaves like a first-in-first-out (FIFO) queue, in which the chunks downloaded are read as a byte/datastream by the SMP player in the same order they were inserted. No consideration for chunks or FLV tags are present at this time which otherwise could be used to separate different parts of the media. Unfortunately, this FIFO property limits the possibility of inserting/replacing nonlinear events, as every outof-order chunk would be queued behind already inserted in-order chunks. To allow seamless and non-interruptive playback without the user having to watch any irrelevant chunks (corresponding to non-wanted chunks along the default path, for example) which have already filled the buffer, we must therefore modify the buffer content. This functionality is implemented in our buffer management module.

At the time a non-default path is selected, the buffer management module use the HTTPNetStream class to invoke a seek event. When triggered, the seek routine immediately clears the playback buffer, and reinserts data from the point at which the user wants to seek to. In our case, we seek to the chunk just before the landing point. This would immediately cause the player to request the desired landing chunk. As we already have this landing chunk in our prefetch buffer, we can intercept this request and modify the request to the following chunk along that path. The reason for the manipulation of requests, is to preserve some of the player's internal states and to make the player consume a chunk that was not downloaded through its normal download port. As soon as this request is placed and when the player expects some bytes, we can push the data held in the prefetch buffer into the playback buffer, which immediately can be played out. In the meantime the next chunk is downloaded and is typically downloaded by the time the landing chunk is played. All requests following this can be handled in the regular manner, without any manipulation of the player state.

By prefetching data in advance and pushing it into the playback buffer exactly when it is needed, we are able to provide uninterrupted playback even in the case of nonlinear playback events. To the user, this appears as a seamless jump from one playback point to another. Of course, there are 
some minor delays associated with emptying the playback buffer, updating the player states, and inserting the already prefetched $\operatorname{chunk}(\mathrm{s})$ into the player. However, during transition points between media segments these delays are minimal and are not noticeable by the user.

\subsubsection{Rate-adaptive prefetching}

Rate adaptation is at the core of any HAS-based system. Our request module utilizes the bandwidth estimations provided by the underlying OSMF player when selecting qualities, but also takes into account that both the regular requests, downloading chunks along the default path, and the prefetch requests must share the same bandwidth.

In this chapter we present preliminary validation results for one basic example policy. With our adaptive policy, the request quality of the regular requests are adjusted based on the bandwidth that the prefetch requests will require. At a time when parallel downloads will be done, the qualities are selected such that all the paths are given at least the minimum quality, the quality of the default path is maximized, and all assignments are done under the constraint that their combined estimated bandwidth should not exceed the overall estimated available bandwidth. For validation purposes we also include results for a policy that does not perform any prefetching, and hence does not require any additional buffer management. Future work includes in-depth investigation of a range of policy alternatives.

\subsection{Proof-of-concept validation}

\subsubsection{Experimental setup}

All experiments were done in a testbed, in which we connect a client machine and a server machine over a LAN. Running dummynet at the client machine, we can manipulate the perceived bandwidth conditions, end-to-end delay, and packet losses. Our modified player is embedded in a Web page and runs on the client PC. The server runs Adobe Media Server version 5.0, and hosts a video of the animation movie Big Buck Bunny, encoded at $1300 \mathrm{~Kb} / \mathrm{s}, 850 \mathrm{~Kb} / \mathrm{s}, 500 \mathrm{~Kb} / \mathrm{s}$ and $250 \mathrm{~Kb} / \mathrm{s}$. We have also instrumented the source codes to write internal player states and information into a log file, which we post process for further analysis.

\subsubsection{Player validation}

To validate the functionality of our interactive branched player, we performed a number of validation experiments. Due to limited space we can only show a small subset.

Table 5.1 summarizes the stall-time statistics for validation experiments in which we used a fixed bottleneck bandwidths of $2 \mathrm{Mb} / \mathrm{s}$ and $3 \mathrm{Mb} / \mathrm{s}$, and 


\begin{tabular}{|l|l|c|c|}
\hline Scenario & Policy & $\begin{array}{c}\text { Late data } \\
\text { (stall events) }\end{array}$ & $\begin{array}{c}\text { Branch time } \\
(\mathrm{s})\end{array}$ \\
\hline \multirow{2}{*}{$3 \mathrm{Mb} / \mathrm{s}$} & No prefetching & $100 \%$ & 3.458 \\
& Adaptive prefetch & $0 \%$ & 0.447 \\
\hline \multirow{2}{*}{$2 \mathrm{Mb} / \mathrm{s}$} & No prefetching & $100 \%$ & 5.211 \\
& Adaptive prefetch & $0 \%$ & 0.547 \\
\hline
\end{tabular}

Table 5.1: Stall events and branch times.



Figure 5.5: Playback quality.

the client used the test path in Figure 5.3, with each segment consisting of five chunks. Figure 5.5 presents the video quality statistics for these experiments. Average values are presented over five experiments.

We can see that our adaptive policy is able to completely eliminate any playback interruptions due to the data not being delivered to the client in time. At this time, the only waiting times the player will endure is the time that it takes to clear the buffer and reload the prefetched content. We see that this time is typically around $0.5 \mathrm{~s}$, out of which $0.23-0.25 \mathrm{~s}$ is to load the content and the rest is to update various internal states in the player. We have found that this time is not noticeable to the human eye, as long as we shift scene.

Our initial results are encouraging. However, many interesting performance tradeoffs remains to be considered, including tradeoffs between video quality and potential playback interruptions. Future work includes the development and performance evaluation of more advanced buffer management and rate adaptation policies. 


\subsection{Conclusion}

This chapter presented a novel design that leverages the chunk-based nature and differentiated quality levels of HAS to define and achieve seamless streaming of interactive branched video. Personalization of the content can be achieved with the use of a customized metafile. Our overall solution is simple and provides the creative home user with the power to create inventive interactive branched, and nonlinear videos and to explore new media forms. While preliminary validation results are presented, future work includes more careful exploration and performance evaluation of more advanced buffer management, prefetching, and rate adaptive policies. 


\section{Chapter 6}

\section{Prefetching strategies for interactive branched video streaming over HAS}

\subsection{Introduction}

On-demand video streaming has gained tremendous popularity over the last few years and today contributes the majority of Internet traffic [128]. In contrast to the original UDP-based services, most of today's on-demand video streaming services are using HTTP over TCP. The use of HTTPbased streaming simplifies caching and facilitates simple traversal of NATs and firewalls. With the introduction of HTTP-based adaptive streaming (HAS) protocols, the player can now also easily adapt the streaming quality based on the user's current bandwidth conditions [25, 120].

In addition, we have also witnessed an increased level of personalization on the Web. As companies and users are becoming increasingly used to personalized services, we expect increasing personalization of video. For example, a viewer may find a movie too sad, too violent, or too scary for their liking. What if the content provider could customize the video playback sequence based on the taste of each client?

Interactive branched videos that allow users to traverse different plot sequences, depending on their interactions with the video (e.g., by pushing a button on a keyboard, TV remote, or with the help of a mouse click) have been proposed in the research literature $[6,103,106]$, and explored through clever (but non-trivial) use of navigation captions in YouTube videos. Recently, Interlude ${ }^{1}$ even launched a Web-based service that offers content

\footnotetext{
${ }^{1}$ Interlude, http://www.interlude.fm, Mar. 2014.
} 
creators an easy way to create interactive videos hosted by the company.

While these types of media provide many advantages and opportunities, they also present new challenges. For example, with the user playback experience (at least of linear video) being dominated by the number and durations of any potential playback interruptions [15], branched video presents a unique challenge as the player may not know which potential path a client will take until reaching that playback point. Therefore, the video must be prefetched along multiple paths to ensure that the playback is seamless, without playback interruptions even when the users defer their choices to the last possible moment.

We argue that HTTP-based Adaptive Streaming (HAS) provides an excellent framework for implementing interactive branched streaming (Section 6.2). Using a simple media description and path tracking mechanism (i) provides the creator with arbitrary flexibility when designing personalized content, (ii) does not add any additional overhead on the content servers storing the media files compared to users watching regular linear non-interactive media, and (iii) the use of HAS ensure that the solution is TCP friendly, easily traverses firewalls/NATs, and can leverage all the benefits of content replication and proxy caches.

In this chapter, we describe a design and full implementation of such a HAS-based interactive branched streaming system. Using a relatively simple model and this implementation, we explore the key question of what policy a branched player should use when deciding what pieces to download when. Such a policy is analogous to the rate adaptation policy in HAS, but is made greatly more complex by uncertainty regarding future user path selections. The contributions of this chapter are as follows.

We first develop a simple analytic model that allows us to define the basic prefetch problem as an optimization problem in which we maximize the expected playback quality, while avoiding playback interruptions. Within this framework we show that under several realistic circumstances it is optimal to download back-to-back video chunks in a round-robin manner, and that there exists a natural tradeoff between playback quality and the use of additional parallel TCP connections to build up buffer workahead and ensure seamless playback.

Second, based on these findings, we design optimized policies that determine (i) when different parts of the video should be downloaded, (ii) what quality level each such part should be encoded with, and (iii) how to manage playback buffers and TCP connections such as to ensure smooth playback experience without excessive workahead. By extending the existing buffer management policies used for HAS our policies carefully balance the buffer workahead needed to ensure seamless playback against potential wasted bandwidth associated with prefetching data along non-used paths or for an early terminated session, for example.

Finally, we present the design, implementation (Section 6.4) and experimental evaluation (Section 6.5) of our framework. In addition to providing 
concrete evidence that the optimized policies perform well under a wide range of scenarios, these experiments provide insights into the importance of careful adaptive policies and workahead. For example, it is shown that the use of parallel connections can be particularly valuable when building workahead in environments with much competing traffic, and our optimized prefetching policies together with a capped workahead policy is shown to provide a good tradeoff between ensuring smooth playback experience and avoiding excessive workahead.

The remainder of this chapter is organized as follows. Section 6.2 presents our general HAS-based interactive branched framework. Section 6.3 defines our system model, problem formulation, and optimized prefetching policies. Section 6.4 presents our system design implementation, before Section 6.5 presents our experimental evaluation. Section 6.6 concludes the chapter.

\subsection{Personalized content delivery}

\subsubsection{Chunks, segments, and branch points}

HAS players typically either use byte-range requests or split the file into individually addressable chunks. While the general design in this chapter is applicable to both types, we will present a chunk-based solution. With chunked video, the video is split into smaller chunks, and each chunk is addressable by a unique URL. A manifest file providing URLs for individual chunks is used to bootstrap the player. Each chunk is typically 2-5 seconds long, and chunk boundaries coincide for the different encodings, allowing the client to switch to any available encoding at chunk boundaries.

The interactive branched video that we present here generalizes traditional linear HAS video in that it allows (i) the video designer to define arbitrary playback sequences through the underlying linear video, and (ii) the users to select among multiple alternative playback sequences. In order to allow for such customization and interactivity, the designer can define both linear and nonlinear segments, that are linked together using branch points, at which the user can chose among multiple path options [6].

Figure 6.1 illustrates our terminology. Here, as with regular HAS video, a chunk contains a small fixed duration of the video and is defined within the video manifest file. Each segment (linear or nonlinear) consists of a sequence of chunks, that are played in the specified order without the need for any user interaction. Branch points are used to give users the option of alternative video playback paths. While playing the segment leading up to an applicable branch point, the user is given the option of which of several possible next segments the player should play next. 
Chunks

\begin{tabular}{|l|l|l|l|l||l|l|l|l|}
\hline 1 & 2 & 3 & 4 & 5 & 6 & 7 & 8 & 9 \\
\hline
\end{tabular}

\section{Linear \\ segments}

Non-linear

segments

Branched video
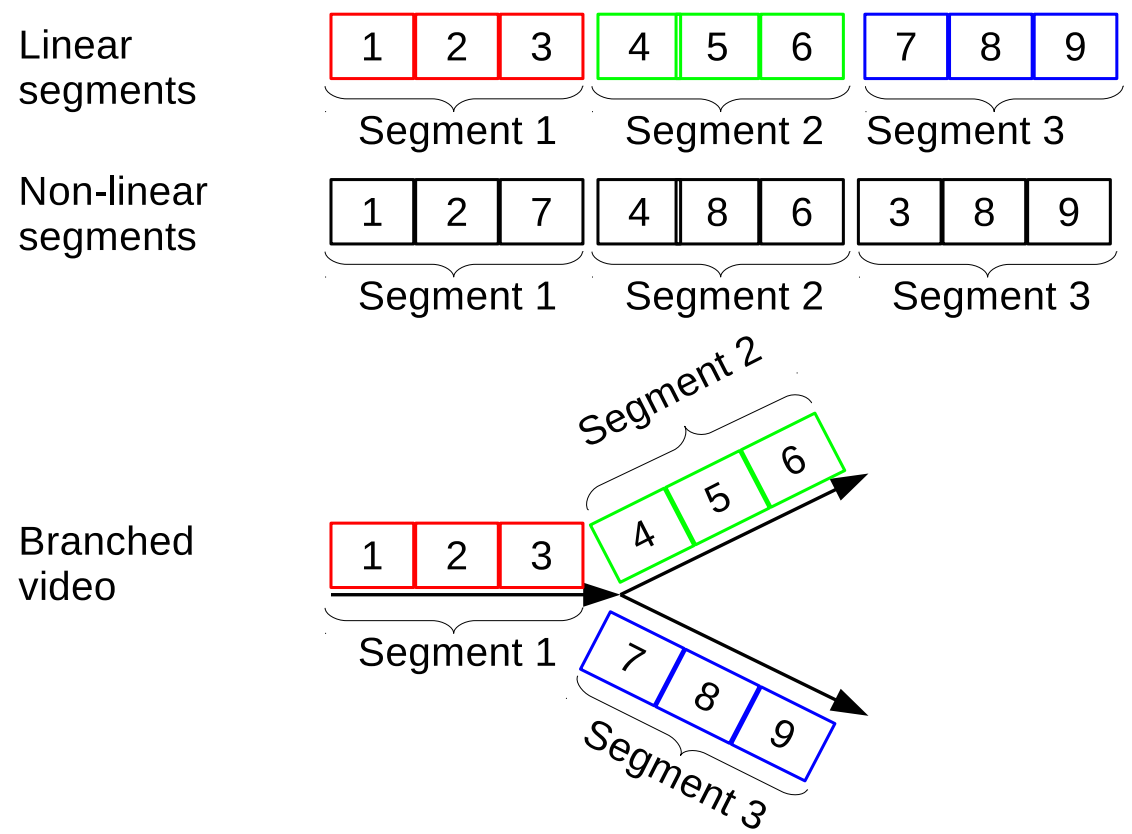

Figure 6.1: Illustration of terminology.

\subsubsection{Interactive branched, nonlinear media structure}

Our client-driven design does not add any client tracking or additional processing overhead for HAS servers. The designer simply specifies segments and branch point rules in a text-based metafile, separate from the manifest file that comes with the underlying HAS media. This personalized metafile can be individually downloaded from a separate source (e.g., from the content designer) and the player is responsible for determining which branch points to consider and chunks to download.

Segments can be specified as any arbitrary sequence of chunks and the applicable branch points can be defined using very rich branch point rules that specify [6]: (i) the playback path that the client must have taken for the branch point rule to be applied, (ii) the path options associated with the branch point, (iii) the weights associated with each path choice, and (iv) the message to be displayed to the user whenever that branch point is in play. When playing the video, our player keeps track of the playback sequence of the user and compares it with each of the playback path that the client must have taken for the branch point rule to be applied. Among 
all matching branch point rules, the player picks the one with the longest match. In the remainder of this chapter we will focus on how the player performs prefetching, given the outcome from applying this rule.

Consider an interactive branched media file that consists of $E=|\mathcal{E}|$ nonlinear segments and $B=|\mathcal{B}|$ applicable branch points. Here, each nonlinear segments $e \in \mathcal{E}$ is of playback length $L_{e}$ and consists of $n_{e}$ chunks each with playback duration $l_{e, i}$, where $1 \leq i \leq n_{e}$. In later sections we will also leverage that each chunk can be downloaded in one of $Q=|\mathcal{Q}|$ qualities, where $\mathcal{Q}$ is the set of qualities, each corresponding to a particular video bitrate. We use $q_{e, i}$ to denote the quality (bitrate) of chunk $i$ of segment $e$, and hence the size of this chunk is $q_{e, i} l_{e, i}$.

Each applicable branch point $b \in \mathcal{B}$ has $\mathcal{E}^{b}$ branch options that can be selected, each given a weight $w_{e}^{b}$. In the following, we will assume that all weights are normalized, such that $\sum_{e \in \mathcal{E}^{b}} w_{e}^{b}=1$, and the weights reflect their relative priorities (e.g., the probability that a path is selected). Table 6.1 summarizes our notation.

\subsection{Player management}

\subsubsection{Problem description}

We design optimized prefetching policies and buffer management schemes that (i) provide uninterrupted playback with seamless switches from one segment to another, and (ii) given such uninterrupted playback, maximize the playback quality. Our HAS-based solution takes advantage of parallel TCP connections to build up a workahead buffer to keep unnecessary stalls at a minimum, but must also make careful tradeoffs among workahead, playback quality, and the potentially wasted bandwidth associated with downloading chunks the client later does not use.

As an illustration of these design choices, consider first Figure 6.1. Assume that the player is currently playing segment 1 . To ensure seamless playback when reaching the branch point, the first chunk of each path choice (i.e., chunks 4 and 7) must have been retrieved before reaching the branch point. To make good prefetch decisions, the client must keep track of how much time there is until the branch point, and how much bandwidth is available, so as to determine when and at what quality each chunk should be downloaded.

Figure 6.2 illustrates a more complex branched structure in which the client must make multiple path decisions (bottom half of the figure), the download schedule (middle) and the playback schedule (top) for an example player implementing a basic policy. Note how all chunks along the selected (solid lines) playback paths are obtained in time of their playback deadlines (i.e., the time by which the player needs that chunk for it to be played without a stall), how up to three parallel connections were used to build up a workahead and prefetch the first chunk of each potential path choice 
Table 6.1: Notation for interactive branched nonlinear media

\begin{tabular}{|c|l|}
\hline Symbol & Definition \\
\hline \hline $\mathcal{E}$ & Set of all nonlinear segments $e$ \\
\hline$L_{e}$ & Total media playback length of segment $e$ \\
\hline$n_{e}$ & Total number of chunks in segment $e$ \\
\hline$l_{e, i}$ & $\begin{array}{l}\text { Total media playback length of chunk } i \text { on } \\
\text { segment } e\end{array}$ \\
\hline $\mathcal{Q}$ & Set of quality encodings \\
\hline$q_{e, i}$ & $\begin{array}{l}\text { The quality encoding of chunk } i \text { on segment } \\
e\end{array}$ \\
\hline $\mathcal{B}$ & Set of all branch points $b$ \\
\hline $\mathcal{E}^{b}$ & $\begin{array}{l}\text { Set of branch edges } e \text { associated with branch } \\
\text { point } b\end{array}$ \\
\hline$w_{e}^{b}$ & $\begin{array}{l}\text { Relative weight for branch-point edge } e \text { of } \\
\text { branch point } b\end{array}$ \\
\hline$\Delta^{u}$ & Protocol threshold time used by client $u$ \\
\hline$t_{i}^{s}$ & Start of download of chunk $i$ \\
\hline$t_{i}^{c}$ & Download completion of chunk $i$ \\
\hline$t_{i}^{d}$ & Playback deadline of chunk $i$ \\
\hline$l_{\{e, b\}, i}$ & $\begin{array}{l}\text { Total media playback length of chunk } i \text { on } \\
\text { segment-branch-point pair }(e, b)\end{array}$ \\
\hline$q_{\{e, b\}, i}$ & $\begin{array}{l}\text { The quality encoding of chunk } i \text { on segment- } \\
\text { branch-point pair }(e, b)\end{array}$ \\
\hline$r_{i}$ & $\begin{array}{l}\text { Estimated per-connection download rate be- } \\
\text { tween download initiation times } t_{i}^{s} \text { and } t_{i+1}^{s}\end{array}$ \\
\hline$c_{i}$ & $\begin{array}{l}\text { Number of parallel connections between } \\
\text { download initiation times } t_{i-1}^{s} \text { and } t_{i}^{s}\end{array}$ \\
\hline$R_{i}(c)$ & $\begin{array}{l}\text { Estimated download rate at time } i \text { for client } \\
\text { using } c \text { connections }\end{array}$ \\
\hline & \\
\hline
\end{tabular}

(chunks 4, 7, and 10 at the first branch point, and then chunks 13 and 16 at the second branch point) before reaching the branch points, as well as how different qualities have been selected for the different chunks depending on how much workahead is available before each (potential) playback deadline. In this scenario we have also used a simple round-robin schedule for determining which chunk to download next.

This example illustrates the importance of careful prefetching. Important design considerations in determining a good download schedule include:

- Download ordering and playback quality. With different chunks being associated with paths of different likelihood and with different delay constraints, both the order and quality that each chunk is downloaded play an important role in ensuring seamless playback at highest possible playback quality.

- Concurrent downloads. To allow improved download speeds and 


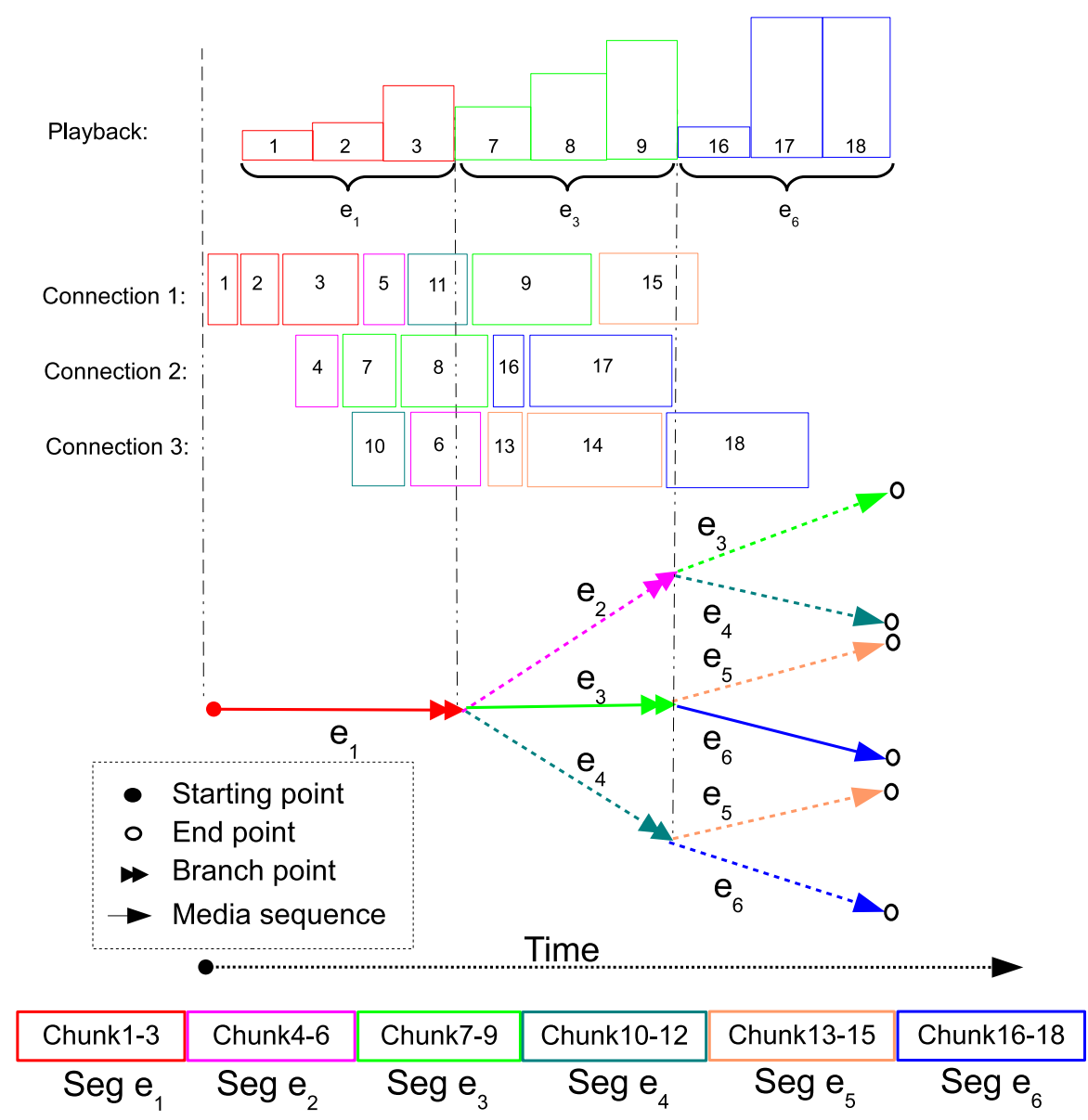

Figure 6.2: Round-robin parallel downloading

prefetching we allow multiple parallel connections. However, when doing so, we must consider the tradeoff between improving the overall download speed and meeting individual playback deadlines.

- Capped workahead. To avoid excessive workahead and wasted bandwidth usage when a user terminates a session early, most HAS players use on-off switching, and do not allow the buffer to fill up beyond a certain threshold. In the context of interactive branched video this concept must be extended to take into account the number of path options at each applicable branch point. Depending on the underlying structure, our player sets the target buffer size dynamically.

In each step, the player must determine when the client should download each chunk and how much parallelism should be utilized, such as to max- 
imize the playback quality and meet playback deadlines. Given a network constraint on how much data can be downloaded over a given time period, there is a tradeoff between quality (higher quality implies larger chunks) and the number of chunks that can be downloaded. The stall probability depends on network conditions and the prefetching policy (such as concerning prefetching of chunks from after a branch point).

\subsubsection{Objectives and constraints}

In this section we formulate the prefetching problem as a optimization problem. Without loss of generality, we consider a client downloading a single segment $e$ that has been determined to have an upcoming branch point $b$. We want to maximize the playback quality of the chunks of the current segment $e$ and the first chunk of each of the $\left|\mathcal{E}^{b}\right|$ different path choices, conditioned on each chunk, including the first chunk of each potential segment following the branch point, being downloaded prior to its (potential) playback deadline.

Objective function: Assuming that a client picks path $e^{\prime} \in \mathcal{E}^{b}$ at branch point $b$ with a probability $w_{e^{\prime}}^{b}$, we can formulate our objective function as:

$$
\operatorname{maximize} \sum_{i=1}^{n_{e}} q_{e, i} l_{e, i}+\sum_{e^{\prime} \in \mathcal{E}^{b}} w_{e^{\prime}}^{b} q_{e^{\prime}, 1} l_{e^{\prime}, 1} .
$$

Round-robin ordering: Without loss of generality, we enumerate the chunks associated with a segment $e$ and the first chunk of each of these candidate segments as follows: (i) the chunks of segment $e$ are enumerated from 1 to $n_{e}$, and (ii) the chunks belonging to the upcoming branch point $b$ are enumerated from $n_{e}+1$ to $n_{e}+\left|\mathcal{E}^{b}\right|$ with $n_{e}+1$ having the highest weight $w_{e^{\prime}}^{b}$ and $n_{e}+\left|\mathcal{E}^{b}\right|$ the lowest. With this numbering, let $q_{\{e, b\}, i}$ and $l_{\{e, b\}, i}$ be the quality and chunk length, respectively, of the $i^{\text {th }}$ chunk of the combined segment-branch-point pair $\{e, b\}$.

Ignoring delay constraints, we note that it is never better to download high-quality versions of the chunks of segment $e$ than any of the first chunks of the branch options, and it is never better to download a later numbered branch chunks (with less weight) at a higher quality than an earlier numbered chunks (with more weight). Furthermore, assuming this relative chunk numbering and non-increasing quality assignment to chunks, we note that it is optimal to initiate download of chunks in an round-robin order. To see this, note that any feasible schedule with an alternative download order (but non-increasing quality assignment to chunks) also would be feasible if the download order of any pair of out-of-order chunks where corrected.

Motivated by the above observations, in the following we only consider round-robin schedules following the above order. In fact, in the case that additional workahead is possible after downloading all the $\left|\mathcal{E}^{b}\right|$ chunks and our workahead rule (Section 6.3.4) allows for greater workahead beyond the 
branch point, we again use round-robin among the second chunk of each candidate segment.

With the above chunk order and notation, we can rewrite the objective function as:

$$
\operatorname{maximize} \sum_{i=1}^{n_{e}} q_{\{e, b\}, i} l_{\{e, b\}, i}+\sum_{i=n_{e}+1}^{n_{e}+\left|\mathcal{E}^{b}\right|} w_{e}^{b} q_{\{e, b\}, i} l_{\{e, b\}, i} .
$$

Playback deadlines: For the purpose of our timing constraints, let $t_{i}^{s}$ represent the time at which the client initiates download of chunk $i$. Similarly, let $t_{i}^{c}$ and $t_{i}^{d}$ represent the times at which the client completes download of chunk $i$ and the playback deadline of chunk $i$, respectively.

Our first timing constraint simply states that the download completion time $t_{i}^{c}$ of each chunk $\left(1 \leq i \leq n_{e}+\left|\mathcal{E}^{b}\right|\right)$ must be before the playback deadline of that chunk:

$$
t_{i}^{c} \leq t_{i}^{d}= \begin{cases}\tau+\sum_{j=1}^{i-1} l_{e, j}, & \text { if } 1 \leq i \leq n_{e} \\ \tau+\sum_{j=1}^{n_{e}} l_{e, j}, & \text { if } n_{e}<i \leq n_{e}+\left|\mathcal{E}^{b}\right| .\end{cases}
$$

Here, $\tau$ is used to indicate the time at which the first chunk of the segment is played out. If we measure time from the time $t_{1}^{s}=0$ that the download of the first segment is initiated, $\tau$ corresponds to the startup delay. For later segments, $\tau$ corresponds to the relative time at which the preceding branch point was reached, and is equal to $\tau=t_{1}^{d}=\tau^{\prime}+\sum_{j=1}^{n_{e^{\prime}} l_{e^{\prime}, j}}$, where $\tau^{\prime}$ is the corresponding value for segment $e^{\prime}$ that was traversed before the current edge $e$.

Startup delays: For simplicity, we use the minimum possible startup delay if all segments were downloaded sequentially at some minimum desired quality $q^{*}$. In this case, the startup delay $\tau$ can be calculated as the maximum of $\max _{1 \leq i \leq n_{e}}\left[\sum_{j=1}^{i} \frac{q^{*} l_{e, j}}{R(1)}-\sum_{j=1}^{i-1} l_{e, j}\right]$ and $\sum_{j=1}^{n_{e}+\left|\mathcal{E}^{b}\right|} \frac{q^{*} l_{\{e, b\}, j}}{R(1)}-$ $\sum_{j=1}^{n_{e}} l_{e, j}$, where $R(1)$ is the download rate when using a single TCP connection. Here, the first expression finds the tightest constraint for each of the segments of the edge $e$ itself (typically suggesting a startup delay equal to the download completion of the first segment $\tau=t_{1}^{c}$ ) and the second expression corresponds to the constraint of obtaining the first chunk of each branch choice in time of playback. The same method is again used anytime that we have a playback interruption and $\tau$ must be re-calculated.

Bandwidth constraints: Our final set of constraints relate the bandwidth available to each of the client's connections, the amount of a chunk it can download at each point in time, and how long it will take to download the chunk. For these constraints, we will restrict our policies to those that only initiate new chunk downloads and open new TCP connections at the time of a download completion. Such policies simplify connection management.

With a measured combined download rate of $R\left(c_{i}\right)$ between the initiation of the $i^{\text {th }}$ and $(i+1)^{\text {th }}$ chunk downloads, the average download rate 
per connection is equal to $r_{i}=R\left(c_{i}\right) / c_{i}$, where $c_{i}$ is the number of active parallel connections during this time period. Now, assuming that these perconnection rates $r_{i}$ are known for each time instance, we can formulate the following conservation equation:

$$
\sum_{j=i}^{i+k} r_{j}\left(t_{j+1}^{s}-t_{j}^{s}\right)=q_{e, i} l_{e, i}
$$

where we have assumed that $k$ new chunk downloads are initiated before the $i^{\text {th }}$ chunk is fully downloaded; i.e., $t_{i}^{c}=t_{i+k}^{s}$. Here, the left-hand side gives the total amount of data downloaded on a single connection during the download time of a particular chunk, recognizing that the download rate on that connection will change if the number of active parallel connections changes. The right-hand side gives the size of that chunk.

Rate constraints: In practice, neither $r_{j}$ or any of the future download instances are known at the time of a download completion, when the player must determine (i) how many parallel connections to use next until the next completion, and (ii) at what quality any new chunks downloads should have. At one extreme the client has no competition. In this case $R(c)$ is independent of $c$ and $r_{i+1}=\frac{c_{i+1}}{c_{i}} r_{i}$. At the other extreme, the client has very many competing flows, $R(c)$ is approximately proportional to $c$ and $r_{i+1}=r_{i}$ regardless of the value of $c_{i}$ and $c_{i+1}$.

To help ensure that delay constraints are satisfied even under uncertainty, our policies use the conservative assumption that there will be no additional bandwidth gains from using additional TCP connections, but prioritize parallelism when possible without violating these delay constraints.

\subsubsection{Prefetching policies}

The player initially opens one connection to the server, and subsequently opens additional connections at download completion time instants, depending on the prefetch policy. Idle connections are reused, and, as shown in Figure 6.2, HTTP GETs for different branches can share a single connection.

To determine the number of new chunks to request next, and the quality at which these chunks should be downloaded, we have found that the above constraint problem is best solved one segment at a time. At the completion of a chunk download, we pick the quality and number of parallel new downloads that maximizes the expected weighted playback quality, as defined by the objective function (6.2), and that satisfies all the above constraints.

We consider two policies that both restrict the number of candidate schedules to consider, but differ in their complexity and how aggressive they are. The first policy, called Optimized non-increasing quality, considers all possible candidate schedules that (i) do not exceed the maximum number of parallel connections allowed $C$, (ii) do not count on opening additional 
Table 6.2: Summary of prefetching policies.

\begin{tabular}{|c|c|c|l|}
\hline Policy & Connections & Schedules considered & Objective \\
\hline $\begin{array}{c}\text { Optimized non-increasing } \\
\text { quality }\end{array}$ & $1 \leq c_{i} \leq C^{\max }$ & $\begin{array}{c}M^{Q}, \text { where } \\
M=n_{e}+\left|\mathcal{E}^{b}\right|-m\end{array}$ & Equation $(6.2)$ \\
\hline $\begin{array}{c}\text { Optimized maintainable } \\
\text { quality }\end{array}$ & $1 \leq c_{i} \leq C^{\max }$ & $\left(\begin{array}{c}M+Q-1 \\
Q-1\end{array}\right)$ & Equation $(6.2)$ \\
\hline Single connection & 1 & $\left(\begin{array}{c}M+Q-1 \\
Q-1\end{array}\right)$ & Equation $(6.2)$ \\
\hline Greedy bandwidth & $1 \leq c_{i} \leq C^{\max }$ & $\left(\begin{array}{c}M+Q-1 \\
Q-1\end{array}\right)$ & $\sum_{i=j}^{j+m} q_{e, i} l_{e, i}$ \\
\hline
\end{tabular}

connections in future steps, and (iii) in which the qualities of consecutive chunks are non-increasing. This policy reduces the number of possible schedules from $M^{Q}$, where $M=n_{e}+\left|\mathcal{E}^{b}\right|-m$ is the number of remaining chunks, $m$ is the number of already requested chunks, and $Q$ is the number of possible quality levels for the segment-branch pair $(e, b)$, down to $\left(\begin{array}{c}M+Q-1 \\ Q-1\end{array}\right)$.

As condition (iii) still can allow for a large number of candidate schedules, we also consider a more conservative policy that requires the quality of the requested chunks to be sustainable for the remaining chunks. This policy, called Optimized maintainable quality, requires only $Q$ candidate schedules to be considered per number of parallel connections. Both policies pick the highest feasible quality for the next chunk, and open up additional parallel connections only if this quality can still be achieved after doing so.

To explore the tradeoff between aggressively picking high quality segments and prioritizing opening up additional parallel connections, we also consider two additional prefetching policies. In the first policy, called Single connection we use the same quality assignment candidates as with the Optimized maintainable quality policy, but only allow a single connection to be open at each point in time. Finally, we consider a much more bandwidth aggressive policy, called Greedy bandwidth, in which the quality and number of parallel new downloads maximize the number of requested bytes $\sum_{i=j}^{j+m} q_{e, i} l_{e, i}$, when chunks $j$ through $j+m$ are requested at this time instance. To allow easier head-to-head comparison we again use the three candidate schedule constraints used with the Optimized maintainable quality policy. Table 6.2 summarizes our policies.

\subsubsection{Capped workahead}

Most HAS players perform on-off throttling. A common strategy is to stop making chunk requests whenever the buffer occupancy exceeds a certain threshold $T_{\max }$, until the buffer occupancy drops below another threshold $T_{\min }[24,25]$.

We generalize this idea to the context of interactive branched video. Consider a player that is currently playing a segment $e \in \mathcal{E}$ and is approaching branch point $b$ with $\mathcal{E}^{b}$ alternative branches. We define a minimum buffer threshold $T_{\min }=T_{\text {single }} \cdot\left|\mathcal{E}^{b}\right|$, where $T_{\text {single }}$ is the amount of data that 
should be buffered for each potential branch segment to be comfortable that stalls do not occur, but such as to avoid excessive buffering along that path, in the case that path was not selected. Using the minimum buffer threshold, we then simply set the maximum buffer threshold $T_{\max }=\left(T_{\min }+\Delta\right)$, where $\Delta$ is additional buffer margin.

\subsection{Implementation description}

\subsubsection{Software overview}

Our client is implemented using the Open Source Media Framework (OSMF v2.0) libraries and the front-end player is built from Strobe Media Playback (SMP). While most modifications are focused on the classes under the httpstreaming directory, responsible for HAS, some modifications to the files under the StrobeMediaPlayback directory were needed to implement user interaction ${ }^{2}$.

\subsubsection{High-level system overview}

After having downloaded the HAS manifest file (that describes the HAS media file and the different encodings at which the chunks are available) and the interactive branched metafile (that describes the branched structure), the client bootstraps the player using the information from the two files.

Our modified HAS player can open parallel TCP connections, request and download multiple chunks in parallel. The chunks along the current playback segment are delivered in-order to the playback buffer, whereas the prefetched chunks associated with the upcoming branch segments are downloaded into the browser cache, from which they can be very quickly retrieved when the client makes a path decision. This design has been tested and compared against in-player memory solutions such as those presented by Krishnamoorthi et al. [6], showing significant reductions in in-player memory requirements and most importantly in the time that it takes to load a new path. This is important when masking the load times from cache to player, so as to ensure that users do not observe any playback interruptions.

Finally, to minimize fetch times from the cache, our current implementation uses the browser RAM cache (of our Mozilla Firefox version 25.0.1 browser), and requests from the player are checked against the index of cache content before placing a request to the server. While we have found benefits to using a RAM cache, relative to the use of a disk cache, these differences are negligible compared to the overall benefits of prefetching to cache in general.

\footnotetext{
${ }^{2}$ Our data files and source code for our software framework are available at http: //www.ida.liu.se/ nikca/papers/mm14.html
} 


\subsubsection{Interactive playback}

Once playback is close to a branch point, a message is displayed to the user requesting selection of the desired path option. The player reads the user input and this information is communicated to the class that handles branch-point transitions. The player also maintains a record of chunks and segments it has played previously. This information is used whenever the player has to use the longest matching rule (Section 6.2.2).

Once playback reaches a branch point, a branch-point event is triggered. Using the time associated with the start of the selected branch, the player calculates the chunk that should be played first for this branch. Using this chunk number, our modified player checks if any such chunk has been requested, and if so, determines its URL. Having determined the cached URL, the player can now quickly retrieve the content and move it into the playback buffer.

One of the most influential parameters in determining the duration of any playback interruptions at a branch point is the BufferTime value. This amount represents the minimum amount of data (in seconds) the player needs to have in the playback buffer before resuming playback after a stall or at a branch-point event. Whenever our download manager triggers a branch-point event, we therefore set the BufferTime value to a small value so that it can resume playback right away. As soon as the seek is complete the BufferTime value is reset to the player's default value. When playback is resumed after a branch point event, additional requests are placed so as to fetch all the prefetched content for the new segment from the cache, before the request module resumes its normal operation and continues prefetching of the remaining chunks along the new segment and any chunks associated with the next upcoming branch point.

\subsubsection{Parallel connections}

An important aspect of allowing seamless playback is effective workahead prefetching. Researchers have found that HAS players often experience large degradation in performance when exposed to competing flows [23, 24, 129]. Based on initial experiments and related literature (e.g., [130]) we conjecture that multiple parallel TCP connections will help ensure stable throughput in such scenarios.

As described in Section 6.3 we schedule new chunk downloads and open new TCP connections based on our workahead prefetching policies, which take into account playback constraints, current download rate estimates, and the priority order of the chunks. Our policies are conservative, in that when calculating expected download completion times it is assumed that an additional TCP connection will not increase the total achieved download rate (and so would correspondingly reduce the rate achieved by each of the existing connections). At the end of each chunk download, new parallel connections are only initiated if they are not expected to violate the deadline 
constraints of both chunks currently being downloaded and chunks to be downloaded.

\subsubsection{Rate estimation}

Rate estimation is a critical component of any HAS player. Typically, rate estimates of the available bandwidth are generated based on the download rates observed during previous downloads. To avoid placing too much weight on the most recent chunk download, most HAS players maintain a rolling window and/or calculate a weighted average of the estimated download rate, based on past download times.

Similarly, with our interactive branched player, rate estimates are calculated by measuring the time that it takes to download each chunk. However, we must also account for the fact that a chunk may have been downloaded in parallel with other chunks. For this reason, each new estimate is scaled up accordingly, using the conservative approximation that the number of such parallel connections was constant and equal to the minimum number of parallel connections during the download of the chunk. As with the default player, these estimates are then used to update a global estimate, calculated using an exponentially weighted moving average (EWMA) with weight 0.4.

\subsection{Experimental results}

\subsubsection{Experimental setup}

To validate our general system design and evaluate the performance of different prefetching policies, we use a basic experimental testbed, in which a client and the server are connected over a high-speed LAN. To emulate a wide range of network conditions the client machine runs dummynet [123], allowing us to control the available bandwidth, the RTT, and the packet loss rate observed between the two machines.

The server runs a Flash Media Server (FMS) version 4.5, and hosts a HAS video chunked into $4 \mathrm{~s}$ chunks and encoded into four different playback qualities, encoded at $250 \mathrm{~Kb} / \mathrm{s}, 500 \mathrm{~Kb} / \mathrm{s}, 850 \mathrm{~Kb} / \mathrm{s}$, and $1300 \mathrm{~Kb} / \mathrm{s}$, respectively. These encodings were generated from the Big Buck Bunny video using Adobe's encoding and HAS packaging suite. For the purpose of discussion we sometimes refer to these qualities as quality $0,1,2$ and 3 , respectively. Branch points and segments were generated arbitrarily from the base video.

The player is instrumented to continually write its internal state and actions to a $\log$ file, which we later process to evaluate the performance experienced by the client. Of particular interest is the buffer occupancy, the frequency and duration of stall events, as well as the general playback rate and quality experienced by the client.

The performance of our system design, is evaluated under each of the four prefetching policies Optimized non-increasing quality, Optimized main- 
tainable quality, Single connection, and Greedy bandwidth, all defined in Section 6.3.3, as well as a Naïve player that is capable of handling interactive branched video, but that does not perform prefetching along the different alternative paths, but simply downloads chunks only along the current default path. Naturally, this policy stalls at each branch point that the default path is not selected and simply serves as a baseline for comparison.

By comparing the two optimized policies, Optimized non-increasing quality and Optimized maintainable quality, we can evaluate the value of being conservative, as the second policy should be considerably more conservative in its quality selection for the next segment. By comparing the performance of this policy with the Single connection policy, we can assess the value of using parallel connections. Finally, Greedy bandwidth helps further capture the tradeoffs associated with aggressively opening up parallel connections.

In the following subsections we present experiments in which we vary one parameter at a time, starting from a default scenario, such as to give initial insights into the impact that factors such as the available bandwidth, end-to-end RTTs, number of chunks per segment, number of branches, and amount of competing traffic have on the playback performance. Results are presented both for the first branch point and for later branch points. For Sections 5.2-5.4 we use $T_{\text {single }}=8$, but do not place any workahead (buffer size) limitations (Section 4.4). This limitation is instead separately validated in Section 5.5. The focus of the evaluation is on client playback performance (as measured by stall probability and playback rate ${ }^{3}$ and we only consider a single player (possibly competing against other TCP flows, but not other similar players). More extensive multi-factor experiments, experiments with competing players, and more dynamic bandwidth conditions (e.g., as seen by mobile users) provide interesting directions for future work.

\subsubsection{Single-branch experiments}

Consider first a scenario in which the client views a basic interactive branched file (e.g., as shown in Figure 6.1) with a single initial segment $e$ with $n_{e}$ chunks, and a single branch point $b$, with $\left|\mathcal{E}^{b}\right|$ branch options. In our default scenario we let $n_{e}=5$ and $\left|\mathcal{E}^{b}\right|=4$. Furthermore, we use a default end-to-end bandwidth of $2,500 \mathrm{~Kb} / \mathrm{s}$, RTT of $150 \mathrm{~ms}$, and no competing traffic. The use of an RTT of $150 \mathrm{~ms}$ is motivated by typical values observed on our networks. For example, trace route measurements to the top million websites on the Web, according to www.alexa.com on June 12, 2012, suggests that a client within our campus would see an average RTT of $177.4 \mathrm{~ms}$ to these sites.

To evaluate the impact that each of the above experiment parameters has on performance, we use a "one-factor-at-a-time" approach in which we run multiple series of experiments. In each series we vary one parameter at

\footnotetext{
${ }^{3}$ Startup delay is another important metric, but this is small in our experiments and does not differ among the policies.
} 


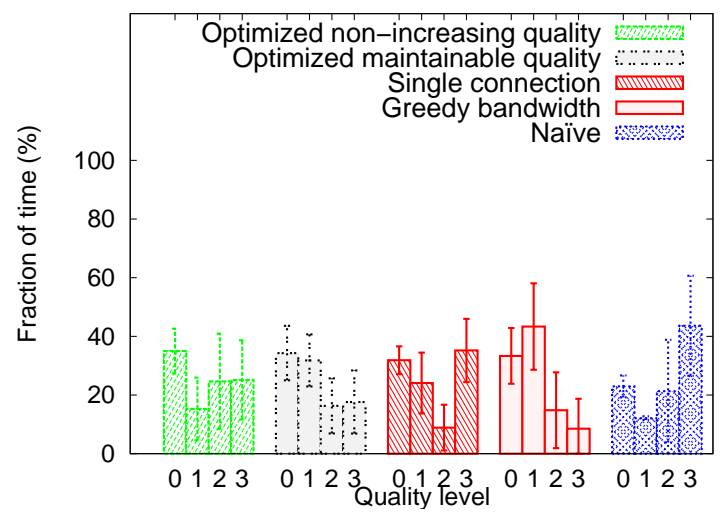

Figure 6.3: Playback qualities in default scenario.

a time, and for each configuration in that series, we perform 30 experiments per policy considered, and report averages and standard deviations for the average video quality and stall probabilities.

In our experiments, we consider a worst-case scenario in which the client always picks the least likely branch, which is the last branch that our prefetching policies prefetch.

Figure 6.3 shows the video quality distribution for our default scenario, based on which we perform our one-factor experiments. Figure 6.4 shows the impact of the available bandwidth on the average video playback rate and stall probability. Here, the data points for $2500 \mathrm{~Kb} / \mathrm{s}$ corresponds to our default scenario, and the average quality simply corresponds to the weighted sum of the quality distributions in Figure 6.3. As expected, the Naïve policy, which does not perform prefetching, always results in the most stall events. Clearly, the high playback rate of this policy is misleading when evaluating the playback quality. All of the prefetching policies successfully employ some of the bandwidth that the Naïve policy uses for downloading the current segment, for prefetching instead, so as to avoid stalls. The most successful tradeoff is achieved by the Optimized maintainable quality policy, which adapts its quality entirely based on our optimization formulation. In contrast to the Optimized non-increasing quality policy, this policy is conservative in that it does not schedule high quality downloads until it expects this quality to be sustainable for the remainder of the segmentbranch pair. The success of a more conservative policy is particularly evident when comparing against the Greedy bandwidth policy, which sees both higher stall probability and lower playback rate than the Optimized maintainable quality policy.

We next take a closer look at the impact of the end-to-end RTTs, number of chunks $n_{e}$ in the initial segment, and the number of branch options $\left|\mathcal{E}^{b}\right|$. Figures 6.5 and 6.6 show the playback rate and stall probability as a 




(a) Playback rate

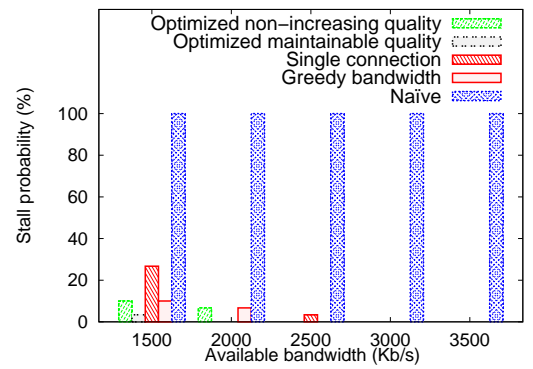

(b) Stall probability

Figure 6.4: Impact of the available bandwidth.

function of these variables. As expected, the average quality observed by the quality-aware policies increase with (i) decreasing RTTs, (ii) increasing number of chunks $n_{e}$ in the initial segment, and (iii) decreasing number of branch options $\left|\mathcal{E}^{b}\right|$. In the case of decreasing RTTs, the TCP throughput quickly ramps up. In the case of increasing number of chunks $n_{e}$, the policies have more time to build a workahead before reaching the branch point, and finally, in the case of decreasing number of branch options, less data must be downloaded ahead of the branch point, leaving bandwidth which instead can be used to download higher quality chunks.

As when comparing across different bandwidth conditions, the qualityaware policies consistently outperform the Greedy bandwidth policy. Under challenging conditions, the Optimized maintainable quality policy is the clear winner, and consistently sees the lowest stall probabilities.

\subsubsection{Multiple branch point scenarios}

In general, we assume that the distance between successive branch points is long enough that it is never worthwhile to prefetch beyond more than a single branch point. We have also performed experiments in which the branched structure has multiple branch points, to assess how the performance differs at the later branch points. In these experiments we have used symmetric structures in which all segments have the same number of chunks $n_{e}$ and all branch points have the same number of branch options $\left|\mathcal{E}^{b}\right|$.

Figures 6.7 and 6.8 show the average playback rate and stall probabilities for the later segments and branch points for experiments with a symmetric tree structure with depth three. While these results are consistent with those observed for the first branch point, we observe slightly higher overall playback rate for these later segments, especially for the prefetching policies with multiple parallel connections. This can be explained by the the fact that these policies can continue to utilize any initiated parallel connections as soon as they have passed an initial branch point. However, in a few cases we also observe higher stall probabilities than for the first branch point. 


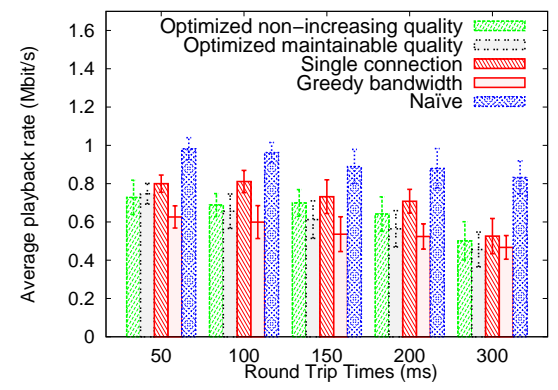

(a) End-to-end RTTs

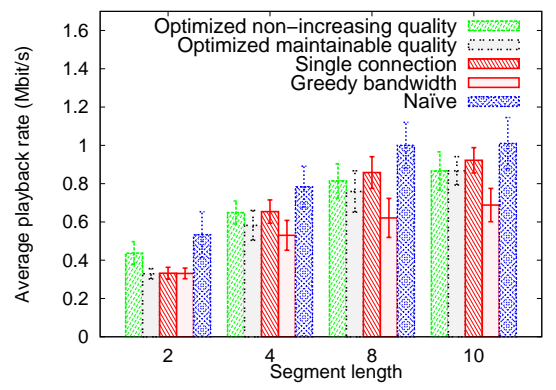

(b) Chunks $n_{e}$

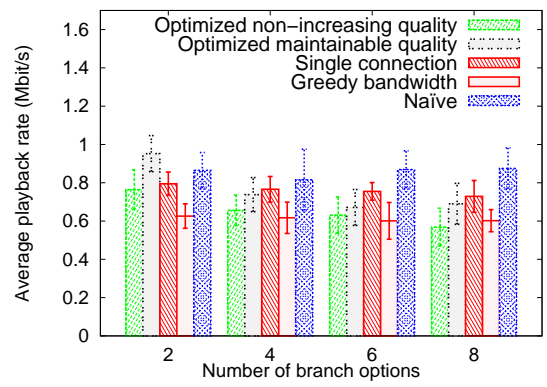

(c) Branch options $\left|\mathcal{E}^{b}\right|$

Figure 6.5: The average playback rate under different end-to-end RTTs, number of chunks $n_{e}$ in the initial segment, and the number of branch options $\left|\mathcal{E}^{b}\right|$.

This is particularly apparent for the case in which we only have two chunks per segment. In this case, segments are too short to allow sufficient time for prefetching chunks from beyond the next branch point, before that branch point is reached.

\subsubsection{Competing flows}

We devise a series of experiments where the available bandwidth is $5 \mathrm{Mb} / \mathrm{s}$, and compare the performance in scenarios with different numbers of competing TCP flows. Each competing flow was generated by downloading a large file from the same server.

As seen from the results shown in Figure 6.9, there is a clear overall performance degradation as the number of competing flows increases. This is to be expected, as in these cases, the fair share of bandwidth available to the player quickly decreases, and the player must adapt the playback quality accordingly. However, in these cases, we can also see significant advantages to our prefetching policies that open multiple parallel connections, compared to the baseline Single connection policy. 


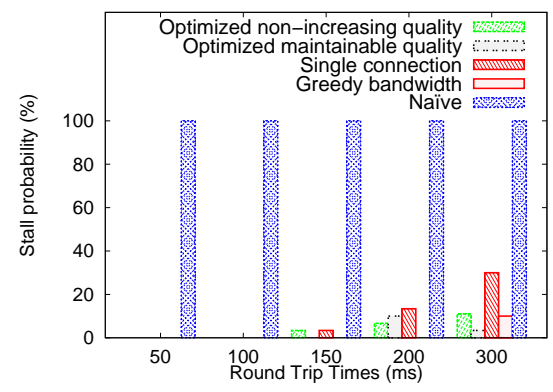

(a) End-to-end RTTs

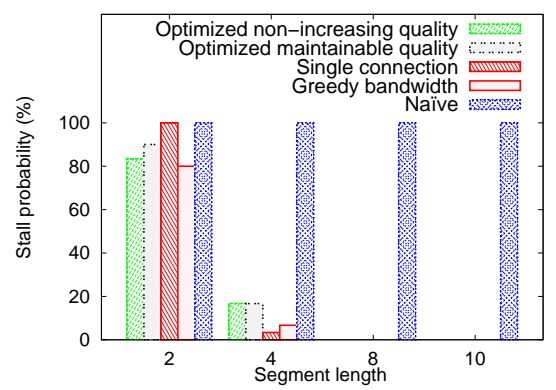

(b) Chunks $n_{e}$

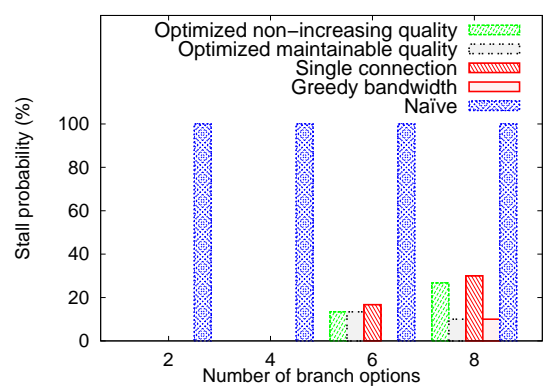

(c) Branch options $\left|\mathcal{E}^{b}\right|$

Figure 6.6: The stall probability under different end-to-end RTTs, number of chunks $n_{e}$ in the initial segment, and the number of branch options $\left|\mathcal{E}^{b}\right|$.

\subsubsection{Capped workahead}

The policies used in Sections 5.2-5.4 do not consider work-ahead (buffer size) limitations. While much of our analysis and that evaluation is focused on seamless playback, we have found that our capped workahead policy (Section 6.3.4) can save substantial bandwidth. To illustrate its operation, we include example results for a high-bandwidth scenario in which a client with $6 \mathrm{Mb} / \mathrm{s}$ connection is playing an interactive branched video with maximum rate encoding at $1.3 \mathrm{Mb} / \mathrm{s}$.

Figure 6.10 shows the variation in the buffer occupancy over time. In this scenario, we use the Optimized maintainable quality policy and the next upcoming branch point has 4 branch alternatives. With our default settings of $T_{\text {single }}=8$ and $\Delta=4$, this gives us $T_{\min }=32$ and $T_{\max }=36$.

It may appear unintuitive that we reach a buffer occupancy much higher than $T_{\max }$. However, this can be explained by the use of many parallel connections. With a high available bandwidth, the player can be fairly aggressive in opening new parallel connections, especially initially when building up the initial buffer to $T_{\max }$. With the buffer only accounting for completed in-order chunk downloads, the buffer occupancy can therefore easily over- 


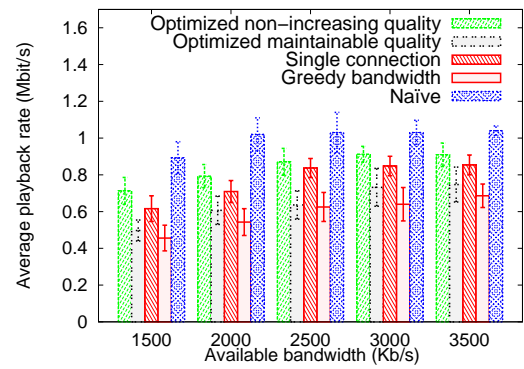

(a) Availible bandwidth

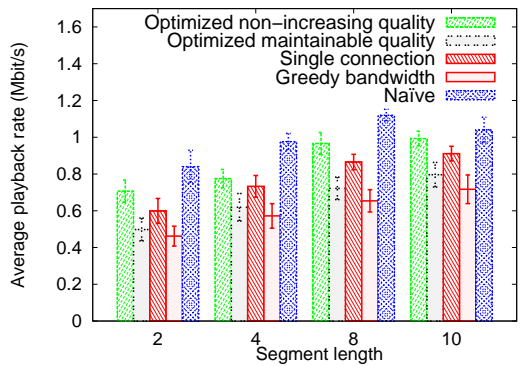

(c) Chunks $n_{e}$



(b) End-to-end RTT

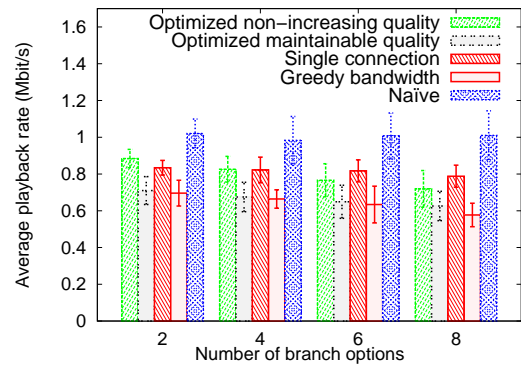

(d) Branch options $\left|\mathcal{E}^{b}\right|$

Figure 6.7: Average playback rate in scenario with multiple branch points, under different conditions.

shoot. However, when reaching (and exceeding) $T_{\max }$ some connections are terminated. With less parallel connections being able to accumulate during the later on periods, as we can see in the figure, the later peaks in buffer occupancy are significantly smaller than the original peak.

\subsection{Conclusions}

Playback interruptions and stall times can greatly impact the users' playback experience. In this chapter we presented the implementation of an interactive branched streaming player that achieves seamless transition between segments in a mutipath structure without playback interruptions. Using a simple optimization framework, we design optimized prefetching policies that maximize the playback quality while ensuring sufficient workahead to avoid stall events. To achieve these goals, our policies (i) adjust the quality levels of the prefetched chunks based on the bandwidth conditions that the client experiences, and (ii) determine the appropriate number of parallel connections to ensure good download speeds and improved workahead buffering. Our results show that our solution is able to achieve rate adaptive streaming and buffer management, such as to ensure seamless playback. 


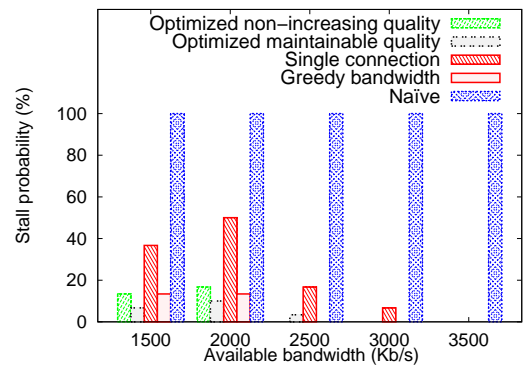

(a) Availible bandwidth

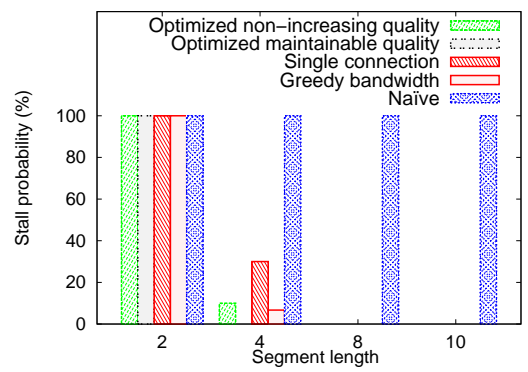

(c) Chunks $n_{e}$

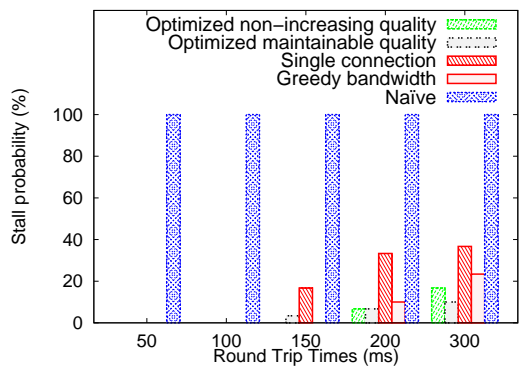

(b) End-to-end RTT



(d) Branch options $\left|\mathcal{E}^{b}\right|$

Figure 6.8: Stall probability at the second branch point under different conditions.

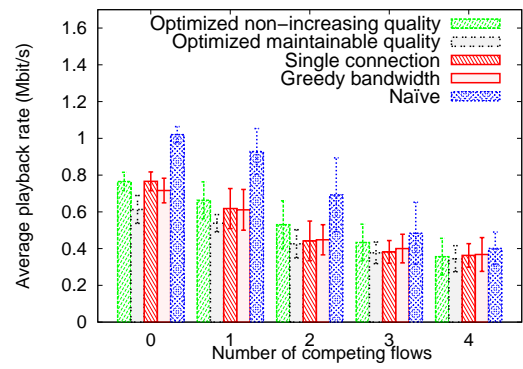

(a) Playback rate

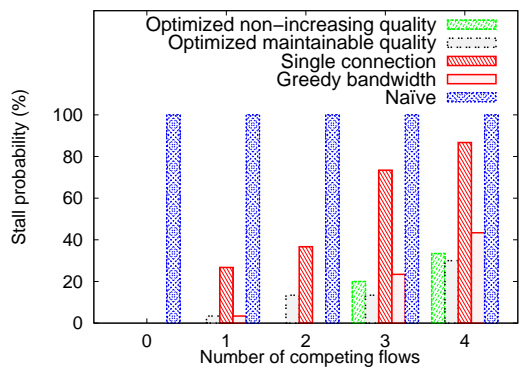

(b) Stall probability

Figure 6.9: The average video playback rate and stall events for different number of competing flows.

We also show that the proposed solution effectively adapts the quality selection and number of parallel connections so as to provide the user with the best possible video quality under scenarios with different bandwidths, RTTs, segment lengths leading to a branch point, number of branch options after a branch point, and number of competing TCP flows. While 


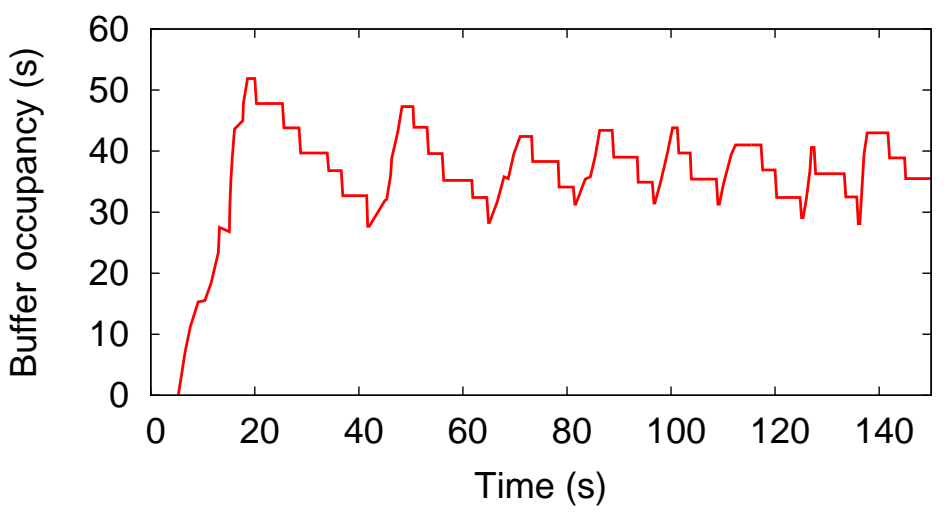

Figure 6.10: Buffer occupancy over time

our current system is entirely client-driven, future work includes server-side optimizations. 


\section{Chapter 7}

\section{Conclusion and future work}

In recent years, on-demand streaming services over the Internet is increasingly becoming the main source of household entertainment. This thesis contributes towards improving the state-of-the-art of today's content delivery techniques by proposing smart content-aware proxies and a novel category of interactive branched video over HAS. We believe that improvements to the infrastructure and personalized content delivery over HAS are important steps towards realizing several forecasts that have been made regarding tomorrow's video delivery.

This thesis investigated several techniques that can be used to improve the efficiency and scalability of on-demand video streaming services. We evaluate the effects of proxy caches on HAS streams, and design and evaluate several content-aware prefetching policies on a proof-of-concept implementation. Our policies are shown to make the process of delivering content more efficient, and, at the same time, improve the viewer's QoE. Our findings reveal that proxy caches do not always offer a helping hand to HAS streams, but that they often require careful configuration so that their benefits are maximized and that potential drawbacks with their interaction with clients are minimized. Through evaluation of our content-aware policies, using real-world representative experiments, we show several areas of improvements, including how to improve and leverage opportunistic prefetching and content-caching opportunities to significantly reduce operating expenses, increase hit rates, and provide clients with a higher average playback quality.

Modern applications and services offered over the Internet are continually adapting their behavior based on user preferences and other user specific customizations. Using the current state-of-the-art techniques, we have proposed, designed, implemented and evaluated, interactive branched video streaming over HTTP. This class of streaming videos allow the viewer to interactively choose one among several alternative storylines at predefined times while watching a video. Our contributions include an optimization 
framework, which can be used to determine when to prefetch chunks, and which parts of the video to prefetch from several potential branches that the client might chose to follow, when selecting their own personalized storyline, as well as the quality level to be selected for each such chunk download. Our framework and policies are designed to handle a wide range of scenarios, including fluctuating bandwidth, frequent branch switches and other variations that one might encounter in the real world. Our client side implementation minimizes playback stalls and maximizes the playback qualities, and makes sure that the action of switching to a different branch does not lead to interruptions that might affect the playback experience of the viewer.

\subsection{Future work}

There are several interesting avenues along which the work in this thesis can be extended. First, several user-centric measurements based on attached web cameras, mics, and other sensors can be used to deduce the viewer's interest or engagement while watching a particular video. This information can then be used to further personalize the content, determine branch-points that will be offered to that particular viewer, or even predict the choices made by the viewer.

Second, we believe that the increased adoption of HAS will continue to drive innovative architectural solutions that aim to provide better user QoE at lower operational and capital expenses. Several proxy-cache-based optimizations can be performed to improve a subset of metrics that the operator is most interested in. In addition to content-aware prefetching proxies proposed in this thesis, proxies can also be built to pre-push certain parts of a video or entire videos to the client. Such videos should be the most likely to be viewed, or ones that currently are being viewed by the client. By pre-pushing parts of the video to the client, the proxy cache can release some of its future resources. Interestingly, future work could also consider how to intelligently pre-push content to a client based on metrics such as user location in mobile networks. If it is well known that certain areas suffer from poor coverage, or due to other factors that are out of control of the operator, pre-pushing content can help avoid a playback stalls when the client is traversing small stretches with poor transmission characteristics.

Finally, our contributions in this thesis can also be applied to streaming of 360 degree videos. We are currently investigating how to best serve stream-bundles that capture multiple simultaneously recorded views or angles. Under such scenarios, videos from constituent cameras of one or more 360 degree camera are streamed together in a bundle. The stream-bundle consists of several views of a certain scene. In this work, we plan to design optimized policies that similar to those presented in this thesis; maximizes the expected playback quality, and minimizes playback stalls; so as to prefetch alternative streams that the client might switch to in the near future. 


\section{Bibliography}

[1] Sandvine Global Internet Phenomena Report- 2h2014- technical report, 2014.

[2] Cisco visual networking index: Forecast and methodology, 2013-2018, 2014.

[3] Cisco visual networking index: The zettabyte era- trends and analysis, 2015.

[4] J. Nielsen. Nielsen's law of Internet bandwidth. http://www . nngroup.com/articles/law-of-bandwidth/, 1998. Accessed: 24Aug-2015.

[5] ISO/IEC 23009-1:2012: Dynamic Adaptive Streaming over HTTP (DASH). Technical report, 2012.

[6] V. Krishnamoorthi, P. Bergström, N. Carlsson, D. Eager, A. Mahanti, and N. Shahmehri. Empowering the creative user: Personalized HTTP-based adaptive streaming of multi-path nonlinear video. In Proc. ACM SIGCOMM FhMN Workshop, 2013.

[7] V. Krishnamoorthi, N. Carlsson, D. Eager, A. Mahanti, and N. Shahmehri. Helping hand or hidden hurdle: Proxy-assisted HTTP-based adaptive streaming performance. In Proc. IEEE MASCOTS, 2013.

[8] V. Krishnamoorthi, N. Carlsson, D. Eager, A. Mahanti, and N. Shahmehri. Quality-adaptive prefetching for interactive branched video using HTTP-based adaptive streaming. In Proc. ACM Multimedia, 2014.

[9] N. Carlsson, D. Eager, V. Krishnamoorthi, T. Polishchuk, and N. Shahmehri. Optimized adaptive streaming of multi-video stream bundles. Under submission. 
[10] V. Krishnamoorthi, N. Carlsson, D. Eager, A. Mahanti, and N. Shahmehri. Bandwidth-aware prefetching for proactive multi-video preloading and improved has performance. In Proc. ACM Multimedia, 2015.

[11] A. Bianco, V. Krishnamoorthi, L. Nanfang, and L. Giraudo. OpenFlow driven ethernet traffic analysis. In Proc. IEEE ICC, 2014.

[12] H. Schulzrinne, A. Rao, and R. Lanphier. Real Time Streaming Protocol (RTSP). 1998. RFC 2326.

[13] H. Parmar and M. Thornburgh. Real-Time Messaging Protocol (RTMP) specification. Adobe specifications, December, 2012.

[14] H. Schulzrinne, S. Casner, R. Frederick, and V. Jacobson. RTP: A transport protocol for real-time applications. 2003. RFC 3550.

[15] F. Dobrian, V. Sekar, A. Awan, I. Stoica, D. Joseph, A. Ganjam, J. Zhan, and H. Zhang. Understanding the impact of video quality on user engagement. In Proc. ACM SIGCOMM, 2011.

[16] L. Chen, Y.P. Zhou, and D.M. Chiu. A study of user behavior in online VoD services. Computer Communications, 2014.

[17] 3GPP TS 26.247 version 11.1.0, 2012.

[18] A. Luotonen and K. Altis. World-wide web proxies. Computer Networks and ISDN Systems, 1994.

[19] A. Chankhunthod, P. B. Danzig, C. Neerdaels, M. E Schwartz, and K. J. Worrel. A hierarchical Internet object cache. In Proc. Usenix Annual Technical Conference, 1996.

[20] D. Povey and J. Harrison. A distributed Internet cache. In Proc. Australian Computer Science Conference, 1997.

[21] N. Carlsson and D. Eager. Ephemeral content popularity at the edge and implications for on-demand caching. IEEE TPDS, to appear.

[22] B. Maggs and R. Sitaraman. Algorithmic nuggets in content delivery. SIGCOMM Comput. Commun. Rev., July 2015.

[23] T. Huang, N. Handigol, B. Heller, N. McKeown, and R. Johari. Confused, timid, and unstable: Picking a video streaming rate is hard. In Proc. ACM IMC, 2012.

[24] S. Akhshabi, L. Anantakrishnan, A. C. Begen, and C. Dovrolis. What happens when HTTP adaptive streaming players compete for bandwidth? In Proc. ACM NOSSDAV, 2012. 
[25] S. Akhshabi, A. C. Begen, and C. Dovrolis. An experimental evaluation of rate-adaptation algorithms in adaptive streaming over HTTP. In Proc. ACM MMSys, 2011.

[26] S. Benno, J. O. Esteban, and I. Rimac. Adaptive streaming: The network HAS to help. Bell Lab. Tech. Journal, 2011.

[27] V. Venkataraman, K. Yoshida, and P. Francis. Chunkyspread: Heterogeneous unstructured tree-based peer-to-peer multicast. In Proc. IEEE International Conference on Network Protocols, 2006.

[28] M. Castro, P. Druschel, A. Kermarrec, A. Nandi, A. Rowstron, and A. Singh. Splitstream: High-bandwidth multicast in cooperative environments. SIGOPS Oper. Syst. Rev., 2003.

[29] A. Mahanti, D. Eager, M. Vernon, and D. Sundaram-Stukel. Scalable on-demand media streaming with packet loss recovery. ACM SIGCOMM Computer Communication Review, 2001.

[30] B. Fenner, M. Handley, H. Holbrook, and I. Kouvelas. Protocol Independent Multicast - Sparse Mode (PIM-SM). 2006. RFC 4601.

[31] H. Schwarz, D. Marpe, and T. Wiegand. Overview of the scalable video coding extension of the H.264/AVC standard. IEEE Trans. on circuits and systems for video technology, 2007.

[32] Y. Wang, Z. Ma, and Y. Ou. Modeling rate and perceptual quality of scalable video as functions of quantization and frame rate and its application in scalable video adaptation. In Proc. International Packet Video Workshop, 2009.

[33] J. Famaey, S. Latre, N. Bouten, W. Van de Meerssche, B. De Vleeschauwer, W. Van Leekwijck, and F. De Turck. On the merits of SVC-based HTTP adaptive streaming. In Proc. IFIP/IEEE Integrated Network Management, 2013.

[34] A. Gerber and R. Doverspike. Traffic types and growth in backbone networks. In Proc. Optical Fiber Communication Conference, 2011.

[35] B. Cohen. Incentives build robustness in BitTorrent. In Proc. Workshop on Economics of Peer-to-Peer systems, 2003.

[36] A. Legout, G. Urvoy-Keller, and P. Michiardi. Understanding BitTorrent: An experimental perspective. Technical report, 2005.

[37] C. Zhang, P. Dhungel, D. Wu, and K.W. Ross. Unraveling the BitTorrent ecosystem. IEEE Trans. on Parallel and Distributed Systems, 2011. 
[38] C. Huang, J. Li, and K. Ross. Peer-assisted VoD: Making Internet video distribution cheap. In Proc. IPTPS, 2007.

[39] G. Dán and N. Carlsson. Dynamic content allocation for cloud-assisted service of periodic workloads. In Proc. IEEE INFOCOM, 2014.

[40] N. Carlsson, G. Dan, D. Eager, and A. Mahanti. Tradeoffs in cloud and peer-assisted content delivery systems. In Proc. IEEE P2P, 2012.

[41] YouTube statistics. https://www.youtube.com/yt/press/ statistics.html. Accessed: 30-Sep-2015.

[42] S. Saroiu, K. Gummadi, R. Dunn, S. Gribble, and H. Levy. An analysis of Internet content delivery systems. SIGOPS Operating Systems Review, 2002.

[43] G. Peng. CDN: content distribution network. Computing Research Repository (CoRR), 2004.

[44] L. Popa, A. Ghodsi, and I. Stoica. HTTP as the narrow waist of the future Internet. In Proc. ACM SIGCOMM Workshop on Hot Topics in Networks, 2010.

[45] A. Vakali and G. Pallis. Content delivery networks: Status and trends. IEEE Internet Computing, 2003.

[46] J. Dilley, B. Maggs, J. Parikh, H. Prokop, R. Sitaraman, and B. Weihl. Globally distributed content delivery. IEEE Internet Computing, 2002.

[47] R. Wang, D. Butnariu, and J. Rexford. Openflow-based server load balancing gone wild. In Proc. USENIX Conference on Hot Topics in Management of Internet, Cloud, and Enterprise Networks and Services, 2011.

[48] N. Carlsson, D. Eager, A. Gopinathan, and Z. Li. Caching and optimized request routing in cloud-based content delivery systems. Performance Evaluation, 2014.

[49] N. Carlsson and D. Eager. Server selection in large-scale video-ondemand systems. ACM Trans. on Multimedia Computing, Communications, and Applications, 2010.

[50] G. Pallis and A. Vakali. Insight and perspectives for content delivery networks. Communications of the ACM, 2006.

[51] Cisco visual networking index: Forecast and methodology, 2015-2020, 2015.

[52] L. Chenghao, I. Bouazizi, and M. Gabbouj. Advanced rate adaption for unicast streaming of scalable video. In Proc. IEEE ICC, 2010. 
[53] J. Liu, B. Li, and Y. Zhang. Adaptive video multicast over the Internet. IEEE Multimedia, 2003.

[54] L.S. Lam, J.Y.B. Lee, S.C. Liew, and W. Wang. A transparent rate adaptation algorithm for streaming video over the Internet. In Proc. Advanced Information Networking and Applications., 2004.

[55] S. Cheung, M. Ammar, and X. Li. On the use of destination set grouping to improve fairness in multicast video distribution. In Proc. IEEE INFOCOM, 1996.

[56] R. Rejaie, M. Handley, and D. Estrin. Quality adaptation for congestion controlled video playback over the Internet. In Proc. $A C M$ SIGCOMM, 1999.

[57] S. McCanne, V. Jacobson, and M. Vetterli. Receiver-driven layered multicast. In Proc. ACM SIGCOMM, 1996.

[58] C. Krasic, K. Li, and J. Walpole. The case for streaming multimedia with TCP. In Interactive Distributed Multimedia Systems, Lecture Notes in Computer Science. Springer Berlin Heidelberg, 2001.

[59] R. Kuschnig, I. Kofler, and H. Hellwagner. Evaluation of HTTP-based request-response streams for Internet video streaming. In Proc. ACM MMSys, 2011.

[60] C. Liu, I. Bouazizi, and M. Gabbouj. Rate adaptation for adaptive HTTP streaming. In Proc. ACM MMSys, 2011.

[61] H. Yu, D. Zheng, B. Zhao, and W. Zheng. Understanding user behavior in large-scale video-on-demand systems. In Proc. ACM SIGOPS/EuroSys, 2006.

[62] L. Zhi, Z. Xiaoqing, J. Gahm, P. Rong, H. Hao, A.C. Begen, and D. Oran. Probe and adapt: Rate adaptation for HTTP video streaming at scale. IEEE Journal on Selected Areas in Communications, 2014.

[63] M. Allman, V. Paxson, and E. Blanton. TCP congestion control. 2009. RFC 2581.

[64] A. Rao, A. Legout, Y. Lim, D. Towsley, C. Barakat, and W. Dabbous. Network characteristics of video streaming traffic. In Proc. ACM CoNEXT, 2011.

[65] R. Fielding, J. Gettys, J. Mogul, H. Frystyk, L. Masinter, P. Leach, and T. Berners-Lee. HyperText Transfer Protocol-HTTP/1.1. 1999. RFC 2616. 
[66] J. Jiang, V. Sekar, and H. Zhang. Improving fairness, efficiency, and stability in HTTP-based adaptive video streaming with FESTIVE. In Proc. ACM CoNEXT, 2012.

[67] L. De Cicco, V. Caldaralo, V. Palmisano, and S. Mascolo. ELASTIC: A client-side controller for dynamic adaptive streaming over HTTP (DASH). In Proc. IEEE Packet Video Workshop, 2013.

[68] X. Yin, A. Jindal, V. Sekar, and B. Sinopoli. A control-theoretic approach for dynamic adaptive video streaming over HTTP. In Proc. ACM SIGCOMM, 2015.

[69] L. De Cicco and S. Mascolo. An adaptive video streaming control system: Modeling, validation, and performance evaluation. IEEE/ACM Trans. on Networking, 2014.

[70] Z. Li, A. Begen, J. Gahm, Y. Shan, B. Osler, and D. Oran. Streaming video over HTTP with consistent quality. In Proc. ACM MMSys, 2014.

[71] S. Akhshabi, L. Anantakrishnan, C. Dovrolis, and A. C. Begen. Serverbased traffic shaping for stabilizing oscillating adaptive streaming players. In Proc. ACM NOSSDAV, 2013.

[72] T.Y. Huang, R. Johari, N. McKeown, M. Trunnell, and M. Watson. A buffer-based approach to rate adaptation: Evidence from a large video streaming service. In Proc. ACM SIGCOMM, 2014.

[73] M. Belshe, R. Peon, and M. Thomson (Ed.). HyperText Transfer Protocol version 2 (HTTP/2). 2015. RFC 7540.

[74] M. Belshe and R. Peon. SPDY protocol. Technical report, 2012. IETF draft-mbelshe-httpbis-spdy-00.

[75] R. Huysegems, J. van der Hooft, T. Bostoen, P. Alface, S. Petrangeli, T. Wauters, and F. De Turck. HTTP/2-based methods to improve the live experience of adaptive streaming. In Proc. ACM Multimedia, 2015.

[76] J. Iyengar and I. Swett. QUIC: A UDP-based secure and reliable transport for HTTP/2. Technical report, 2015. IETF draft-tsvwgquic-protocol-00.

[77] G. Carlucci, L. De Cicco, and S. Mascolo. HTTP over UDP: An experimental investigation of quic. In Proc. ACM SAC, 2015.

[78] X. Liu, F. Dobrian, H. Milner, J. Jiang, V. Sekar, I. Stoica, and H. Zhang. A case for a coordinated Internet video control plane. In Proc. ACM SIGCOMM, 2012. 
[79] G. Cofano, L. De Cicco, and S. Mascolo. A control architecture for massive adaptive video streaming delivery. In Proc. Workshop on Design, Quality and Deployment of Adaptive Video Streaming, 2014.

[80] A. Ganjam, F. Siddiqui, J. Zhan, X. Liu, I. Stoica, J. Jiang, V. Sekar, and H. Zhang. C3: Internet-scale control plane for video quality optimization. In Proc. USENIX NSDI, 2015.

[81] H. Riiser, H. S. Bergsaker, P. Vigmostad, P. Halvorsen, and C. Griwodz. A comparison of quality scheduling in commercial adaptive HTTP streaming solutions on a $3 \mathrm{G}$ network. In Proc. Workshop on Mobile Video, 2012.

[82] T. Lohmar, T. Einarsson, P. Frojdh, F. Gabin, and M. Kampmann. Dynamic adaptive HTTP streaming of live content. In Proc. IEEE WoWMoM, 2011.

[83] T. Kupka, P. Halvorsen, and C. Griwodz. Performance of on-off traffic stemming from live adaptive segmented HTTP video streaming. In Proc. IEEE LCN, 2012.

[84] V. Padmanabhan and J. Mogul. Using predictive prefetching to improve world wide web latency. ACM Computer Communication Review, 1996.

[85] A. Gouta, D. Hong, A. Kermarrec, and Y. Lelouedec. HTTP adaptive streaming in mobile networks: Characteristics and caching opportunities. In Proc. IEEE MASCOTS, 2013.

[86] M. Zink, J. Schmitt, and R. Steinmetz. Layer-encoded video in scalable adaptive streaming. IEEE Trans. on Multimedia, 2005.

[87] S. Chen, H. Wang, X. Zhang, B. Shen, and S. Wee. Segment-based proxy caching for Internet streaming media delivery. IEEE Multimedia, 2005.

[88] R. Rejaie and J. Kangasharju. Mocha: A quality adaptive multimedia proxy cache for Internet streaming. In Proc. ACM NOSSDAV, 2001.

[89] A. Ali-Eldin, M. Kihl, J. Tordsson, and E. Elmroth. Analysis and characterization of a video-on-demand service workload. In Proc. ACM MMSys, 2015.

[90] V. Jacobson, D. Smetters, J. Thornton, M. Plass, N. Briggs, and R. Braynard. Networking named content. In Proc. ACM CONEXT, 2009 .

[91] B. Mobasher, R. Cooley, and J. Srivastava. Automatic personalization based on web usage mining. Communications of the ACM, 2000. 
[92] G. Linden, B. Smith, and J. York. Amazon. com recommendations: Item-to-item collaborative filtering. IEEE Internet Computing, 2003.

[93] B. Rao and L. Minakakis. Evolution of mobile location-based services. Communications of the ACM, 2003.

[94] A. Vapen, N. Carlsson, A. Mahanti, and N. Shahmehri. A look at the third-party identity management landscape. IEEE Internet Computing, 2016.

[95] J. Davidson, B. Liebald, J. Liu, P. Nandy, T. Van Vleet, U. Gargi, S. Gupta, Y. He, M. Lambert, B. Livingston, and D. Sampath. The YouTube video recommendation system. In Proc. ACM RecSys, 2010.

[96] M. J. Pazzani and D. Billsus. Content-based recommendation systems. In The Adaptive Web: Methods and Strategies of Web Personalization (LNCS). Springer Berlin Heidelberg, 2007.

[97] X. Amatriain. Mining large streams of user data for personalized recommendations. ACM SIGKDD Explorations, 2012.

[98] Y. Koren, R. Bell, and C. Volinsky. Matrix factorization techniques for recommender systems. IEEE Computer, 2009.

[99] Q. Huang, B. Chen, J. Wang, and T. Mei. Personalized video recommendation through graph propagation. ACM Trans. on Multimedia Computing, Communications, and Applications, 2014.

[100] B. Mobasher, H. Dai, T. Luo, and M. Nakagawa. Effective personalization based on association rule discovery from web usage data. In Proc. Workshop on Web Information and Data Management, 2001.

[101] K. Oku, S. Nakajima, J. Miyazaki, and S. Uemura. Context-aware svm for context-dependent information recommendation. Proc. IEEE Mobile Data Management, 2006.

[102] H. Kim and Y. Choi. Emosens: Affective entity scoring, a novel service recommendation framework for mobile platform. In Proc. ACM RecSys, 2011.

[103] Y. Zhao, D. L. Eager, and M. K. Vernon. Scalable on-demand streaming of nonlinear media. IEEE/ACM Trans. on Networking, 2007.

[104] N. Carlsson, A. Mahanti, Z. Li, and D. L. Eager. Optimized periodic broadcast of nonlinear media. IEEE Trans. on Multimedia, 2008.

[105] A. Hu. Video-on-demand broadcasting protocols: A comprehensive study. In Proc. IEEE INFOCOM, 2001.

[106] D. Gotz. Scalable and adaptive streaming for non-linear media. In Proc. ACM Multimedia, 2006. 
[107] B. Meixner and J. Hoffmann. Intelligent download and cache management for interactive non-linear video. Multimedia Tools and Applications, 2014.

[108] C. Griwodz, F. Johnsen, S. Rekkedal, and P. Halvorsen. Caching of interactive multiple choice MPEG-4 presentations. In Proc. IEEE Performance Computing and Communications, 2006.

[109] M. Reisslein, F. Hartanto, and K. Ross. Interactive video streaming with proxy servers. Information Sciences, 2002.

[110] A. Sobe, L. Böszörmenyi, and M. Taschwer. Video notation (ViNo): a formalism for describing and evaluating non-sequential multimedia access. International Journal on Advances in Software, 2010.

[111] B. Meixner, K. Matusik, C. Grill, and H. Kosch. Towards an easy to use authoring tool for interactive non-linear video. Multimedia Tools and Applications, 2014.

[112] U. Spierling, S. Weiß, and W. Müller. Towards accessible authoring tools for interactive storytelling. In Proc. Technologies for Interactive Digital Storytelling and Entertainment, 2006.

[113] D. Johansen, P. Halvorsen, H. Johansen, H. Riiser, C. Gurrin, B. Olstad, C. Griwodz, A. Kvalnes, J. Hurley, and T. Kupka. Search-based composition, streaming and playback of video archive content. Multimedia Tools and Applications, 2012.

[114] P. Merkle, A. Smolic, K. Muller, and T. Wiegand. Multi-view video plus depth representation and coding. In Proc. IEEE International Conference on Image Processing, 2007.

[115] P. Merkle, A. Smolic, K. Muller, and T. Wiegand. Efficient prediction structures for multiview video coding. IEEE Trans. Circuits and Systems for Video Technology, Nov 2007.

[116] E. Kurutepe and T. Sikora. Feasibility of multi-view video streaming over p2p networks. In 3DTV Conference: The True Vision-Capture, Transmission and Display of 3D Video, 2008.

[117] J. Lou, H. Cai, and J. Li. A real-time interactive multi-view video system. In Proc. ACM Multimedia, 2005.

[118] E. Kurutepe, M. Civanlar, and A. Tekalp. Client-driven selective streaming of multiview video for interactive 3DTV. IEEE Trans. on Circuits and Systems for Video Technology, Nov 2007.

[119] A. Smolic and P. Kauff. Interactive 3-D video representation and coding technologies. Proc. of the IEEE, Jan 2005. 
[120] A.C. Begen, T. Akgul, and M. Baugher. Watching video over the web: Part 1: Streaming protocols. IEEE Internet Computing, 2011.

[121] C. Müller and C. Timmerer. A VLC media player plugin enabling dynamic adaptive streaming over HTTP. In Proc. ACM Multimedia, 2011.

[122] J. Le Feuvre, C. Concolato, and J. C. Moissinac. GPAC, open source multimedia framework. In Proc. ACM Multimedia, 2007.

[123] L. Rizzo. Dummynet: A simple approach to the evaluation of network protocols. ACM Computer Communication Review, 1997.

[124] M. Levkov. Video encoding and transcoding recommendations for HTTP dynamic streaming on the Adobe ${ }^{\circledR}$ Flash ${ }^{\circledR}$ platform. Technical report, 2010.

[125] A. Mahanti, N. Carlsson, A. Mahanti, M. Arlitt, and C. Williamson. A tale of the tails: Power-laws in Internet measurements. IEEE Network, 2013.

[126] P. Gill, M. Arlitt, Z. Li, and A. Mahanti. YouTube traffic characterization: A view from the edge. In Proc. ACM IMC, 2007.

[127] Jeffrey Erman, Alexandre Gerber, Subhabrata Sen, Oliver Spatscheck, and Kadangode Ramakrishnan. Over the top video: The gorilla in cellular networks. In Proc. ACM IMC, 2011.

[128] Sandvine Global Internet Phenomena Report- 2h2013- technical report, 2013.

[129] TY. Huang, R. Johari, and N. McKeown. Downton Abbey without the hiccups: Buffer-based rate adaptation for HTTP video streaming. In Proc. ACM SIGCOMM Workshop on FhMN, 2013.

[130] E. Altman, D. Barman, B. Tuffin, and M. Vojnovic. Parallel TCP sockets: Simple model, throughput and validation. In Proc. IEEE INFOCOM, 2006. 


\section{Department of Computer and Information Science Linköpings universitet}

\section{Licentiate Thes es}

\section{Linköpings Studies in Science and Technology Faculty of Arts and Sciences}

No 17

No 28

No 29

No 48

No 52

No 60

No 71

No 72

No 73

No 74

No 104

No 108

No 111

No 113

No 118

No 126

No 127

No 139

No 140

No 146

No 150

No 165

No 166

No 174

No 177

No 181

No 184

No 187

No 189

No 196

No 197

No 203

No 212

No 230

No 237

No 250

No 253

No 260

No 283

No 298

No 318

No 319

No 326

No 328

No 333

No 335

No 348

No 352

No 371

No 378
Vojin Plavsic: Interleaved Processing of Non-Numerical Data Stored on a Cyclic Memory. (Available at: FOA, Box 1165, S-581 11 Linköping, Sweden. FOA Report B30062E)

Arne Jönsson, Mikael Patel: An Interactive Flowcharting Technique for Communicating and Realizing Algorithms, 1984.

Johnny Eckerland: Retargeting of an Incremental Code Generator, 1984.

Henrik Nordin: On the Use of Typical Cases for Knowledge-Based Consultation and Teaching, 1985.

Zebo Peng: Steps Towards the Formalization of Designing VLSI Systems, 1985.

Johan Fagerström: Simulation and Evaluation of Architecture based on Asynchronous Processes, 1985.

Jalal Maleki: ICONStraint, A Dependency Directed Constraint Maintenance System, 1987.

Tony Larsson: On the Specification and Veri fication of VLSI Systems, 1986.

Ola Strömfors: A Structure Editor for Documents and Programs, 1986.

Christos Levcopoulos: New Results about the Approximation Behavior of the Greedy Triangulation, 1986.

Shamsul I. Chowdhury: Statistical Expert Systems - a Special Application Area for Knowledge-Bas ed Computer Methodology, 1987.

Rober Bilos: Incremental Scanning and Token-Based Editing, 1987.

Hans Block: SPORT-SORT Sorting Algorithms and Sport Tournaments, 1987.

Ralph Rönnquist: Network and Lattice Based Approaches to the Representation of Knowledge, 1987.

Mariam Kamkar, Nahid Shahmehri: Affect-Chaining in Program Flow Analysis Applied to Queries of Programs, 1987.

Dan Strömberg: Transfer and Distribution of Application Programs, 1987.

Kristian Sandahl: Case Studies in Knowledge Acquisition, Migration and User Acceptance of Expert Systems, 1987.

Christer Bäckström : Reasoning about Interdependent Actions, 1988.

Mats Wirén: On Control Strategies and Incrementality in Unification-Based Chart Parsing, 1988.

Johan Hultman: A Software System for Defining and Controlling Actions in a Mechanical System, 1988.

Tim Hansen: Diagnosing Faults using Knowledge about Malfunctioning Behavior, 1988.

Jonas Löwgren: Supporting Design and Management of Expert System User Interfaces, 1989.

Ola Petersson: On Adaptive Sorting in Sequential and Parallel Models, 1989.

Yngve Larsson: Dynamic Configuration in a Distributed Environment, 1989.

Peter Åberg: Design of a Multiple View Presentation and Interaction Manager, 1989.

Henrik Eriksson: A Study in Domain-Oriented Tool Support for Knowledge Acquisition, 1989.

Ivan Rankin: The Deep Generation of Text in Expert Critiquing Systems, 1989.

Simin Nadjm-Tehrani: Contributions to the Declarative Approach to Debugging Prolog Programs, 1989.

Magnus Merkel: Temporal Information in Natural Language, 1989.

Ulf Nilsson: A Systematic Approach to Abstract Interpretation of Logic Programs, 1989.

Staffan Bonnier: Horn Clause Logic with External Procedures: Towards a Theoretical Framework, 1989.

Christer Hansson: A Prototype System for Logical Reasoning about Time and Action, 1990.

Björn Fjellborg: An Approach to Extraction of Pipeline Structures for VLSI High-Level Synthesis, 1990.

Patrick Doherty: A Three-Valued Approach to Non-Monotonic Reasoning, 1990.

Tomas Sokolnicki: Coaching Partial Plans: An Approach to Knowledge-Based Tutoring, 1990.

Lars Strömberg: Postmortem Debugging of Distributed Systems, 1990.

Torbjörn Näslund: SLDFA-Resolution - Computing Answers for Negative Queries, 1990.

Peter D. Holmes: Using Connectivity Graphs to Support Map-Related Reasoning, 1991.

Olof Johansson: Improving Implementation of Graphical User Interfaces for Object-Oriented Knowledge- Bas es, 1991.

Rolf G Larsson: Aktivitetsbaserad kalkylering i ett nytt ekonomisystem, 1991.

Lena Srömbäck: Studies in Extended Unification-Based Formalism for Linguistic Description: An Algorithm for Feature Structures with Disjunction and a Proposal for Flexible Systems, 1992.

Mikael Pettersson: DML-A Language and System for the Generation of Efficient Compilers from Denotational Specification, 1992.

Andreas Kågedal: Logic Programming with External Procedures: an Implementation, 1992.

Patrick Lambrix : Aspects of Version Management of Composite Objects, 1992.

Xinli Gu:T estability Analysis and Improvement in High-Level Synthesis Systems, 1992.

Torbjörn Näslund: On the Role of Evaluations in Iterative Development of Managerial Support Systems, 1992.

Ulf Cederling: Industrial Soffware Development - a Case Study, 1992.

Magnus Morin: Predictable Cyclic Computations in Autonomous Systems: A Computational Model and Implementation, 1992.

Mehran Noghabai: Evaluation of Strategic Investments in Information Technology, 1993.

Mats Larsson: A Transformational Approach to Formal Digital System Design, 1993. 
No 380

No 381

No 383

No 386

No 398

No 402

No 406

No 414

No 417

No 436

No 437

No 440

FHS 3/94

FHS 4/94

No 441

No 446

No 450

No 451

No 452

No 455

FHS 5/94

No 462

No 463

No 464

No 469

No 473

No 475

No 476

No 478

FHS 7/95

No 482

No 488

No 489

No 497

No 498

No 503

FHS 8/95

FHS 9/95

No 513

No 517

No 518

No 522

No 538

No 545

No 546

FiF-a 1/96

No 549

No 550

No 557

No 558

No 561

No 563
Johan Ringström: Compiler Generation for Parallel Languages from Denotational Specifications, 1993. Michael Jansson: Propagation of Change in an Intelligent Information System, 1993.

Jonni Harrius: An Architecture and a Knowledge Representation Model for Expert Critiquing Systems, 1993. Per Österling: Symbolic Modelling of the Dynamic Environments of Autonomous Agents, 1993.

Johan Boye: Dependency-based Groudness Analysis of Functional Logic Programs, 1993.

Lars Degerstedt: Tabulated Resolution for Well Founded Semantics, 1993.

Anna Moberg: Satellitkontor - en studie av kommunikationsmönster vid arbete på distans, 1993.

Peter Carlsson: Separation av företagsledning och finansiering - fallstudier av företagsledarutköp ur ett agentteoretiskt perspektiv, 1994.

Camilla Sjöström: Revision och lagreglering - ett historiskt perspektiv, 1994.

Cecilia Sjöberg: Voices in Design: Argumentation in Participatory Development, 1994.

Lars Viklund: Contributions to a High-level Programming Environment for a Scientific Computing, 1994.

Peter Loborg: Error Recovery Support in Manufacturing Control Systems, 1994.

Owen Eriksson: Informations
samskapande perspektiv, 1994.

Karin Pettersson: In formationssystemstrukturering, ansvars fördelning och användarin flytande - En komparativ studie med utgångspunkt i två informationssystemstrategier, 1994.

Lars Poignant: Informationsteknologi och företagsetablering - Effekter på produktivitet och region, 1994.

Gustav Fahl: Object Views of Relational Data in Multidatabase Systems, 1994.

Henrik Nilsson: A Declarative Approach to Debugging for Lazy Functional Languages, 1994.

Jonas Lind: Creditor - Firm Relations: an Interdisciplinary Analysis, 1994.

Martin Sköld: Active Rules based on Object Relational Queries - Effi cient Change Monitoring Techniques, 1994.

Pär Carlshamre: A Collaborative Approach to Usability Engineering: Technical Communicators and System Developers in Usability-Oriented Systems Development, 1994.

Stefan Cronholm: Varför CASE-verktyg i systemutveckling? - En motiv- och konsekvensstudie avseende arbetssätt och arbetsformer, 1994.

Mikael Lindvall: A Study of Traceability in Object-Oriented Systems Development, 1994.

Fred rik Nilsson: Strategi och ekonomisk styrning - En studie av Sandviks förvärv av Bahco Verktyg, 1994.

Hans Olsén: Collage Induction: Proving Properties of Logic Programs by Program Synthesis, 1994.

Lars Karlsson: Specification and Synthesis of Plans Using the Features and Fluents Framework, 1995.

Ulf Söderman: On Conceptual Modelling of Mode Switching Systems, 1995.

Choong-ho Yi: Reasoning about Concurrent Actions in theTrajectory Semantics, 1995.

Bo Lagerström: Successiv resultatavräkning av pågående arbeten. - Fallstudier i tre byggföret ag, 1995.

Peter Jonsson: Complexity of State-Variable Planning under Structural Restrictions, 1995.

Anders Avdic: Arbetsintegrerad systemutveckling med kalkylprogram, 1995.

Eva L Ragnemalm: Towards Student Modelling through Collaborative Dialogue with a Learning Companion, 1995.

Eva Toller: Contributions to Parallel Multiparadigm Languages: Combining Object-Oriented and Rule-Based Programming, 1995.

Erik Stoy: A Petri Net Based Unified Representation for Hardware/Software Co-Design, 1995.

Johan Herber: Environment Support for Building Structured Mathematical Models, 1995.

Stefan Svenberg: Structure-Driven Derivation of Inter-Lingual Functor-Argument Trees for Multi-Lingual Generation, 1995.

Hee-Cheol Kim: Prediction and Postdiction under Uncertainty, 1995.

metodanalys, 1995.

Malin Bergvall: Systemförvaltning i praktiken - en kvalitativ studie avseende centrala begrepp, aktiviteter och ansvarsroller, 1995.

Joachim Karlsson: Towards a Strategy for Soffware Requirements Selection, 1995.

Jakob Ax elsson: Schedulability-Driven Partitioning of Heterogeneous Real-Time Systems, 1995.

Göran Forslund: Toward Cooperative Advice-Giving Systems: The Expert Systems Experience, 1995.

Jörgen Andersson: Bilder av småföretag ares ekonomistyrning, 1995.

Staffan Flodin: Efficient Management of Object-Ori ented Queries with Late Binding, 1996.

Vadim Engelson: An Approach to Automatic Construction of Graphical User Interfaces for Applications in Scientific Computing, 1996.

Magnus Werner : Multidatabase Integration using Polymorphic Queries and Views, 1996.

Mikael Lind: Affärsprocessinriktad föränd ringsanalys - utveckling och tillämpning av synsätt och metod, 1996.

Jon as Hallberg: High-Level Synthesis under Local Timing Constraints, 1996.

Kristina Larsen: Förutsättningar och begränsningar för arbete på distans - erfarenh eter från fy ra svenska fö retag. 1996.

Mikael Johansson: Quality Functions for Requirements Engineering Methods, 1996.

Patrik Nordling: The Simulation of Rolling Bearing Dynamics on Parallel Computers, 1996.

Anders Ekman: Exploration of Polygonal Environments, 1996.

Niclas Andersson: Compilation of Mathematical Models to Parallel Code, 1996. 
No 567

No 575

No 576

No 587

No 589

No 591

No 595

No 597

No 598

No 599

No 607

No 609

FiF-a 4

FiF-a 6

No 615

No 623

No 626

No 627

No 629

No 631

No 639

No 640

No 643

No 653

FiF-a 13

No 674

No 676

No 668

No 675

FiF-a 14

No 695

No 700

FiF-a 16

No 712

No 719

No 723

No 725

No 730

No 731

No 733

No 734

FiF-a 21

FiF-a 22

No 737

No 738

FiF-a 25

No 742

No 748

No 751

No 752

No 753

Johan Jenvald: Simulation and Data Collection in Battle Training, 1996.

Niclas Ohlsson: Soffware Quality Engineering by Early Identification of Fault-Prone Modules, 1996.

Mikael Ericsson: Commenting Systems as Design Support-A Wizard-of-Oz Study, 1996.

Jörgen Lindström: Chefers användning av kommunikationsteknik, 1996.

Esa Falkenroth: Data Management in Control Applications - A Proposal Based on Active Database Systems, 1996.

Niclas Wahllöf: A Default Extension to Description Logics and its Applications, 1996.

Annika Larsson: Ekonomisk Styrning och Organisatorisk Passion - ett interaktivt perspektiv, 1997.

Ling Lin: A Value-based Indexing Technique for Time Sequences, 1997.

Rego Granlund: $C^{3}$ Fire - A Microworld Supporting Emergency Management Training, 1997.

Peter Ingels: A Robust T ext Processing Technique Applied to Lexical Error Recovery, 1997.

Per-Arne Persson: Toward a Grounded Theory for Support of Command and Control in Military Coalitions, 1997.

Jonas S Karlsson: A Scalable Data Structure for a Parallel Data Server, 1997.

Carita Åbom: Videomötesteknik i olika affärssituationer - möjligheter och hinder, 1997.

Tommy Wedlund: Att skapa en företags anpassad systemutvecklingsmodell - genom rekonstruktion, värdering och vidareutveckling i T50-bolag inom ABB, 1997.

Silvia Coradeschi: A Decision-Mechanism for Reactive and Coordinated Agents, 1997.

Jan Ollinen: Det flexibla kontorets utveckling på Digital - Ett stöd för multiflex? 1997.

David Byers: Towards Estimating Software Testability Using Static Analysis, 1997.

Fredrik Eklund: Declarative Error Diagnosis of GAPLog Programs, 1997.

Gunilla Ivefors: Krigsspel och Informationsteknik inför en oförutsägbar framtid, 1997.

Jens-Olof Lindh: Analysing Traffic Safety from a Case-Based Reasoning Perspective, 1997

Jukka Mäki-Turja:. Smalltalk - a suitable Real-Time Language, 1997.

Juha Takkinen: CAFE: Towards a Conceptual Model for Information Management in Electronic Mail, 1997.

Man Lin: Formal Analysis of Reactive Rule-based Programs, 1997.

Mats Gustafsson: Bringing Role-Based Access Control to Distributed Systems, 1997.

Boris Karlsson: Metodanalys för förståelse och utveckling av systemutvecklingsverksamhet. Analys och värdering av systemutvecklingsmodeller och dess användning, 1997.

Marcus Bjäreland: Two Aspects of Automating Logics of Action and Change - Regression and Tractability, 1998.

Jan Håkegård: Hierarchical Test Architecture and Board-Level Test Controller Synthesis, 1998.

Per-Ove Zetterlund: Normering av svensk redovisning - En studie av tillkomsten av Redovisningsrådets rekommendation om koncernredovisning (RR01:91), 1998.

Jimmy Tjäder: Projektledaren \& planen - en studie av projektledning i tre installations- och systemutvecklingsprojekt, 1998.

Ulf Melin: Informationssystem vid ökad affärs- och processorientering - egenskaper, strategier och utveckling, 1998.

Tim Heyer: COMPASS: Introduction of Formal Methods in Code Development and Inspection, 1998.

Patrik Hägglund: Programming Languages for Computer Algebra, 1998.

Marie-Theres e Christiansson: Inter-organisatorisk verksamhetsutveckling - metoder som stöd vid utveckling av partnerskap och informationssystem, 1998.

Christina Wennestam: In formation om immateriella resurser. Investeringar i forskning och utveckling samt i personal inom skogsindustrin, 1998.

Joakim Gustafsson: Extending Temporal Action Logic for Ramification and Concurrency, 1998.

Henrik André-Jönsson: Indexing time-series data using text indexing methods, 1999.

Erik Larsson: High-Level Testability Analysis and Enhancement Techniques, 1998.

Carl-Johan Westin: Informationsförsörjning: en fråga om ansvar - aktiviteter och uppdrag i fem stora svenska organisationers operativa informations försörjning, 1998.

Åse Jansson: Miljöhänsyn - en del i företags styrning, 1998.

Thomas Padron-McCarthy: Performance-Polymorphic Declarative Queries, 1998.

Anders Bäckström: Värdeskapande kreditgivning - Kreditriskhantering ur ett agentteoretiskt perspektiv, 1998.

Ulf Seigerroth: Integration av förändringsmetoder - en modell för välgrundad metodintegration, 1999.

Fredrik Öberg: Object-Oriented Frameworks - A New Strategy for Case Tool Development, 1998.

Jonas Mellin: Predictable Event Monitoring, 1998.

Joakim Eriksson: Specifying and Managing Rules in an Active Real-Time Database System, 1998.

Bengt E W Andersson: Samverkande informationssystem mellan aktörer i offentliga åtag anden - En teori om aktörsarenor i samverkan om utbyte av information, 1998.

Pawel Pietrzak: Static Incorrectness Diagnosis of CLP (FD), 1999.

Tobias Ritzau: Real-Time Reference Counting in RT -Java, 1999.

Anders Ferntoft: Elektronisk affärskommunikation - kontaktkostnader och kontaktprocesser mellan kunder och leverantörer på producentmarknader, 1999.

Jo Skåmedal: Arbete på distans och arbetsformens påverk an på resor och resmönster, 1999.

Johan Alvehus: Mötets metaforer. En studie av berättelser om möten, 1999. 
No 754 Magnus Lindahl: Bankens villkor i låneavtal vid kreditgivning till högt belånade företags förvärv: En studie ur ett agentteoretiskt perspektiv, 2000.

No 766 Martin V. Howard: Designing dynamic visualizations of temporal data, 1999.

No 769

No 775

FiF-a 30

No 787

Jesper Andersson: Towards Reactive Software Architectures, 1999.

Anders Henriksson: Unique kernel diagnosis, 1999.

No 788 Håkan Nilsson: Informationsteknik som drivkraft i granskningsprocessen - En studie av fyra revisionsbyråer,

Pär J. Ågerfalk: Pragmatization of Information Systems - A Theoretical and Methodological Outline, 1999.

Charlotte Björkegren: Learning for the next project - Bearers and barriers in knowledge transfer within an organisation, 1999. 2000.

No 790 Erik Berglund: Use-Oriented Documentation in Software Development, 1999.

No 791

No 800

No 807

No 809

FiF-a 32 Klas Gäre: Verksamhets föränd ringar i samband med IS-införande, 1999.

Anders Subotic: Software Quality Inspection, 1999.

Svein Bergum: Managerial communication in telework, 2000

Flavius Gruian: Energy-Aware Design of Digital Systems, 2000.

No 808

No 820

No 823

No 832

FiF-a 34

Karin Hedström: Kunskapsanvändning och kunskapsutveckling hos verksamhetskonsulter - Erfarenheter från ett FOU-samarbete, 2000.

No 842

No 844

FiF-a 37

FiF-a 40

FiF-a 41

No. 854

No 863

No 881

No 882

No 890

FiF-a 47

Linda Askenäs: Affärssystemet - En studie om teknikens aktiva och passiva roll i en organisation, 2000.

Jean Paul Meynard: Control of industrial robots through high-level task programming, 2000.

Lars Hult: Publika Gränsytor - ett designexempel, 2000.

Paul Pop: Scheduling and Communication Synthesis for Distributed Real-Time Systems, 2000.

Göran Hultgren: Nätverksinriktad Förändringsanalys - perspektiv och metoder som stöd för förståelse och utveckling av affärsrelationer och in formationssystem, 2000.

Magnus Kald: The role of management control systems in strategic business units, 2000.

Mikael Cäker: Vad kostar kunden? Modeller för intern redovisning, 2000.

Ewa Braf: Organisationers kunskapsverksamheter - en kritisk studie av "knowledge management", 2000.

Henrik Lindberg: Webbaserade affärsprocesser - Möjligheter och begränsningar, 2000.

Benneth Christiansson: Att komponentbasera informationssystem - Vad säger teori och praktik?, 2000.

Ola Pettersson: Deliberation in a Mobile Robot, 2000.

Dan Lawesson: Towards Behavioral Model Fault Isolation for Object Oriented Control Systems, 2000.

Johan Moe: Execution Tracing of Large Distributed Systems, 2001.

Yuxiao Zhao: XML-based Frameworks for Intern et Commerce and an Implementation of B2B e-procurement, 2001. och aktörssamverkan som förutsättningar för affärsprocesser, 2001.

No 894

No 906

No 917

No 916

Stefan Svarén: Styrning av investeringar i divisionaliserade företag - Ett koncernperspektiv, 2001.

Lin Han: Secure and Scalable E-Service Software Delivery, 2001.

Emma Hansson: Optionsprogram för anställda - en studie av svenska börsföretag, 2001.

Susanne Odar: IT som stöd för strategiska beslut, en studie av datorimplementerade modeller av verksamhet som stöd för beslut om anskaffning av JAS 1982, 2002.

FiF-a-49 Stefan Holgersson: $\Pi$-system och filtrering av verksamhetskunskap - kvalitetsproblem vid analyser och beslutsfattande som bygger på uppgifter hämtade från polisens $\Pi$-system, 2001.

FiF-a-51 Per Oscarsson: In formationssäkerhet $\mathrm{i}$ verksamheter - begrepp och modeller som stöd för förståelse av informationssäkerhet och dess hantering, 2001.

No 919 Luis Alejandro Cortes: A Petri Net Based Modeling and Verification Technique for Real-Time Embedded Systems, 2001.

No 915 Niklas Sandell: Redovisning i skuggan av en bankkris - Värdering av fastigheter. 2001.

No 931 Fredrik Elg: Ett dynamiskt perspektiv på individuella skillnader av heuristisk kompetens, intelligens, mentala modeller, mål och konfidens i kontroll av mikrovärlden Moro, 2002.

No 933 Peter Aronsson: Automatic Parallelization of Simulation Code from Equation Based Simulation Languages, 2002.

No 938

No 942

No 956

Bourhane Kadmiry: Fuzzy Control of Unmanned Helicopter, 2002.

Patrik Haslum: Prediction as a Knowledge Representation Problem: A Case Study in Model Design, 2002.

Robert Sevenius: On the instruments of governance - A law \& economics study of capital instruments in limited liability companies, 2002.

FiF-a 58 Johan Petersson: Lokala elektroniska marknadsplatser - informationssystem för platsbundna affärer, 2002.

No 964

No 973

No 958

FiF-a 61

No 985

No 982

No 989

No 990

Peter Bunus: Debugging and Structural Analysis of Declarative Equation-Based Languages, 2002.

Gert Jervan: High-Level Test Generation and Built-In Self-Test Techniques for Digital Systems, 2002.

Fredrika Berglund: Management Control and Strategy - a Case Study of Pharmaceutical Drug Development, 2002.

Fredrik Karlsson: Meta-Method for Method Configuration - A Rational Unified Process Case, 2002.

Sorin Manolache: Schedulability Analysis of Real-T ime Systems with Stochastic Task Execution Times, 2002.

Diana Szentiványi: Performan ce and Availability Trade-offs in Fault-Tolerant Middleware, 2002.

Iakov Nakhimovski: Modeling and Simulation of Contacting Flexible Bodies in Multibody Systems, 2002.

Levon Saldamli: PDEModelica - Towards a High-Level Language for Modeling with Partial Differential Equations, 2002.

No 991 Almut Herzog: Secure Execution Environment for Java Electronic Services, 2002. 
No 999

No 1000

No 1001

No 988

FiF-a 62

No 1003

No 1005

No 1008

No 1010

No 1015

No 1018

No 1022

FiF-a 65

No 1024

No 1034

No 1033

FiF-a 69

No 1049

No 1052

No 1054

FiF-a 71

No 1055

No 1058

FiF-a 73

No 1079

No 1084

FiF-a 74

No 1094

No 1095

No 1099

No 1110

No 1116

FiF-a 77

No 1126

No 1127

No 1132

No 1130

No 1138

No 1149

No 1156

No 1162

No 1165

FiF-a 84

No 1166

No 1167

No 1168

FiF-a 85

No 1171

FiF-a 86

No 1172

No 1183

No 1184

No 1185

No 1190

Jon Edvardsson: Contributions to Program- and Specification-based T est Data Generation, 2002.

Anders Arpteg: Adaptive Semi-structured Information Extraction, 2002.

Andrzej Bednarski: A Dynamic Programming Approach to Optimal Retargetable Code Generation for Irregular Architectures, 2002.

Mattias Arvola: Good to use! : Use quality of multi-user applications in the home, 2003.

Lennart Ljung: Utveckling av en projektivitetsmodell - om organisationers förmåga att tillämpa projektarbets formen, 2003.

Pernilla Qvarfordt: User experience of spoken feedback in multimodal interaction, 2003.

Alex ander Siemers: Visualization of Dynamic Multibody Simulation With Special Reference to Contacts, 2003.

Jens Gustavsson: Towards Unanticipated Runtime Software Evolution, 2003.

Calin Curescu: Adaptive QoS-aware Resource Allocation for Wireless Networks, 2003.

Anna Andersson: Management Information Systems in Process-oriented Healthcare Organisations, 2003.

Björn Johansson: Feedforward Control in Dynamic Situations, 2003.

Traian Pop: Scheduling and Optimisation of Heterogeneous Time/Event-Triggered Distributed Embedded Systems, 2003.

Britt-Marie Johansson: Kundkommunikation på distans - en studie om kommunikationsmediets betydelse i affärstransaktioner, 2003.

Aleksandra Tešanovic: T owards Aspectual Component-Based Real-Time System Development, 2003.

Arja Vainio-Larsson: Designing for Use in a Future Context - Five Case Studies in Retrospect, 2003.

Peter Nilsson: Svenska bankers redovisningsval vid reservering för befarade kreditförluster - En studie vid införandet av nya redovisningsregler, 2003.

Fredrik Ericsson: Information Technology for Learning and Acquiring of Work Knowledge, 2003.

Marcus Comstedt: Towards Fine-Grained Binary Composition through Link Time Weaving, 2003.

Åsa Hedenskog: Increasing the Automation of Radio Network Control, 2003.

Claudiu Duma: Security and Efficiency Tradeoffs in Multicast Group Key Management, 2003.

Emma Eliason: Effektanalys av IT -systems handlingsutrymme, 2003.

Carl Cederberg: Experiments in Indirect Fault Injection with Open Source and Industrial Software, 2003.

Daniel Karlsson: Towards Formal Veri fication in a Component-based Reuse Methodology, 2003.

Anders Hjalmarsson: Att etablera och vidmakthålla förbättringsverksamhet - behovet av koordination och interaktion vid föränd ring av systemutvecklingsverksamheter, 2004.

Pontus Johansson: Design and Development of Recommender Dialogue Systems, 2004.

Charlotte Stoltz: Calling for Call Centres - A Study of Call Centre Locations in a Swedish Rural Region, 2004.

Björn Johansson: Deciding on Using Application Service Provision in SMEs, 2004.

Genevieve Gorrell: Language Modelling and Error Handling in Spoken Dialogue Systems, 2004.

Ulf Johansson: Rule Extraction - the Key to Accurate and Comprehensible Data Mining Models, 2004.

Sonia Sangari: Computational Models of Some Communicative Head Movements, 2004.

Hans Nässla: Intra-Family Information Flow and Prospects for Communication Systems, 2004.

Henrik Sällberg: On the value of customer loyalty programs - A study of point programs and switching costs, 2004.

Larsson: Designarbete i systemutvecklingsprocess, 2004.

Andreas Borg: Contribution to Management and Validation of Non-Functional Requirements, 2004.

Per-Ola Kristensson: Large Vocabulary Shorthand Writing on Stylus Keyboard, 2004

Pär-Anders Albinsson: Interacting with Command and Control Systems: Tools for Operators and Designers, 2004.

Ioan Chisalita: Safety-Ori ented Communication in Mobile Networks for Vehicles, 2004.

Thomas Gustafsson: Maintaining Data Consistency in Embedded Databases for Vehicular Systems, 2004.

Vaida Jakoniené: A Study in Integrating Multiple Biological Data Sources, 2005.

Abdil Rashid Mohamed: High-Level Techniques for Built-In Self-T est Resources Optimization, 2005.

Adrian Pop: Contributions to Meta-Modeling Tools and Methods, 2005.

Fidel Vascós Palacios: On the information ex change between physicians and social insurance officers in the sick leave process: an Activity Theoretical perspective, 2005.

Jenny Lagsten: Verksamhetsutvecklande utvärdering i informationssystemprojekt, 2005.

2005.

Christina Keller: Virtual Learning Environments in higher education. A study of students' acceptance of educational technology, 2005.

Cécile Åberg: Integration of organizational work flows and the Semantic Web, 2005

Anders Forsman: Standardisering som grund för informationssamverkan och IT -tjänster - En fallstudie baserad på trafikinformationstjänsten RDS-TMC, 2005.

Yu-Hsing Huang: A systemic traffic accident model, 2005.

Jan Olausson: Att modellera uppdrag - grunder för förståelse av processinriktade in formationssystem i transaktionsintensiva verksamheter, 2005.

Petter Ahlström: Affärsstrategier för seniorbostadsmarknaden, 2005.

Mathias Cöster: Beyond $\Pi \mathrm{T}$ and Productivity - How Digitization Transformed the Graphic Industry, 2005.

Åsa Horzella: Beyond $\Pi$ T and Productivity - Effects of Digitized Information Flows in Grocery Distribution, 2005.

Maria Kollberg: Beyond $\Pi$ and Productivity - Effects of Digitized In formation Flows in the Logging Industry, 2005.

David Dinka: Role and Identity - Experience of technology in professional settings, 2005. 
No 1191 Andreas Hansson: Increasing the Storage Capacity of Recursive Auto-associative Memory by Segmenting Data, 2005 .

No 1192 Nicklas Bergfeldt: Towards Detached Communication for Robot Cooperation, 2005.

No 1194 Dennis Maciuszek: Towards Dependable Virtual Companions for Later Li fe, 2005.

No 1204 Beatrice Alenljung: Decision-making in the Requirements Engineering Process: A Human-centered Approach, 2005 .

No 1206 Anders Larsson: System-on-Chip Test Scheduling and Test Infrastructure Design, 2005.

No 1207 John Wilander: Policy and Implementation Assurance for Software Security, 2005.

No 1209 Andreas Käll: Översättningar av en managementmodell - En studie av in förandet av Balanced Scorecard i ett landsting, 2005.

No 1225 He Tan: Aligning and Merging Biomedical Ontologies, 2006.

No 1228 Artur Wilk: Descriptive Types for XML Query Language Xcerpt, 2006.

No 1229 Per Olof Pettersson: Sampling-based Path Planning for an Autonomous Helicopter, 2006.

No $1231 \quad$ Kalle Burbeck: Adaptive Real-time Anomaly Detection for Safeguarding Critical Networks, 2006.

No 1233 Daniela Mihailescu: Implementation Methodology in Action: A Study of an Enterprise Systems Implementation Methodology, 2006.

No 1244 Jörgen Skågeby: Public and Non-public giffing on the Internet, 2006.

No 1248 Karolina Eliasson:The Use of Case-Based Reasoning in a Human-Robot Dialog System, 2006.

No 1263 Misook Park-Westman: Managing Competence Development Programs in a Cross-Cultural Organisation - What are the Barriers and Enablers, 2006.

FiF-a $90 \quad$ Amra Halilovic: Ett praktikperspektiv på hantering av mjukvarukomponenter, 2006.

No 1272 Raquel Flodström: A Framework for the Strategic Management of In formation Technology, 2006.

No 1277 Viacheslav Izosimov: Scheduling and Optimization of Fault-Tolerant Embedded Systems, 2006.

No 1283 Håkan Hasewinkel: A Blueprint for Using Commercial Games off the Shel fin Defence Training, Education and Research Simulations, 2006.

FiF-a 91 Hanna Broberg: Verksamhetsanpassade IT -stöd - Designteori och metod, 2006.

No 1286 Robert Kaminski: Towards an XML Document Restructuring Framework, 2006.

No 1293

No 1302

No 1303

No 1305

Jiri Trnka: Prerequisites for data sharing in emergency management, 2007.

Björn Hägglund: A Framework for Designing Constraint Stores, 2007.

Daniel Andreasson: Slack-Time Aware Dynamic Routing Schemes for On-Chip Networks, 2007.

Magnus Ingmarsson: Modelling User Tasks and Intentions for Service Discovery in Ubiquitous Computing, 2007.

No 1306 Gustaf Svedjemo: Ontology as Conceptual Schema when Modelling Historical Maps for Database Storage, 2007.

No 1307 Gianpaolo Conte: Navigation Functionalities for an Autonomous UAV Helicopter, 2007.

No 1309 Ola Leifler: User-Centric Critiquing in Command and Control: The DKExpert and ComPlan Approaches, 2007.

No 1312 Henrik Svensson: Embodied simulation as off-line representation, 2007.

No 1313 Zhiyuan He: System-on-Chip Test Scheduling with Defect-Probability and Temperature Considerations, 2007.

No 1317 Jonas EImqvist: Components, Safety Interfaces and Compositional Analysis, 2007.

No 1320 Håkan Sundblad: Question Classification in Question Answering Systems, 2007.

No 1323 Magnus Lundqvist: Information Demand and Use: Improving Information Flow within Small-scale Business Contexts, 2007.

No 1329 Martin Magnusson: Deductive Planning and Composite Actions in Temporal Action Logic, 2007.

No 1331 Mikael Asplund: Restoring Consistency after Network Partitions, 2007.

No 1332 Martin Fransson: Towards Individualized Drug Dosage - General Methods and Case Studies, 2007.

No 1333 Karin Camara: A Visual Query Language Served by a Multi-sensor Environment, 2007.

No 1337 David Broman: Safety, Security, and Semantic Aspects of Equation-Based Object-Oriented Languages and Environments, 2007.

No 1339 Mikhail Chalabine: Invasive Interactive Parallelization, 2007.

No 1351 Susanna Nilsson: A Holistic Approach to Usability Evaluations of Mixed Reality Systems, 2008.

No 1353 Shanai Ardi: A Model and Implementation of a Security Plug-in for the Software Life Cycle, 2008.

No 1356

No 1359

No 1361

No 1363

No 1371

No 1373

No 1381

No 1386

No 1387

No 1392

No 1393

Erik Kuiper: Mobility and Routing in a Delay-tolerant Network of Unmanned Aerial Vehicles, 2008.

Jana Rambusch: Situated Play, 2008.

Martin Karresand: Completing the Picture - Fragments and Back Again, 2008.

Per Nyblom: Dynamic Abstraction for Interleaved Task Planning and Execution, 2008.

Fred rik Lantz Terrain Object Recognition and Context Fusion for Decision Support, 2008.

Martin Östlund: Assistance Plus: 3D-mediated Advice-giving on Pharmaceutical Products, 2008.

Håkan Lundvall: Automatic Parallelization using Pipelining for Equation-Based Simulation Languages, 2008.

Mirko Thorstensson: Using Observers for Model Based Data Collection in Distributed Tactical Operations, 2008.

Bahlol Rahimi: Implementation of Health Inform ation Systems, 2008.

Maria Holmqvist: Word Alignment by Re-using Parallel Phrases, 2008.

Mattias Eriksson: Integrated Software Pipelining, 2009.

No 1401 Annika Öhgren: Towards an Ontology Development Methodology for Small and Medium-sized Enterprises, 2009.

No 1410 Rickard Holsmark: Deadlock Free Routing in Mesh Networks on Chip with Regions, 2009.

No 1421 Sara Stymne: Compound Processing for Phrase-Based Statistical Machine Translation, 2009.

No 1427 Tommy Ellqvist: Supporting Scientific Collaboration through Workflows and Provenance, 2009.

No 1450 Fabian Segelström: Visualisations in Service Design, 2010.

No 1459 Min Bao: System Level Techniques for Temperature-Aware Energy Optimization, 2010.

No 1466 Mohammad Saifullah: Exploring Biologically Inspired Interactive Networks for Object Recognition, 2011 
No 1468 Qiang Liu: Dealing with Missing Mappings and Structure in a Network of Ontologies, 2011.

No 1469 Rux andra Pop: Mapping Concurrent Applications to Multiprocessor Systems with Multithreaded Processors and Network on Chip-Based Interconnections, 2011.

No 1476 Per-Magnus Olsson: Positioning Algorithms for Surveillance Using Unmanned Aerial Vehicles, 2011.

No 1481 Anna Vapen: Contributions to Web Authentication for Untrusted Computers, 2011.

No 1485 Loove Broms: Sustainable Interactions: Studies in the Design of Energy Awareness Artefacts, 2011.

FiF-a 101 Johan Blomkvist: Conceptualising Prototypes in Service Design, 2011.

No 1490 Håkan Warnquist: Computer-Assisted Troubleshooting for Effi cient Off-board Diagnosis, 2011.

No 1503 Jakob Rosén: Predictable Real-Time Applications on Multiprocessor Systems-on-Chip, 2011.

No 1504 Usman Dastgeer: Skeleton Programming for Heterogen eous GPU-based Systems, 2011.

No 1506 David Landén: Complex Task Allocation for Delegation: From Theory to Practice, 2011.

No 1507 Kristian Stavåker: Contributions to Parallel Simulation of Equation-Based Models on Graphics Processing Units, 2011.

No 1509 Mariusz W zorek: Selected Aspects of Navigation and Path Planning in Unmanned Aircraft Systems, 2011.

No 1510 Piotr Rudol: Increasing Autonomy of Unmanned Aircraft Systems Through the Use of Imaging Sensors, 2011.

No 1513 Anders Carstensen: The Evolution of the Connector View Concept: Enterprise Models for Interoperability Solutions in the Extended Enterprise, 2011.

No 1523 Jody Foo: Computational Terminology: Exploring Bilingual and Monolingual Term Extraction, 2012.

No 1550 Anders Fröberg: Models and Tools for Distributed User Interface Development, 2012.

No 1558 Dimitar Nikolov: Optimizing Fault Tolerance for Real-Time Systems, 2012.

No 1582 Dennis Andersson: Mission Experience: How to Model and Capture it to Enable Vicarious Learning, 2013.

No 1586 Massimiliano Raciti: Anomaly Detection and its Adaptation: Studies on Cyber-physical Systems, 2013.

No 1588 Banafsheh Khademhosseinieh:Towards an Approach for Efficiency Evaluation of Enterprise Modeling Methods, 2013.

No 1589 Amy Rankin: Resilience in High Risk Work: Analysing Adaptive Performance, 2013.

No 1592 Martin Sjölund: Tools for Understanding, Debugging, and Simulation Performance Improvement of EquationBased Models, 2013.

No 1606 Karl Hammar: Towards an Ontology Design Pattern Quality Model, 2013.

No 1624 Maria Vasilevskaya: Designing Security-enhanced Embedded Systems: Bridging Two Islands of Expertise, 2013.

No 1627 Ekhiotz Verga ra: Exploiting Energy Awareness in Mobile Communication, 2013.

No 1644 Valentina Ivanova: Integration of Ontology Alignment and Ontology Debugging for Taxonomy Networks, 2014.

No 1647 Dag Sonntag: A Study of Chain Graph Interpretations, 2014.

No 1657 Kiril Kiryazov: Grounding Emotion Appraisal in Autonomous Humanoids, 2014.

No 1683 Zatan Dragisic: Completing the Is-a Structure in Description Logics Ontologies, 2014.

No 1688 Erik Hansson: Code Generation and Global Optimization Techniques for a Reconfigurable PRAM-NUMA Multicore Architecture, 2014.

No 1715 Nicolas Melot: Energy-Efficient Computing over Streams with Massively Parallel Architectures, 2015.

No 1716 Mahder Gebremedhin: Automatic and Explicit Parallelization Approaches for Mathematical Simulation Models, 2015.

No 1722 Mikael Nilsson: Effici ent Temporal Reasoning with Uncertainty, 2015.

No 1732

FiF 118

Vladislavs Jahundovics: Automatic Verification of Parameterized Sytems by Over-Approximation, 2015.

No 1758

Camilla Kirkegaard: Adding Challenge to a Teachable Agent in a Virtual Learning Environment, 2016.

Vengatanathan Krishnamoorthi: Efficient and Adaptive Content Delivery of Linear and Interactive Branched Videos, 2016. 